\title{
Environmental Releases for Calendar Year 1993
}

Date Published

July 1994

Prepared for the U.S. Department of Energy Office of Environmental Restoration and Waste Management

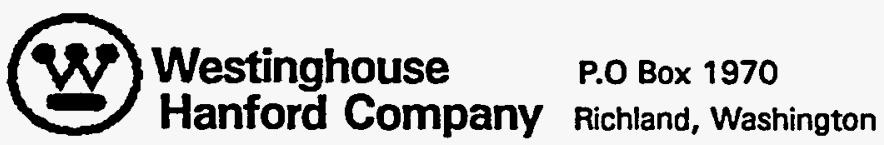

Henford Operations and Engineering Contractor for the

U.S. Department of Energy under Contract DE-AC06-87RL10930

Approved for Public Release 


\section{RELEASE AUTHORIZATION}

Document Number: WHC-EP-0527-3

Document Title: $\quad$ Environmental Releases for Calendar Year 1993

Release Date: $\quad 8 / 17 / 94$

This document was reviewed following the procedures described in WHC-CM-3-4 and is:

APPROVED FOR PUBLIC RELEASE

***************

WHC Information Release Administration Specialist:

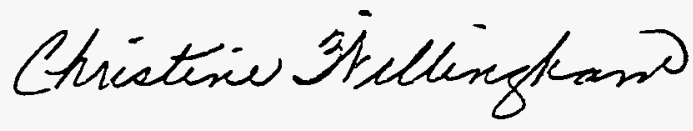

C. Willingham

$8 / 17 / 94$

(Signature)

(Date) 


\section{DISCLAIMER}

This report was prepared as an account of work sponsored by an agency of the United States Government. Neither the United States Government nor any agency thereof, nor any of their employees, make any warranty, express or implied, or assumes any legal liability or responsibility for the accuracy, completeness, or usefulness of any information, apparatus, product, or process disclosed, or represents that its use would not infringe privately owned rights. Reference herein to any specific commercial product, process, or service by trade name, trademark, manufacturer, or otherwise does not necessarily constitute or imply its endorsement, recommendation, or favoring by the United States Government or any agency thereof. The views and opinions of authors expressed herein do not necessarily state or reflect those of the United States Government or any agency thereof. 


\section{DISCLAIMER}

Portions of this document may be illegible in electronic image products. Images are produced from the best available original document. 
Document Title: Environmental Releases for Calendar Year 1993

Prepared by:
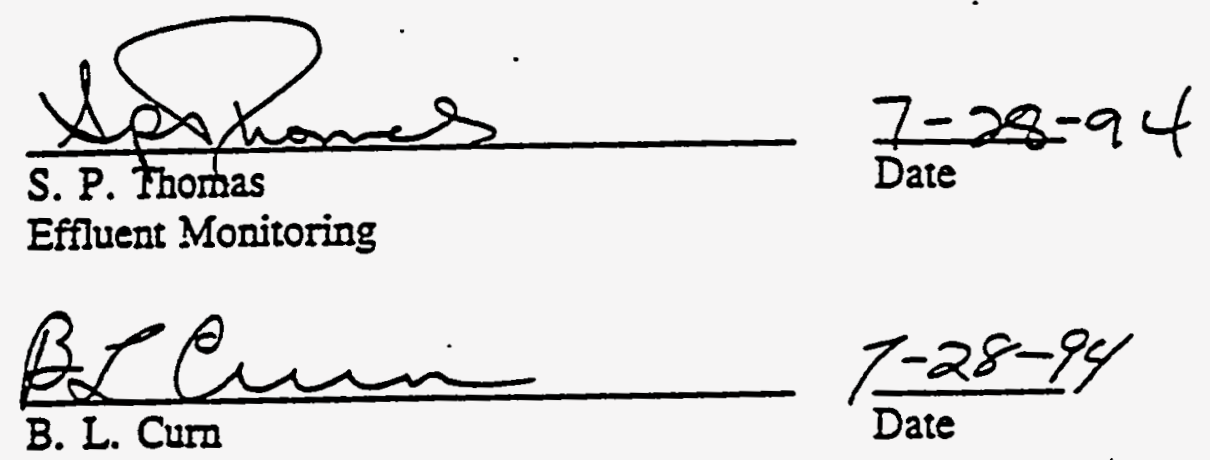
Effluent Monitoring

Approved by:

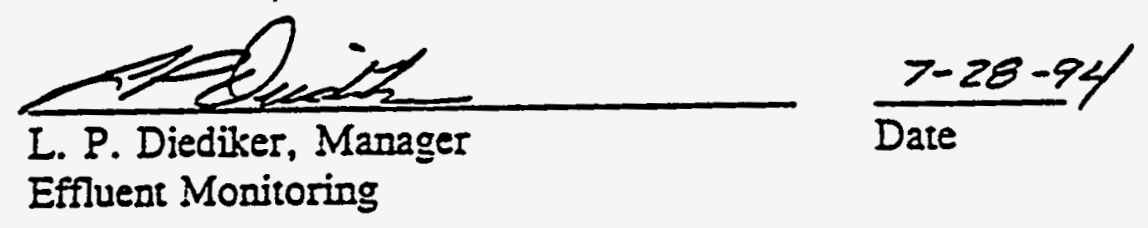


WHC-EP-0527-3

This page intentionally left blank. 
WHC-EP-0527-3

\title{
ENVIRONMENTAL RELEASES FOR
}

CALENDAR YEAR 1993

\begin{abstract}
This report presents data on radioactive and nonradioactive materials released into the environment during calendar year 1993 from facilities managed by Westinghouse Hanford Company. Both summary and detailed presentations of these data are given. When appropriate, comparisons to data from previous years are made.
\end{abstract}


WHC-EP-0527-3

This page intentionally left blank. 
WHC-EP-0527-3

\section{EXECUTIVE SUMMARY}

This report fulfills the annual environmental release reporting requirements of

U.S. Department of Energy (DOE) Orders 5484.1* and 5400.1.** It presents summary and detailed data on air emissions, liquid effluents, and hazardous substances released to the environment during calendar year 1993 from facilities managed by Westinghouse Hanford Company (Westinghouse Hanford).

As part of this executive summary, comprehensive data summaries of air emissions and liquid effluents in 1993 are displayed in Tables ES-1 through ES-5. These tables represent the following:

- . Table ES-1. Radionuclide air emissions data (detailed data on emissions are presented in Section 2.0)

- Table ES-2. Data on radioactive liquid effluents discharged to the soil (detailed data on releases from individual waste streams to soil column disposal sites are presented in Section 3.0)

- Table ES-3. Radionuclides discharged to the Columbia River (detailed data are shown in Section 3.0)

- Table ES-4. Nonradioactive air emissions data (detailed information is given in Section 2.0)

- Table ES-5. Total Volumes and Flow Rates of 200/600 Area Liquid Effluents (detailed data are presented in Section 3.0).

*DOE, 1990, Environmental Protection, Safety, and Health Protection Information Reporting Requirements, DOE Order 5484.1, Change 7, U.S. Department of Energy, Washington, D.C.

**DOE, 1990, General Environmental Protection Program, DOE Order 5400.1, Change 1, U.S. Department of Energy, Washington, D.C. 
Table ES-1. Total Radionuclides in Air Emissions Released

During 1993 from Facilities Managed by Westinghouse Hanford Company

\begin{tabular}{|c|c|}
\hline U. Radronuclide $: 4$ & 4.1 Total (Ci) \\
\hline${ }^{3} \mathrm{H}$ & $2.1 \mathrm{E}+00$ \\
\hline${ }^{60} \mathrm{Co}$ & $5.2 \mathrm{E}-06$ \\
\hline${ }^{90} \mathrm{Sr}$ & $3.3 \mathrm{E}-04$ \\
\hline${ }^{106} \mathrm{Ru}$ & $3.7 \mathrm{E}-04$ \\
\hline${ }^{125} \mathrm{Sb}$ & $6.0 \mathrm{E}-06$ \\
\hline${ }^{129} \mathrm{~T}$ & $4.9 \mathrm{E}-03$ \\
\hline${ }^{134} \mathrm{Cs}$ & $8.9 \mathrm{E}-08$ \\
\hline${ }^{137} \mathrm{Cs}$ & $2.0 \mathrm{E}-03$ \\
\hline${ }^{147} \mathrm{Pm}$ & $1.1 \mathrm{E}-04$ \\
\hline${ }^{154} \mathrm{Eu}$ & $6.3 \mathrm{E}-06$ \\
\hline${ }^{155} \mathrm{Eu}$ & $2.8 \mathrm{E}-06$ \\
\hline${ }^{212} \mathrm{~Pb}$ & $9.7 \mathrm{E}-04$ \\
\hline${ }^{220} \mathrm{Rn}$ & $1.2 \mathrm{E}+01$ \\
\hline $\mathrm{U}$, total & $1.7 \mathrm{E}-05$ \\
\hline${ }^{238} \mathrm{Pu}$ & $4.2 \mathrm{E}-05$ \\
\hline${ }^{239,240} \mathrm{Pu}$ & $5.7 \mathrm{E}-04$ \\
\hline${ }^{241} \mathrm{Pu}$ & $3.5 E-03$ \\
\hline${ }^{241} \mathrm{Am}$ & $1.4 \mathrm{E}-04$ \\
\hline
\end{tabular}


Table ES-2. Total Radionuclides in Liquid Effluents Discharged to Soil During 1993 from Facilities Managed by Westinghouse Hanford Company.

\begin{tabular}{|c|c|}
\hline Radionurliad & Total (ci) \\
\hline${ }^{3} \mathrm{H}$ & $1.5 \mathrm{E}+01$ \\
\hline${ }^{90} \mathrm{Sr}$ & $9.3 \mathrm{E}-02$ \\
\hline${ }^{99} \mathrm{Tc}$ & $4.8 \mathrm{E}-02$ \\
\hline${ }^{137} \mathrm{Cs}$ & $2.2 \mathrm{E}-01$ \\
\hline $\mathrm{U}$, total & $6.9 \mathrm{E}-03$ \\
\hline${ }^{238} \mathrm{Pu}$ & $1.4 \mathrm{E}-03$ \\
\hline${ }^{239,240} \mathrm{Pu}$ & $1.0 \mathrm{E}-01$ \\
\hline${ }^{241} \mathrm{Pu}$ & $2.4 \mathrm{E}-02$ \\
\hline${ }^{241} \mathrm{Am}$ & $1.4 \mathrm{E}-01$ \\
\hline
\end{tabular}

Table ES-3. Total Radionuclides in Liquid Effluents

Discharged to the Columbia River During 1993 from Facilities Managed by Westinghouse Hanford Company.

\begin{tabular}{|c|c|}
\hline Radionuclias & Total (Ci) \\
\hline${ }^{3} \mathrm{H}$ & $3.8 \mathrm{E}-01$ \\
\hline${ }^{60} \mathrm{Co}$ & $3.6 \mathrm{E}-04$ \\
\hline${ }^{90} \mathrm{Sr}$ & $1.1 \mathrm{E}-01$ \\
\hline${ }^{106} \mathrm{Ru}$ & $1.6 \mathrm{E}-03$ \\
\hline${ }^{125} \mathrm{Sb}$ & $1.3 \mathrm{E}-04$ \\
\hline${ }^{134} \mathrm{Cs}$ & $4.7 \mathrm{E}-05$ \\
\hline${ }^{137} \mathrm{Cs}$ & $4.4 \mathrm{E}-04$ \\
\hline${ }^{239,240} \mathrm{Pu}$ & $1.4 \mathrm{E}-07$ \\
\hline
\end{tabular}


Table ES-4. Total Nonradioactive Constituents in Air Emissions Released During 1993 from Facilities Managed by Westinghouse Hanford Company.

\begin{tabular}{|l|c|}
\hline \multicolumn{1}{|c|}{ Constituent } & Quantities (kg) \\
\hline Particulates & 122,000 \\
\hline Sulfur oxides $\left(\mathrm{SO}_{x}\right)$ & 700,000 \\
\hline Nitrogen oxides $\left(\mathrm{NO}_{x}\right)$ & 370,000 \\
\hline Carbon monoxide (CO) & 116,500 \\
\hline Volatile Organic Compounds & 1,390 \\
\hline Lead & 321 \\
\hline
\end{tabular}

Table ES-5. Total Volumes and Flow Rates of Liquid Effluents Discharged to the 200/600 Area Disposal Sites during 1992 and 1993 from Facilities Managed by Westinghouse Hanford Company.

\begin{tabular}{|c|c|c|c|c|c|c|c|}
\hline \multirow{2}{*}{ Surim } & \multirow{2}{*}{ code } & \multirow{2}{*}{ (P) } & \multirow{2}{*}{ Wolponsing } & \multicolumn{2}{|c|}{ \% } & \multicolumn{2}{|c|}{ 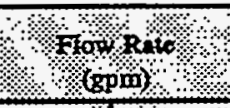 } \\
\hline & & & & $1092 \%$ & 1993. & 1992 & 1993 \\
\hline 207-SL & H101 & 222-S Laboratory chemical sewer & $216-5-26$ crib & $1.7 \mathrm{E}+07$ & $6.5 \mathrm{E}+06$ & 8.4 & 3.3 \\
\hline 207-U & H102 & $\begin{array}{l}\text { UO3 Plant cooling water and steam } \\
\text { condensate }\end{array}$ & 216-U-14 ditch & $2.8 \mathrm{E}+08$ & $2.7 \mathrm{E}+08$ & 140 & 140 \\
\hline $2904-Z A$ & H103 & PFP waste water & $216-Z-20$ crib & $9.0 \mathrm{E}+07$ & $1.0 \mathrm{E}+08$ & 46 & 50 \\
\hline ACW & H108 & 242-A Evaporator cooling water & 216-B-3 pond & $1.8 \mathrm{E}+08$ & $7.0 \mathrm{E}+08$ & 92 & 350 \\
\hline ASC & H110 & $\begin{array}{l}\text { 242-A Evaporator-Crystallizer steam } \\
\text { condensate }\end{array}$ & 216-B-3 pond & $1.3 \mathrm{E}+06$ & $6.7 \mathrm{E}+06$ & 0.65 & 3.4 \\
\hline BCE & $\mathrm{H} 112$ & B Plant chemical sewer & 216-B-63 trench & $5.0 \mathrm{E}+08$ & 0.0 & 250 & 0 \\
\hline $\mathrm{CA} 8$ & H115 & 241-A Tank Farm cooling water & 216-B-3 pond & $7.7 \mathrm{E}+08$ & $8.8 \mathrm{E}+08$ & 390 & 440 \\
\hline CAR & H116 & 244AR Vault cooling water & 216-B-3 pond & $8.9 \mathrm{E}+07$ & $9.0 E+07$ & 45 & 45 \\
\hline $\mathrm{CBC}$ & H117 & B Plant cooling water & 216-B-3 pond & $2.9 E+09$ & $2.5 \mathrm{E}+09$ & 1500 & 1300 \\
\hline CSL & H118 & PUREX chemical scwer & 216-B-3 pond & $5.3 E+08$ & $5.6 \mathrm{E}+08$ & 270 & 280 \\
\hline CWL & H119 & PUREX cooling water & 216-B-3 pond & $6.6 \mathrm{E}+07$ & 0.0 & 65 & 0 \\
\hline LWC & H120 & Laundry waste water & 216-W-LC crib & 2.7E+07 & $2.6 \mathrm{E}+07$ & 14 & 13 \\
\hline U-17 & H126 & UO3 Plant process condensate & 216-U-17 crib & $9.9 \mathrm{E}+05$ & $2.4 \mathrm{E}+06$ & 0.50 & 1.2 \\
\hline
\end{tabular}




\section{CONTENTS}

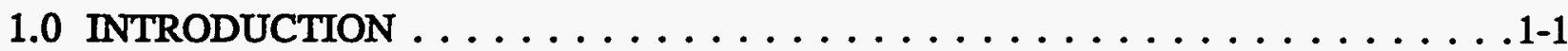

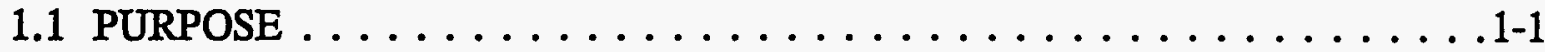

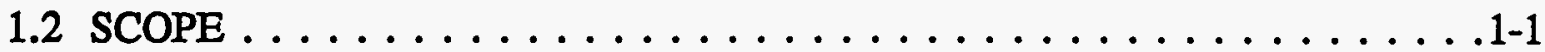

1.3 TYPES AND LOCATIONS OF RELEASES $\ldots \ldots \ldots \ldots \ldots \ldots \ldots \ldots 1-1$

1.4 ENVIRONMENTAL RELEASE LIMITS AND GUIDELINES $\ldots \ldots \ldots 1-2$

1.4.1 Limits for Radioactive Releases . . . . . . . . . . . . 1-2

1.4.2 Limits for Nonradioactive Releases . . . . . . . . . . . . 1-3

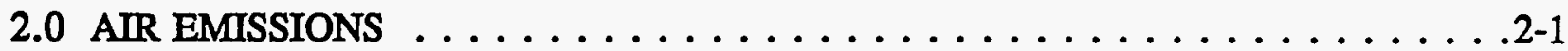

2.1 RADIONUCLIDE AIR EMISSIONS $\ldots \ldots \ldots \ldots \ldots \ldots \ldots \ldots .2-1$

2.1.1 Filtration of Radionuclide Air Emissions . . . . . . . . . . 2-1

2.1.2 Radionuclide Air Emissions . . . . . . . . . . . . . . 2-1

2.1.3 Nonradioactive Constituents in Radionuclide Air Emissions . . . . . 2-2

2.2 NONRADIOACTIVE AIR EMISSIONS $\ldots \ldots \ldots \ldots \ldots \ldots \ldots . \ldots \ldots$

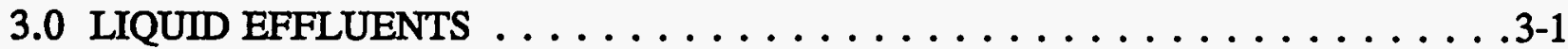

3.1 RADIOACTIVE LIQUID EFFLUENTS $\ldots \ldots \ldots \ldots \ldots \ldots \ldots$. $\ldots \ldots$

3.1.1 Radioactive Constituents in Liquid Effluents . . . . . . . . . . 3-2

3.1.2 Nonradioactive Constituents in Radioactive Liquid Effluents . . . . 3-3

3.2 NONRADIOACTIVE LIQUID EFFLUENTS . . . . . . . . . . . . 3-3

3.2.1 National Pollutant Discharge Elimination System Discharges From the 100 Areas . . . . . . . . . . . . . . . . . 3-3

3.2.2 Sanitary Sewage Discharges to the Soil $\ldots \ldots \ldots \ldots \ldots \ldots$. . . . . .

3.2.3 Process Water Discharges $\ldots \ldots \ldots \ldots \ldots \ldots \ldots . \ldots .3$

4.0 HAZARDOUS SUBSTANCE RELEASES $\ldots \ldots \ldots \ldots \ldots \ldots \ldots \ldots$. . . . . . .

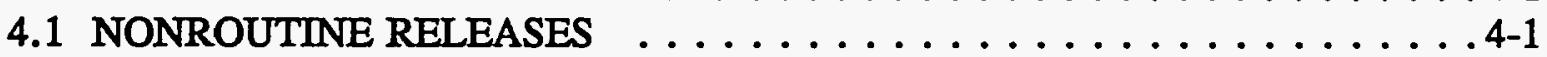

4.2 ROUTINE CONTINUOUS RELEASES $\ldots \ldots \ldots \ldots \ldots \ldots \ldots$. . . . .

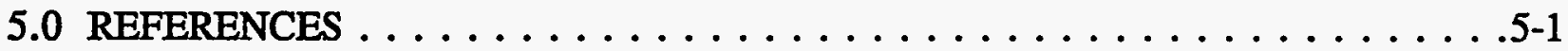

\section{APPENDICES}

A AIR EMISSIONS AND LIQUID EFFLUENTS . . . . . . . . . . . A-1

B MONITORING PROGRAM $\ldots \ldots \ldots \ldots \ldots \ldots \ldots \ldots \ldots$ B-1

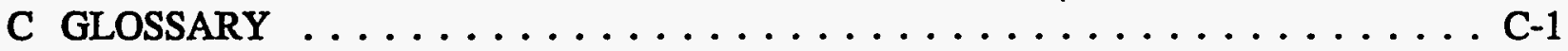


WHC-EP-0527-3

\section{List of Figures}

2-1. Offsite Dose from Airborne Radionuclide Emissions 1990 - 1993 . . . . . . . . 2-2

2-2. Airborne Effluent Releases of ${ }^{239,240} \mathrm{Pu}$ from $1987-1993 \ldots \ldots \ldots$. . . . . . 2-5

2-3. Airborne Effluent Releases of ${ }^{129} \mathrm{I}$ from $1987-1993 \ldots \ldots \ldots$. . . . . 2-5

3-1. Liquid Effluent Releases of ${ }^{90} \mathrm{Sr}$ to the Columbia River from $1987-1993$. . . . . 3-1 
WHC-EP-0527-3

List of Tables

2-1. Radionuclide Air Emissions in 1993 from Facilities, by Area, Managed by Westinghouse Hanford Company . . . . . . . . . . . . 2-4

2-2. Radionuclide Air Emissions from 116-N, 100-N Area . . . . . . . . . . . . . 2-6

2-3. Radionuclide Air Emissions from 105-N 14-Ft Decon Room, 100-N Area . . . . . 2-6

2-4. Radionuclide Air Emissions from 105-N Transfer Area, 100-N Area . . . . . . . 2-7

2-5. Radionuclide Air Emissions from 107-N, 100-N Area . . . . . . . . . . . . 2-7

2-6. Radionuclide Air Emissions from 105-KE, 100-KE Area . . . . . . . . . . . 2-8

2-7. Radionuclide Air Emissions from 105-KW, 100-KW Area . . . . . . . . . . 2-8

2-8. Radionuclide Air Emissions from 1706-KE, 100-KE . . . . . . . . . . . . . . . 2-9

2-9. Radionuclide Air Emissions from 1706-KER, 100-KE Area . . . . . . . . . . 2-9

2-10. Radionuclide Air Emissions from 291-A-1, 200 East Area . . . . . . . . . . . 2-10

2-11. Radionuclide Air Emissions from 296-A-1, 200 East Area . . . . . . . . . . . 2-10

2-12. Radionuclide Air Emissions from 296-A-2, 200 East Area . . . . . . . . . . 2-11

2-13. Radionuclide Air Emissions from 296-A-5A, 200 East Area . . . . . . . . . 2-11

2-14. Radionuclide Air Emissions from 296-A-5B, 200 East Area . . . . . . . . . . 2-11

2-15. Radionuclide Air Emissions from 296-A-6, 200 East Area . . . . . . . . . . . 2-12

2-16. Radionuclide Air Emissions from 296-A-7, 200 East Area . . . . . . . . . . 2-12

2-17. Radionuclide Air Emissions from 296-A-8, 200 East Area . . . . . . . . . . 2-12

2-18. Radionuclide Air Emissions from 296-A-17, 200 East Area . . . . . . . . . 2-13

2-19. Radionuclide Air Emissions from 296-A-21, 200 East Area . . . . . . . . . 2-13

2-20. Radionuclide Air Emissions from 296-A-22, 200 East Area . . . . . . . . 2-13

2-21. Radionuclide Air Emissions from 296-A-27, 200 East Area . . . . . . . . 2-14 
WHC-EP-0527-3

\section{List of Tables (continued)}

2-22. Radionuclide Air Emissions from 296-A-29, 200 East Area . . . . . . . . . . 2-14

2-23. Radionuclide Air Emissions from 296-A-40, 200 East Area . . . . . . . . . 2-14

2-24. Radionuclide Air Emissions from 291-B-1, 200 East Area . . . . . . . . . . . 2-15

2-25. Radionuclide Air Emissions from 296-B-10, 200 East Area . . . . . . . . 2-15

2-26. Radionuclide Air Emissions from 291-S-1, 200 West Area . . . . . . . . . . 2-15

2-27. Radionuclide Air Emissions from 296-S-7W, 200 West Area $\ldots \ldots \ldots$. . . 2-16

2-28. Radionuclide Air Emissions from 296-S-18, 200 West Area . . . . . . . . . 2-16

2-29. Radionuclide Air Emissions from 296-S-21, 200 West Area . . . . . . . . . 2-16

2-30. Radionuclide Air Emissions from 296-W-1, 200 West Area . . . . . . . . . 2-17

2-31. Radionuclide Air Emissions from 291-T-1, 200 West Area . . . . . . . . . 2-17

2-32. Radionuclide Air Emissions from 296-T-11, 200 West Area . . . . . . . . . . 2-17

2-33. Radionuclide Air Emissions from 296-T-12, 200 West Area . . . . . . . . . . 2-18

2-34. Radionuclide Air Emissions from 296-T-13, 200 West Area . . . . . . . . . 2-18

2-35. Radionuclide Air Emissions from 291-U-1, 200 West Area . . . . . . . . 2-18

2-36. Radionuclide Air Emissions from 296-U-2, 200 West Area . . . . . . . . . 2-19

2-37. Radionuclide Air Emissions from 296-U-4, 200 West Area . . . . . . . . . . 2-19

2-38. Radionuclide Air Emissions from 296-U-13, 200 West Area . . . . . . . . . . 2-19

2-39. Radionuclide Air Emissions from 291-Z-1, 200 West Area . . . . . . . . . . 2-20

2-40. Radionuclide Air Emissions from 296-Z-3, 200 West Area . . . . . . . . . 2-20

2-41. Radionuclide Air Emissions from 296-Z-5, 200 West Area . . . . . . . . . . 2-20

2-42. Radionuclide Air Emissions from 308-TRIGA, 300 Area $\ldots . . . \ldots . . .2$ 2-21

2-43. Radionuclide Air Emissions from 340-NT-EX, 300 Area $\ldots . . . \ldots . . .2$ 2-21 
WHC-EP-0527-3

\section{List of Tables (continued)}

2-44. Radionuclide Air Emissions from FFTF-CB-EX, 400 Area . . . . . . . . . 2-21

2-45. Radionuclide Air Emissions from FFTF-RE-SB, 400 Area . . . . . . . . . . 2-21

2-46. Total Alpha and Total Beta Emissions During 1993 from Stacks Managed by

Westinghouse Hanford. . . . . . . . . . . . . . . . . 2-22

2-47. Nonradioactive Constituents in Air Emissions During 1993

from Facilities Managed by Westinghouse Hanford . . . . . . . . . . 2-26

3-1. Radionuclides in Liquid Effluents Discharged to Soil at the Hanford Site in 1993 from Facilities, by Area, Managed by Westinghouse Hanford . . . . . . . . 3-5

3-2. Radionuclide Discharges via N Springs, 100-N Area, to Columbia River . . . . . 3-6

3-3. Radionuclide Discharges via National Pollutant Discharge Elimination System Outfall 009 . . . . . . . . . . . . . . . . . . . . 3-6

3-4. Radionuclide Discharges via National Pollutant Discharge Elimination System

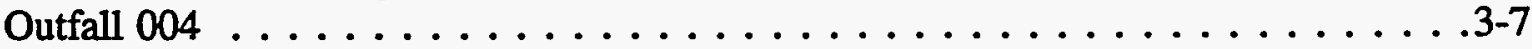

3-5. Radionuclides in 222-S Laboratory Chemical Sewer Discharges (207-SL) to 216-S-26 Crib, 200 West Area . . . . . . . . . . . . . . . 3-7

3-6. Radionuclides in $\mathrm{UO}_{3}$ Plant Discharges (207-U) to 216-U-14 Ditch, 200 West Area . . . . . . . . . . . . . . . . . . . . . . . . 3-8

3-7. Radionuclides in Plutonium Finishing Plant Discharges (2904-ZA) to 216-Z-20 Crib,200 West Area . . . . . . . . . . . . . . . 3-8

3-8. Radionuclides in Discharges from Plutonium-Uranium Extraction Plant 216-A-42 Diversion Basin (A42B3) to 216-B-3 Pond, 200 East Area. . . . . . . . . . . . 3-9

3-9. Radionuclides in 242-A Evaporator Cooling Water Discharges (ACW) to 216-B-3 Pond, 200 East Area . . . . . . . . . . . . . . . . . 3-9

3-10. Radionuclides in 242-A Evaporator-Crystallizer Steam Condensate Discharges (ASC) to 216-B-3 Pond, 200 East Area . . . . . . . . . . . . 3-10

3-11. Radionuclides in 241-A Tank Farm Cooling Water Discharges (CA8) to 216-B-3 Pond, 200 East Area . . . . . . . . . . . . . . . . . . 3-10

3-12. Radionuclides in 244-AR Vault Cooling Water Discharges (CAR) to 216-B-3 Pond, 200 East Area 
WHC-EP-0527-3

List of Tables (continued)

3-13. Radionuclides in B Plant Cooling Water (CBC) Discharges to 216-B-3 Pond, 200 East Area . . . . . . . . . . . . . . . . . . . . . 3-11

3-14. Radionuclides in Plutonium-Uranium Extraction Plant Chemical Sewer

Discharges (CSL) to 216-B-3 Pond, 200 East Area . . . . . . . . . . . 3-12

3-15. Radionuclides in 200 West Area Laundry Discharges (LWC) to 216-W-LC Crib, 200 West Area . . . . . . . . . . . . . . . 3-12

3-16. Radionuclides in Uranium-Oxide Plant Process Condensate Discharges (U-17) to $216-\mathrm{U}-17$ Crib, 200 West Area . . . . . . . . . . . . . . . 3-13

3-17. Radioactivity Discharged via 300 Area Process Sewer System in 1993 . . . . . . 3-13

3-18. National Pollutant Discharge Elimination System Discharge Points . . . . . . 3-14

3-19. Summary of National Pollutant Discharge Elimination System Data . . . . . 3-14

3-20. Sanitary Sewage Discharged, by Area $\ldots \ldots \ldots \ldots \ldots \ldots \ldots$ 3-15 . . . . . .

4-1. Westinghouse Hanford Continuous Comprehensive Environmental Response, Compensation, and Liability Act of 1980 Release Summary for Calendar Year 1993 
WHC-EP-0527-3

\section{LIST OF TERMS}

\begin{tabular}{ll} 
ACW & 242-A Evaporator-Crystallizer cooling water \\
AMU & aqueous makeup \\
ASC & 242-A steam condensate \\
BCE & B Plant Chemical Sewer \\
CAM & continuous air monitor \\
CAR & AR Vault cooling water \\
CBC & B Plant cooling water \\
CERCLA & Comprehensive Environmental Response, Compensation, and Liability Act of 1980 \\
CFR & Code of Federal Regulations \\
CO & carbon monoxide \\
CSL & PUREX Plant chemical sewer line \\
CWL & PUREX Plant cooling water line \\
DCG & derived concentration guide \\
DOE & U.S. Department of Energy \\
DST & double-shell tank \\
Ecology & Washington State Department of Ecology \\
EDE & effective dose equivalent \\
EP & external publication \\
EPA & U.S. Environmental Protection Agency \\
EX & exhaust \\
FFTF & Fast Flux Test Facility \\
GTF & Grout Treatment Facility \\
HEPA & high-efficiency particulate air (filter) \\
HLW & high-level waste \\
KE & 100-K East Area \\
KW & 100-K West Area \\
LLW & low-level waste \\
LWC & laundry waste water crib \\
LWDF & Liquid Waste Disposal Facility \\
MASF & Maintenance and Storage Facility \\
MEI & maximally exposed individual \\
mrem & millirem (unit of exposure) \\
ND & none detected \\
\hline
\end{tabular}


WHC-EP-0527-3

\section{LIST OF TERMS (continued)}

\begin{tabular}{ll}
$\mathrm{NO}_{3}$ & nitrate \\
$\mathrm{NO}_{\mathrm{x}}$ & nitrogen oxides \\
$\mathrm{NPDES}$ & National Pollutant Discharge Elimination System \\
PFP & Plutonium Finishing Plant \\
PSD & Prevention of Significant Deterioration \\
PNL & Pacific Northwest Laboratory \\
POTW & publicly owned treatment works (City of Richland) \\
pPm & parts per million \\
PUREX & Plutonium-Uranium Extraction \\
RCRA & Resource Conservation and Recovery Act of 1976 \\
REDOX & Reduction-Oxidation \\
RL & U.S. Department of Energy, Richland Operations Office \\
RMW & radioactive mixed waste \\
RQ & reportable quantity \\
RWR & raw water return \\
SCD & PUREX coil and steam condensate distillate \\
SO & sulfur oxides \\
SST & single-shell tank \\
TRIGA & Test Reactor and Isotope Production, General Atomics \\
TRU & transuranic (waste) \\
UO & uranium oxide \\
WAC & Washington Administrative Code \\
WESF & Waste Encapsulation Storage Facility \\
Westinghouse & \\
\multicolumn{1}{|c|}{ Hanford } & Westinghouse Hanford Company
\end{tabular}




\section{ENVIRONMENTAL RELEASES FOR \\ CALENDAR YEAR 1993}

\subsection{INTRODUCTION}

Westinghouse Hanford Company (Westinghouse Hanford) is responsible for monitoring radioactive and nonradioactive material released into the environment from facilities it manages for DOE at the Hanford Site. This report documents the environmental releases from those facilities during calendar year 1993. The facilities are located in the 100 Areas (reactor areas), 200 Areas (separations areas), 300 and 400 Areas (research and development areas), 1100 Area (warehouses, stores, and offices), and the 600 Area (all remaining areas of the Hanford Site).

\subsection{PURPOSE}

The purpose of this report is to fulfill the effluent discharge reporting requirements of U.S. Department of Energy (DOE) Orders 5484.1 (DOE 1990) and 5400.1 (DOE 1990a).

\subsection{SCOPE}

This report presents information and data on liquid effluents, air emissions, and nonroutine releases that entered the environment in 1993 from facilities managed by Westinghouse Hanford. When appropriate, comparisons are made to past releases.

\subsection{TYPES AND LOCATIONS OF RELEASES}

Radioactive liquid effluents and air emissions are released from facilities in the 100, 200, 300, and 400 Areas. Radioactive liquid effluents are discharged to the soil in the 200 and 300 Areas and to the Columbia River at the $100-\mathrm{N}$ and $100-\mathrm{K}$ Areas.

The 200 Areas receive radioactive solid wastes for storage and disposal. Radioactive, hazardous solid wastes are also stored in the 200 Areas in a mixed-waste storage facility.

Nonradioactive hazardous solid wastes are stored at interim storage facilities in the 600 Area for eventual transport to an approved offsite treatment, storage, and disposal facility for recycling or disposal.

The major potential sources of nonradioactive air emissions of industrial origin are (1) nitrogenoxide $\left(\mathrm{NO}_{x}\right)$ emissions from the Plutonium-Uranium Extraction (PUREX) and Uranium-Oxide $\left(\mathrm{UO}_{3}\right)$ Plants and (2) fossil-fuel combustion emissions from powerhouse facilities in the 200 and 
300 Areas. During 1993, the PUREX Plant did not operate and therefore did not emit any $\mathrm{NO}_{\mathbf{x}}$. The $\mathrm{UO}_{3}$ Plant emitted 4,843 kg $\left(9,883 \mathrm{lb}\right.$.) of $\mathrm{NO}_{x}$ during a clean-out campaign in 1993.

Waste water from the powerhouse facilities and water treatment facilities in the 200 Areas is discharged to the soil. In the 300 Areas, filter backwash from the water treatment plant is routed to a pond. Sanitary waste water is discharged to (1) the 100-N sanitary sewage lagoon or septic tanks or drain fields in the 100-B, 100-D, 100-H, and 100-K Areas, (2) several septictank or subsurface disposal systems in the 200 Areas, (3) a septic-tank trench system in the 300 Area, and (4) the sewage treatment plant and lagoon in the 400 Area. Solid waste from offices is buried at the Central Landfill in the 600 Area.

\subsection{ENVIRONMENTAL RELEASE LIMITS AND GUIDELINES}

This section presents environmental release standards. Relevant limits and guidelines for nonradioactive constituents also are included in this section. Guidelines are applicable for nonradioactive constituents when they: (1) affect the release and transport of radioactive constituents, (2) are necessary to meet any issued Federal, State, or local permit, or (3) are necessary to meet any Federal, State, or local regulations or guidelines prescribed by the U.S. Department of Energy, Richland Operations Office (RL), or Westinghouse Hanford.

\subsubsection{Limits for Radioactive Releases}

Concentrations of radionuclides in air emissions and liquid effluents from facilities managed by Westinghouse Hanford are regulated by DOE Order 5400.5, Radiation Protection of the Public and the Environment (DOE 1990b) and 40 Code of Federal Regulations (CFR) 61, subpart H (40 CFR 61). The effective dose equivalent (EDE) received by any member of the offsite public from all effluents and emissions released during routine operations at the Hanford Site is not to exceed $100 \mathrm{mrem} / \mathrm{yr}$ from continuous exposure throughout a prolonged period (5 years) and $500 \mathrm{mrem} / \mathrm{yr}$ from noncontinuous, occasional exposure. From the air pathway only, the EDE to any member of the public is not to exceed $10 \mathrm{mrem} / \mathrm{yr}$.

The derived concentration guide (DCG) values in DOE Order 5400.5 apply at the location of actual exposure to members of the public. DCG values are used for comparison purposes only.

The Pacific Northwest Laboratory (PNL), operated for DOE by Battelle Memorial Institute, issues the annual environmental summary report for the Hanford Site (PNL 1994) as required by DOE Order 5400.1 . This report assesses the radiological impact to the public resulting from all Hanford Site operations, in accordance with DOE Order 5480.1B (DOE 1990c) and DOE Order 5400.5. The PNL report uses the release data contained in this report and the Radionuclide Air Emissions Report for the Hanford Site, Calendar Year 1993 (DOE-RL 1994) to calculate the offsite radiological dose impact. The PNL report is used to verify that Westinghouse Hanford is in compliance with the dose standards in DOE Order 5400.5. 
Radioactive solid wastes are managed in accordance with WHC-EP-0063-4, Hanford Site Radioactive Solid Waste Acceptance Criteria (WHC 1993). This manual provides guidelines for managing solid waste to ensure compliance with applicable Federal and State requirements.

Radioactive hazardous solid wastes are managed in accordance with WHC-EP-0063-2 and Chapter 173-303, Washington Administrative Code (WAC), Dangerous Waste Regulations (WAC 173-303).

\subsubsection{Limits for Nonradioactive Releases}

Nitrogen oxides emitted from the PUREX and the $\mathrm{UO}_{3}$ plants are regulated in accordance with the limits defined in Prevention of Significant Deterioration Permit No. PSD-X80-14. All other nonradioactive emissions are monitored for compliance with regulations and guidelines of the Benton-Franklin Counties Clean Air Authority (BFCCAA 1993).

The Resource Conservation and Recovery Act of 1976 (RCRA), the Comprehensive Environmental Response, Compensation, and Liability Act of 1980 (CERCLA), and WAC 173-303 also regulate nonradioactive constituents in air emissions and liquid effluents. Releases and spills of waste materials are reported immediately to appropriate Federal, State, and local agencies when required by CERCLA or WAC 173-303, section 145. Reporting to the National Response Center is required under CERCLA when the released or spilled material exceeded the reportable quantities listed in 40 CFR 302. Reporting to the Washington State Department of Ecology is required when the released or spilled material qualifies as a dangerous waste or extremely dangerous waste and exceeds specified amounts as defined in WAC 173-303, sections 070 to 104 . This report summarizes significant releases and spills reported in 1993 from facilities and areas managed by Westinghouse Hanford.

Westinghouse Hanford manages nonradioactive hazardous solid waste in accordance with WAC 173-303. These wastes are kept at a permit-approved interim storage facility before eventual transport to a recycling center or to an approved offsite disposal facility. Nonradioactive, nonhazardous solid waste is handled in accordance with accepted practices for solid-waste management. 
WHC-EP-0527-3

This page intentionally left blank. 


\subsection{AIR EMISSIONS}

Both radioactive and nonradioactive air emissions are discharged to the atmosphere from facilities managed by Westinghouse Hanford. Release data for each type of emission are discussed separately.

\subsection{RADIONUCLIDE AIR EMISSIONS}

Radionuclide air emissions generally are discharged from stacks and vents. In the 200 Areas, stacks and vents are designated by a number that has a "291" or "296" prefix, depending on their height. Stacks in the 200 Areas that are $61 \mathrm{~m}$ (200 ft) tall are designated by a "291" prefix. All other stacks and vents are designated by the "296" prefix. In the 100, 300, and 400 Areas, stacks and vents usually are identified by facility names.

During 1993, 84 emission points managed by Westinghouse Hanford were registered with the Washington State Department of Health. Information on all of these emissions points (67 of which are in the 200 Areas) and four that are unregistered is included in this report.

\subsubsection{Filtration of Radionuclide Air Emissions}

The following methods are used to remove radionuclides from air emissions:

(1) high-efficiency particulate air (HEPA) filters, (2) sand filters, (3) charcoal absorbers (for iodine removal), (4) water scrubbers, (5) deep-bed fiberglass filters, (6) and fiberglass prefilters. Generally at least one stage, and often several stages, of HEPA filtration is used as the final particulate removal method before the air is discharged to the atmosphere. All in-place HEPA filters are required to have an efficiency of $99.95 \%$ in removing airborne particles larger than $0.3 \mu \mathrm{m}$ in diameter. Filter efficiency is routinely tested. Past release data have shown that radionuclide concentrations in many emissions are below the lower limit of analytical detection. No filtration is provided for those emissions.

\subsubsection{Radionuclide Air Emissions}

Release data on radionuclide air emissions from facilities, by area, managed by Westinghouse Hanford are presented in Table 2-1. Tables 2-2 through 2-45 present data on the radionuclide air emissions from individual stacks and vents managed by Westinghouse Hanford. The data consist of radionuclides detected, average concentrations, and total activities.

Releases of total alpha and total beta radioactivity from all stacks and vents are shown in Table 2-46. Point sources of emissions are sampled only for total alpha and total beta radioactivity when they: (1) cannot potentially contribute $>0.1 \mathrm{mrem} / \mathrm{yr} \mathrm{EDE}$ to the offsite dose, (2) do not emit radionuclides whose total alpha and total beta concentrations exceed the criteria discussed, but (3) have the potential for radionuclide air emissions. 
Radionuclide air emissions from Hanford Site point sources resulted in an offsite dose to the hypothetical maximally exposed individual (MEI) of $0.0063 \mathrm{mrem}$ EDE (see Figure 2-1). This dose is well below the $10 \mathrm{mrem} / \mathrm{yr}$ EDE standard imposed by the U.S. Environmental Protection Agency (EPA) on radionuclide air emissions (40 CFR 61).

Figure 2-1. Offsite Dose from Airborne Radionuclide Emissions 1990 - 1993

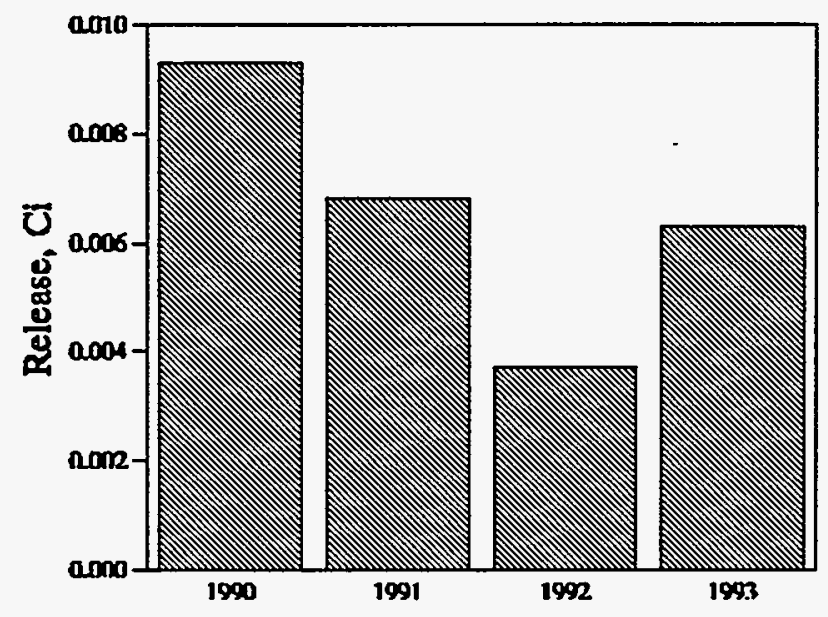

In the 100 Areas, radionuclide emissions at 100-N Area remained below those reported when $\mathrm{N}$ Reactor operated, and the 100-K Area emissions did not change significantly from those of previous years. In the 200 Areas, radionuclide air emissions accounting for the greatest releases were ${ }^{129} \mathrm{I}$ and ${ }^{241} \mathrm{Am}$ via the PUREX Plant main stack and ${ }^{239,240} \mathrm{Pu}$ from PFP, PUREX Plant, and T Plant. Releases from the 300 and 400 Area facilities did not vary substantially from those reported for 1992 (Diediker 1993).

\subsubsection{Nonradioactive Constituents in Radionuclide Air Emissions}

Nitrogen-oxide emissions are released in association with processes at the PUREX and $\mathrm{UO}_{3}$ Plants. PUREX did not operate in 1993; therefore no $\mathrm{NO}_{x}$ emissions were generated or released. $\mathrm{UO}_{3}$ emitted 4,483 kg. $\left(9,883 \mathrm{lb}\right.$.) of $\mathrm{NO}_{x}$ in 1993 . Release limits for $\mathrm{NO}_{x}$ were established by Prevention of Significant Deterioration Permit No. PSD-X80-14, issued by the EPA.

\subsection{NONRADIOACTIVE AIR EMISSIONS}

In 1993, nonradioactive air emissions were discharged from the following Westinghouse Hanford-managed facilities: (1) 200 East (284-E) powerhouse, (2) 200 West (284-W) powerhouse, and (3) the 300 Area powerhouse. Data on emissions from these sources are shown in Table 2-47. Powerhouse stack emissions were calculated using formulas established by the EPA (EPA 1990). 
Fabric-filter collection systems, called baghouses, remove particulate matter and, to a small degree, sulfur oxides $\left(\mathrm{SO}_{x}\right)$, emitted from coal-fired boilers. Baghouses are installed at the coalfired boilers at Westinghouse Hanford-managed facilities in the 200 Areas (284-E and 284-W) and the 300 Area (384) Powerhouse. Anytime a baghouse is bypassed (usually because of unavoidable replacement, repair, and maintenance of equipment), RL and the local air pollution control authority are notified immediately. In 1993, a four day scheduled bypass of the 284-E baghouse occurred. This bypass was pre-approved by the local air pollution control authority. 
Table 2-1. Radionuclide Air Emissions in 1993 from Facilities, by Area, Managed by Westinghouse Hanford Company.

\begin{tabular}{|c|c|c|c|c|c|}
\hline Radioniuclide & $100 \%$ rireas & 200 \%reas & 300 srea & $(100 \%$ rrea & (4.). \\
\hline${ }^{3} \mathrm{H}$ & 2 & - & - & $2.1 \mathrm{E}+00$ & $2.1 \mathrm{E}+00$ \\
\hline${ }^{60} \mathrm{Co}$ & $5.2 \mathrm{E}-06$ & - & - & -- & $5.2 \mathrm{E}-06$ \\
\hline${ }^{90} \mathrm{Sr}$ & $5.4 \mathrm{E}-05$ & $2.7 \mathrm{E}-04^{\mathrm{b}}$ & $2.4 \mathrm{E}-06^{\mathrm{b}}$ & & $3.3 \mathrm{E}-04$ \\
\hline${ }^{106} \mathrm{Ru}$ & $1.3 \mathrm{E}-05$ & $3.6 \mathrm{E}-04$ & - & - & $3.7 \mathrm{E}-04$ \\
\hline${ }^{125} \mathrm{Sb}$ & $6.0 \mathrm{E}-06$ & - & - & - & $6.0 \mathrm{E}-06$ \\
\hline${ }^{129} \mathrm{~T}$ & - & $4.9 \mathrm{E}-03$ & - & - & $4.9 \mathrm{E}-03$ \\
\hline${ }^{134} \mathrm{Cs}$ & $8.9 \mathrm{E}-08$ & & - & - & $8.9 \mathrm{E}-08$ \\
\hline${ }^{137} \mathrm{Cs}$ & $1.6 \mathrm{E}-04$ & $1.8 \mathrm{E}-03$ & - & $8.2 \mathrm{E}-06^{\mathrm{g}}$ & $2.0 \mathrm{E}-03$ \\
\hline${ }^{147} \mathrm{Pm}$ & - & $1.1 \mathrm{E}-04$ & - & -- & $1.1 \mathrm{E}-04$ \\
\hline${ }^{154} \mathrm{Eu}$ & $6.3 \mathrm{E}-06$ & - & - & - & $6.3 E-06$ \\
\hline${ }^{155} \mathrm{Eu}$ & $2.8 \mathrm{E}-06$ & - & - & -- & $2.8 \mathrm{E}-06$ \\
\hline${ }^{212} \mathrm{~Pb}$ & - & $9.7 E-04$ & - & - & $9.7 \mathrm{E}-0.4$ \\
\hline${ }^{220} \mathrm{Rn}$ & - & $1.2 \mathrm{E}+01^{\mathrm{c}}$ & - & - & $1.2 \mathrm{E}+01$ \\
\hline $\mathrm{U}$, total & - & $1.7 E-05$ & $3.6 \mathrm{E}-08^{d}$ & - & $1.7 \mathrm{E}-05$ \\
\hline${ }^{238} \mathrm{Pu}$ & $1.0 \mathrm{E}-06$ & $3.2 \mathrm{E}-06$ & - & - & $4.2 \mathrm{E}-05$ \\
\hline${ }^{239,240} \mathrm{Pu}$ & $8.2 \mathrm{E}-06$ & $5.6 \mathrm{E}-04^{\mathrm{c}}$ & $6.0 \mathrm{E}-07^{\mathrm{f}}$ & $2.4 \mathrm{E}-06$ & $5.7 E-04$ \\
\hline${ }^{241} \mathrm{Pu}$ & - & $3.5 \mathrm{E}-03$ & - & - & $3.5 E-03$ \\
\hline${ }^{241} \mathrm{Am}$ & $5.4 \mathrm{E}-06$ & $1.3 \mathrm{E}-04$ & - & - & $1.4 \mathrm{E}-0.4$ \\
\hline
\end{tabular}

- This symbol indicates sampling was not done for this radionuclide based on its known absence or extremely low concentrations and dose impact.

b This value represents both ${ }^{90} \mathrm{Sr}$ and total beta data. For dose calculations, the total beta data are construed conservatively as ${ }^{90} \mathrm{Sr}$ for those stacks whose emissions are not analyzed for this radionuclide.

c The value of $12 \mathrm{Ci}$ of ${ }^{200} \mathrm{Rn}$ is the result of calculations derived from the measured ${ }^{212} \mathrm{~Pb}$ analyses.

d This value represents total alpha activity in emissions from 306E-ULAB.

- This value represents both total alpha and ${ }^{239,200} \mathrm{Pu}$ emission data. For dose calculations, the total alpha data are construed conservatively as ${ }^{239,200} \mathrm{Pu}$ for those stacks whose emissions are not analyzed for these radionuclides.

${ }^{\mathrm{I}}$ This value represents total alpha activity in emissions from all 300 Area facilities except 306E-ULAB.

2 This value represents both ${ }^{137} \mathrm{Cs}$ and total beta emission data. 


\section{WHC-EP-0527-3}

Figure 2-2. Airborne Effluent Releases of ${ }^{239,240} \mathrm{Pu}$ from $1987-1993$

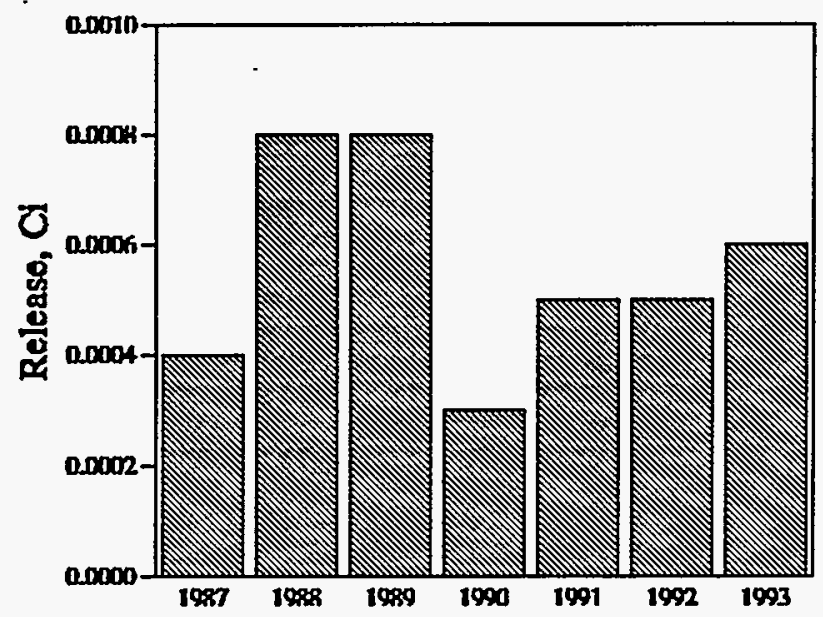

Figure 2-3. Airborne Effluent Releases of ${ }^{129} \mathrm{I}$ from 1987 - 1993

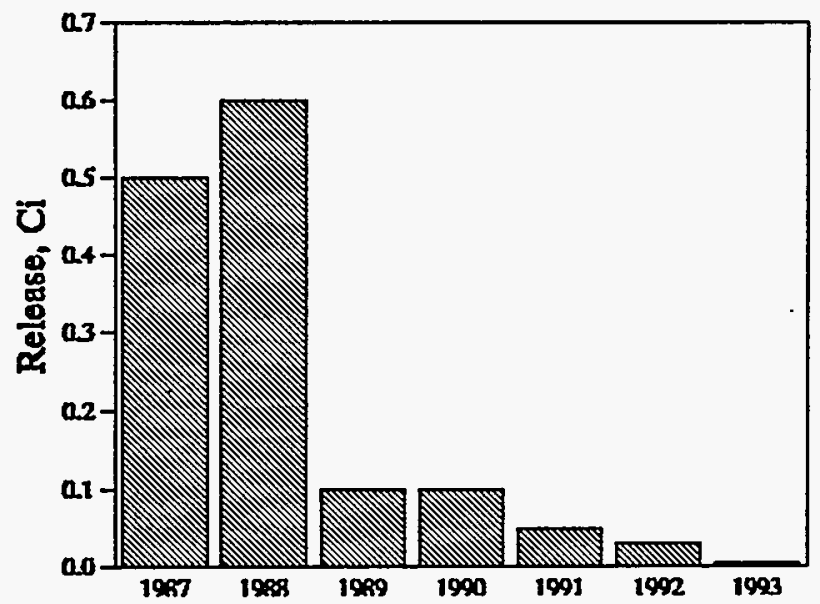


Table 2-2. Radionuclide Air Emissions from 116-N, 100-N Area, in 1993. (Y211)

\begin{tabular}{|c|c|c|}
\hline Radionuclide & 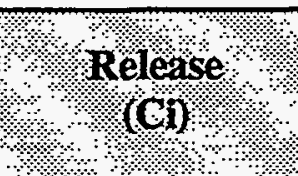 & $\begin{array}{l}\text { Average } \\
\text { Concentration } \\
\text { (wCinil) }\end{array}$ \\
\hline${ }^{60} \mathrm{Co}$ & $2.7 \mathrm{E}-06$ & $2.6 \mathrm{E}-15$ \\
\hline${ }^{90} \mathrm{Sr}$ & $2.4 \mathrm{E}-06$ & $2.3 \mathrm{E}-15$ \\
\hline$\overline{125} \mathrm{Sb}$ & $3.2 \mathrm{E}-06$ & $3.1 \mathrm{E}-15$ \\
\hline $155 \mathrm{Eu}$ & $1.3 \mathrm{E}-06$ & $1.3 \mathrm{E}-15$ \\
\hline${ }^{238} \mathrm{Pu}$ & $2.7 \mathrm{E}-08$ & $2.6 \mathrm{E}-17$ \\
\hline${ }^{239,240} \mathrm{Pu}$ & $4.5 \mathrm{E}-07$ & $4.4 \mathrm{E}-16$ \\
\hline${ }^{241} \mathrm{Am}$ & $1.6 \mathrm{E}-07$ & $1.6 \mathrm{E}-16$ \\
\hline \multicolumn{2}{|l|}{ Total flow (L) } & $1.0 \mathrm{E}+12$ \\
\hline
\end{tabular}

Table 2-3. Radionuclide Air Emissions from 105-N 14-Ft Decon Room, 100-N Area, in 1993. (Y259)

\begin{tabular}{|c|c|c|}
\hline Radionuclide & $\begin{array}{l}\text { Rëlease } \\
(\mathbf{O})\end{array}$ & $\begin{array}{l}\text { A Ayerage } \\
\text { Concentration } \\
(j \mathrm{climL})\end{array}$ \\
\hline${ }^{60} \mathrm{Co}$ & $5.3 \mathrm{E}-08$ & $5.6 \mathrm{E}-16$ \\
\hline${ }^{90} \mathrm{Sr}$ & $6.9 \mathrm{E}-08$ & $7.2 \mathrm{E}-16$ \\
\hline${ }^{137} \mathrm{Cs}$ & $6.9 E-08$ & $7.3 \mathrm{E}-16$ \\
\hline${ }^{154} \mathrm{Eu}$ & $4.3 \mathrm{E}-07$ & $4.5 \mathrm{E}-15$ \\
\hline${ }^{239,240} \mathrm{Pu}$ & $5.1 E-10$ & $5.4 E-18$ \\
\hline${ }^{241} \mathrm{Am}$ & $2.1 \mathrm{E}-09$ & $2.2 \mathrm{E}-17$ \\
\hline \multicolumn{2}{|l|}{ Total flow (L) } & $9.5 \mathrm{E}+10$ \\
\hline
\end{tabular}


Table 2-4. Radionuclide Air Emissions from 105-N Transfer Area, 100-N Area, in 1993. (Y261)

\begin{tabular}{|c|c|c|}
\hline Radionuclide & Selease & $\begin{array}{l}\text { Concentration } \\
\text { (preinit) }\end{array}$ \\
\hline${ }^{60} \mathrm{Co}$ & $2.3 \mathrm{E}-07$ & $7.4 E-16$ \\
\hline${ }^{90} \mathrm{Sr}$ & $1.0 \mathrm{E}-07$ & $3.2 \mathrm{E}-16$ \\
\hline${ }^{106} \mathrm{Ru}$ & $1.8 \mathrm{E}-06$ & $5.8 \mathrm{E}-15$ \\
\hline${ }^{125} \mathrm{Sb}$ & $5.4 \mathrm{E}-07$ & $1.7 \mathrm{E}-15$ \\
\hline${ }^{155} \mathrm{Eu}$ & $3.3 \mathrm{E}-07$ & $1.1 \mathrm{E}-15$ \\
\hline${ }^{239,240} \mathrm{Pu}$ & $1.4 \mathrm{E}-09$ & $4.6 \mathrm{E}-18$ \\
\hline${ }^{241} \mathrm{Am}$ & $2.3 \mathrm{E}-09$ & $7.5 \mathrm{E}-18$ \\
\hline \multicolumn{2}{|l|}{ Total flow (L) } & $3.1 \mathrm{E}+11$ \\
\hline
\end{tabular}

Table 2-5. Radionuclide Air Emissions from 107-N, 100-N Area, in 1993. (Y265)

\begin{tabular}{|c|c|c|}
\hline Radionuclide & 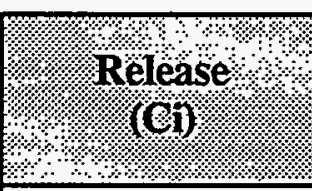 & $\begin{array}{l}\text { Concentration } \\
\text { (feirimi) }\end{array}$ \\
\hline${ }^{60} \mathrm{Co}$ & $3.7 \mathrm{E}-08$ & $3.4 \mathrm{E}-16$ \\
\hline${ }^{90} \mathrm{Sr}$ & $1.0 \mathrm{E}-08$ & $9.3 \mathrm{E}-17$ \\
\hline${ }^{106} \mathrm{Ru}$ & $7.6 \mathrm{E}-07$ & $7.0 \mathrm{E}-15$ \\
\hline${ }^{134} \mathrm{Cs}$ & $6.0 \mathrm{E}-09$ & $5.5 \mathrm{E}-17$ \\
\hline${ }^{137} \mathrm{Cs}$ & $2.4 \mathrm{E}-08$ & $2.2 \mathrm{E}-16$ \\
\hline${ }^{155} \mathrm{Eu}$ & $1.6 \mathrm{E}-08$ & $1.4 \mathrm{E}-16$ \\
\hline${ }^{238} \mathrm{Pu}$ & $6.6 \mathrm{E}-10$ & $6.1 E-18$ \\
\hline${ }^{239,240} \mathrm{Pu}$ & $7.3 \mathrm{E}-10$ & $1.1 \mathrm{E}-17$ \\
\hline \multicolumn{2}{|l|}{ Total flow (L) } & $1.1 \mathrm{E}+11$ \\
\hline
\end{tabular}




\section{WHC-EP-0527-3}

Table 2-6. Radionuclide Air Emissions from 105-KE, 100-KE Area, in 1993. (Y241)

\begin{tabular}{|c|c|c|}
\hline Radionndide & , Release, & $\begin{array}{l}\text { Concentrage } \\
(\mu \mathrm{Gitmb})\end{array}$ \\
\hline${ }^{60} \mathrm{Co}$ & $2.2 \mathrm{E}-06$ & $1.2 \mathrm{E}-14$ \\
\hline${ }^{90} \mathrm{Sr}$ & $5.0 \mathrm{E}-05$ & $2.8 \mathrm{E}-13$ \\
\hline${ }^{106} \mathrm{Ru}$ & $7.6 \mathrm{E}-06$ & $4.3 \mathrm{E}-14$ \\
\hline${ }^{125} \mathrm{Sb}$ & $7.6 \mathrm{E}-07$ & $4.3 \mathrm{E}-15$ \\
\hline${ }^{137} \mathrm{Cs}$ & $1.4 \mathrm{E}-04$ & $7.8 \mathrm{E}-13$ \\
\hline${ }^{154} \mathrm{Eu}$ & $3.9 \mathrm{E}-06$ & $5.3 \mathrm{E}-15$ \\
\hline${ }^{155} \mathrm{Eu}$ & $1.1 \mathrm{E}-06$ & $1.5 \mathrm{E}-15$ \\
\hline${ }^{238} \mathrm{Pu}$ & $9.9 \mathrm{E}-07$ & $5.5 \mathrm{E}-15$ \\
\hline${ }^{239,240} \mathrm{Pu}$ & $7.7 \mathrm{E}-06$ & $4.3 \mathrm{E}-14$ \\
\hline${ }^{241} \mathrm{Am}$ & $5.2 \mathrm{E}-06$ & $2.9 \mathrm{E}-14$ \\
\hline \multicolumn{2}{|l|}{ Total flow (L) } & $7.4 \mathrm{E}+11$ \\
\hline
\end{tabular}

Table 2-7. Radionuclide Air Emissions from 105-KW, 100-KW Area, in 1993. (Y242)

\begin{tabular}{|c|c|c|}
\hline $\begin{array}{c}\text { Radionuclide } \\
{ }^{2}\end{array}$ & $\begin{array}{c}\text { Release } \\
(\mathrm{Ci})\end{array}$ & $\begin{array}{c}\text { Average } \\
\text { Concentration } \\
(\mu \mathrm{Ci} / \mathrm{mL})\end{array}$ \\
\hline${ }^{90} \mathrm{Sr}$ & $1.7 \mathrm{E}-06$ & $2.3 \mathrm{E}-15$ \\
\hline${ }^{106} \mathrm{Ru}$ & $1.6 \mathrm{E}-06$ & $2.2 \mathrm{E}-15$ \\
\hline${ }^{125} \mathrm{Sb}$ & $1.2 \mathrm{E}-06$ & $1.6 \mathrm{E}-15$ \\
\hline${ }^{137} \mathrm{Cs}$ & $2.1 \mathrm{E}-05$ & $2.9 \mathrm{E}-14$ \\
\hline${ }^{154} \mathrm{Eu}$ & $1.9 \mathrm{E}-06$ & $2.6 \mathrm{E}-15$ \\
\hline${ }^{238} \mathrm{Pu}$ & $7.5 \mathrm{E}-09$ & $1.0 \mathrm{E}-17$ \\
\hline${ }^{239,240} \mathrm{Pu}$ & $5.3 \mathrm{E}-08$ & $7.2 \mathrm{E}-17$ \\
\hline${ }^{241} \mathrm{Am}$ & $4.0 \mathrm{E}-08$ & $5.4 \mathrm{E}-17$ \\
\hline \hline Total flow (L) & & $7.4 \mathrm{E}+11$ \\
\hline
\end{tabular}


Table 2-8. Radionuclide Air Emissions from 1706-KE, 100-KE Area, in 1993. (Y243)

\begin{tabular}{|c|c|c|}
\hline Radionvivilide & Release 1 & $\begin{array}{l}\text { A terage } \\
\text { Concentration } \\
(\mu \mathrm{G} / \mathrm{l} \text { III) })\end{array}$ \\
\hline${ }^{90} \mathrm{Sr}$ & $2.4 \mathrm{E}-08$ & $1.3 \mathrm{E}-16$ \\
\hline${ }^{106} \mathrm{Ru}$ & $1.3 E-06$ & $7.3 \mathrm{E}-15$ \\
\hline${ }^{125} \mathrm{Sb}$ & $2.6 \mathrm{E}-07$ & $1.5 \mathrm{E}-15$ \\
\hline${ }^{134} \mathrm{Cs}$ & $7.8 \mathrm{E}-08$ & $4.4 \mathrm{E}-16$ \\
\hline${ }^{155} \mathrm{Eu}$ & $9.1 \mathrm{E}-08$ & $5.1 \mathrm{E}-16$ \\
\hline${ }^{238} \mathrm{Pu}$ & $6.1 \mathrm{E}-10$ & $3.4 \mathrm{E}-18$ \\
\hline${ }^{239,240} \mathrm{Pu}$ & $7.4 \mathrm{E}-09$ & $4.1 \mathrm{E}-17$ \\
\hline \multicolumn{2}{|l|}{ Total flow (L) } & $1.8 \mathrm{E}+11$ \\
\hline
\end{tabular}

Table 2-9. Radionuclide Air Emissions from 1706-KER, 100-KE Area, in 1993. (Y244)

\begin{tabular}{|c|c|c||}
\hline Radionuclide & $\begin{array}{c}\text { Release } \\
(\mathbf{C i})\end{array}$ & $\begin{array}{c}\text { Average } \\
\text { Concentration } \\
(\mu \mathrm{Ci} / \mathrm{mL})\end{array}$ \\
\hline${ }^{90} \mathrm{Sr}$ & $3.3 \mathrm{E}-08$ & $8.9 \mathrm{E}-16$ \\
\hline${ }^{125} \mathrm{Sb}$ & $5.0 \mathrm{E}-08$ & $1.4 \mathrm{E}-15$ \\
\hline${ }^{134} \mathrm{Cs}$ & $4.9 \mathrm{E}-09$ & $1.3 \mathrm{E}-16$ \\
\hline${ }^{137} \mathrm{Cs}$ & $3.4 \mathrm{E}-08$ & $9.3 \mathrm{E}-16$ \\
\hline${ }^{154} \mathrm{Eu}$ & $4.7 \mathrm{E}-08$ & $1.3 \mathrm{E}-15$ \\
\hline${ }^{238} \mathrm{Pu}$ & $3.0 \mathrm{E}-10$ & $8.2 \mathrm{E}-18$ \\
\hline${ }^{239,240} \mathrm{Pu}$ & $1.1 \mathrm{E}-09$ & $3.0 \mathrm{E}-17$ \\
\hline${ }^{241} \mathrm{Am}$ & $6.6 \mathrm{E}-10$ & $1.8 \mathrm{E}-17$ \\
\hline Total Flow (L) & & $3.7 \mathrm{E}+10$ \\
\hline
\end{tabular}


WHC-EP-0527-3

Table 2-10. Radionuclide Air Emissions from 291-A-1, 200 East Area, in 1993. (A552)

\begin{tabular}{|c|c|c|}
\hline Radilonuclidid & 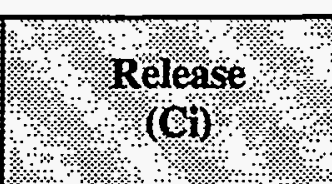 & $\begin{array}{l}\text { A verage } \\
\text { Concentration } \\
\text { (u Girmil) }\end{array}$ \\
\hline${ }^{90} \mathrm{Sr}$ & $4.9 \mathrm{E}-05$ & $3.2 \mathrm{E}-14$ \\
\hline${ }^{106} \mathrm{Ru}$ & $3.6 \mathrm{E}-04$ & $2.4 \mathrm{E}-13$ \\
\hline${ }^{129} \mathrm{~T}$ & $4.8 \mathrm{E}-03$ & $3.2 \mathrm{E}-12$ \\
\hline 137 Cs & $1.4 \mathrm{E}-04$ & $9.2 \mathrm{E}-14$ \\
\hline${ }^{147} \mathrm{Pm}$ & $1.1 \mathrm{E}-04$ & $7.2 \mathrm{E}-14$ \\
\hline${ }^{212} \mathrm{~Pb}$ & $9.7 \mathrm{E}-04$ & $6.4 \mathrm{E}-13$ \\
\hline${ }^{220} \mathrm{Rn}$ & $1.2 \mathrm{E}+01$ & $7.9 \mathrm{E}-09$ \\
\hline${ }^{238} \mathrm{Pu}$ & $3.2 \mathrm{E}-06$ & $2.1 \mathrm{E}-15$ \\
\hline${ }^{239,240} \mathrm{Pu}$ & $4.9 \mathrm{E}-06$ & $3.2 \mathrm{E}-15$ \\
\hline${ }^{241} \mathrm{Pu}$ & $3.3 \mathrm{E}-05$ & $2.2 \mathrm{E}-14$ \\
\hline${ }^{241} \mathrm{Am}$ & $2.7 \mathrm{E}-05$ & $1.7 \mathrm{E}-14$ \\
\hline \multicolumn{2}{|l|}{ Total flc } & $1.5 \mathrm{E}+12$ \\
\hline
\end{tabular}

$$
\mathrm{ND}=\text { none detected }
$$

Table 2-11. Radionuclide Air Emissions from 296-A-1, 200 East Area, in 1993. (A540)

\begin{tabular}{|c|c|c|}
\hline Radionuclide & $\begin{array}{l}3 \text { Release } \\
\text { (Ci) }\end{array}$ & $\begin{array}{c}\text { Ayerage } \\
\text { Concentration } \\
\text { (peirimi) }\end{array}$ \\
\hline${ }^{239,240} \mathrm{Pu}$ & $2.1 \mathrm{E}-06$ & $2.8 \mathrm{E}-14$ \\
\hline${ }^{241} \mathrm{Am}$ & $8.4 \mathrm{E}-07$ & $1.1 \mathrm{E}-14$ \\
\hline \multicolumn{2}{|l|}{ Total flow (L) } & $7.5 \mathrm{E}+10$ \\
\hline
\end{tabular}


Table 2-12. Radionuclide Air Emissions from 296-A-2, . 200 East Area, in 1993. (A542)

\begin{tabular}{|c|c|c|}
\hline Radioniclide & 4.4 Release & $\begin{array}{l}\text { A Yerage } \\
\text { concentration } \\
\text { (Wejming }\end{array}$ \\
\hline $239,240 \mathrm{Pu}$ & ND & $<1.2 \mathrm{E}-15$ \\
\hline${ }^{241} \mathrm{Am}$ & $\mathrm{ND}$ & $<3.8 \mathrm{E}-15$ \\
\hline Total flow $(\mathrm{L})$ & & $7.0 \mathrm{E}+10$ \\
\hline
\end{tabular}

$$
\mathrm{ND}=\text { none detected }
$$

Table 2-13. Radionuclide Air Emissions from 296-A-5A, 200 East Area, in 1993. (A545)

\begin{tabular}{|c|c|c|}
\hline ? Radionuclide & (4) & Concentration \\
\hline $239,240 \mathrm{Pu}$ & $\overline{N D}$ & $<1.8 \mathrm{E}-15$ \\
\hline${ }^{241} \mathrm{Am}$ & $\mathrm{ND}$ & $<6.5 \mathrm{E}-15$ \\
\hline Total flow (L) & & $3.1 \mathrm{E}+11$ \\
\hline
\end{tabular}

$\mathrm{ND}=$ none detected

Table 2-14. Radionuclide Air Emissions from 296-A-5B, 200 East Area, in 1993. (A546)

\begin{tabular}{|c|c|c|}
\hline Radionnclide? & 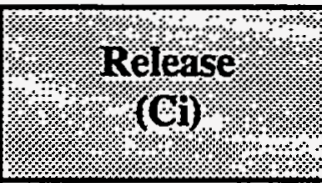 & ( Concentration \\
\hline${ }^{239,240} \mathrm{Pu}$ & ND & $<1.8 \mathrm{E}-15$ \\
\hline${ }^{241} \mathrm{Am}$ & ND & $<6.4 \mathrm{E}-15$ \\
\hline \multicolumn{2}{|l|}{ Total flow (L) } & $3.2 \mathrm{E}+11$ \\
\hline
\end{tabular}

$\mathrm{ND}=$ none detected 
Table 2-15. Radionuclide Air Emissions from 296-A-6, 200 East Area, in 1993. (A547)

\begin{tabular}{|c|c|c|}
\hline Radionuclide: & I Release & 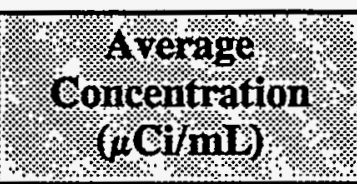 \\
\hline${ }^{239,240} \mathrm{Pu}$ & $\mathrm{ND}$ & $<9.9 \mathrm{E}-16$ \\
\hline${ }^{241} \mathrm{Am}$ & $\mathrm{ND}$ & $<3.5 \mathrm{E}-15$ \\
\hline Total flow (L) & & $1.9 \mathrm{E}+11$ \\
\hline
\end{tabular}

$\mathrm{ND}=$ none detected

Table 2-16. Radionuclide Air Emissions from 296-A-7, 200 East Area, in 1993. (A548)

\begin{tabular}{|c|c|c|}
\hline Radionuclide & Release 4 & $\begin{array}{l}\text { Average } \\
\text { Concentration } \\
\text { (a)ilmil })\end{array}$ \\
\hline${ }^{239,240} \mathrm{Pu}$ & $\mathrm{ND}$ & $<1.0 \mathrm{E}-15$ \\
\hline${ }^{241} \mathrm{Am}$ & $\mathrm{ND}$ & $<3.6 \mathrm{E}-15$ \\
\hline Total flow (L) & & $2.7 \mathrm{E}+11$ \\
\hline
\end{tabular}

$\mathrm{ND}=$ none detected

Table 2-17. Radionuclide Air Emissions from 296-A-8, 200 East Area, in 1993. (A549)

\begin{tabular}{|c|c|c|}
\hline Radionuclide & Release & $\begin{array}{l}\text { Average } \\
\text { Concentration } \\
(\mu \mathrm{Ci} / \mathrm{mL})\end{array}$ \\
\hline${ }^{239,240} \mathrm{Pu}$ & $\mathrm{ND}$ & $<1.0 \mathrm{E}-15$ \\
\hline${ }^{241} \mathrm{Am}$ & ND & $<3.6 \mathrm{E}-15$ \\
\hline Total flow (L) & & $1.3 \mathrm{E}+11$ \\
\hline
\end{tabular}

$\mathrm{ND}=$ none detected 
Table 2-18. Radionuclide Air Emissions from 296-A-17, 200 East Area, in 1993. (E059)

\begin{tabular}{|c|c|c|}
\hline ? Radiominclide & (1) & 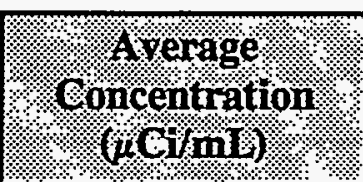 \\
\hline${ }^{90} \mathrm{Sr}$ & $8.2 \mathrm{E}-06$ & $1.2 \mathrm{E}-13$ \\
\hline${ }^{106} \mathrm{Ru}$ & $\overline{\mathrm{ND}}$ & $<3.5 \mathrm{E}-14$ \\
\hline${ }^{129} \mathrm{~T}$ & $4.6 \mathrm{E}-05$ & $6.8 \mathrm{E}-13$ \\
\hline${ }^{137} \mathrm{Cs}$ & $5.3 \mathrm{E}-05$ & $7.9 \mathrm{E}-13$ \\
\hline Total flow $(\mathrm{L})$ & & $6.8 E+10$ \\
\hline
\end{tabular}

$\mathrm{ND}=$ none detected

Table 2-19. Radionuclide Air Emissions from 296-A-21, 200 East Area, in 1993. (E645)

\begin{tabular}{|c|c|c|}
\hline Radionucliate & Release & $\begin{array}{l}\text { A rerage } \\
\text { Concentration } \\
\text { (eO Clmilm) }\end{array}$ \\
\hline $239,240 \mathrm{Pu}$ & ND & $<1.4 \mathrm{E}-15$ \\
\hline${ }^{241} \mathrm{Am}$ & $\mathrm{ND}$ & $<4.8 \mathrm{E}-15$ \\
\hline Total flow (L) & & $2.8 \mathrm{E}+11$ \\
\hline
\end{tabular}

$\mathrm{ND}=$ none detected

Table 2-20. Radionuclide Air Emissions from 296-A-22, 200 East Area, in 1993. (E643)

\begin{tabular}{|c|c|c|}
\hline R. & + Release & $\begin{array}{l}\text { Arerage } \\
\text { Concentration } \\
(\mu \mathrm{eim}, \mathrm{ml})\end{array}$ \\
\hline${ }^{90} \mathrm{Sr}$ & $\overline{\mathrm{ND}}$ & $<1.1 \mathrm{E}-14$ \\
\hline${ }^{137} \mathrm{Cs}$ & ND & $<5.8 \mathrm{E}-14$ \\
\hline $239,240 \mathrm{Pu}$ & ND & $<6.9 \mathrm{E}-15$ \\
\hline${ }^{241} \mathrm{Am}$ & ND & $<1.4 \mathrm{E}-14$ \\
\hline Total flow (L) & & $2.4 E+09$ \\
\hline
\end{tabular}

$\mathrm{ND}=$ none detected 
Table 2-21. Radionuclide Air Emissions from 296-A-27, 200 East Area, in 1993. (E270)

\begin{tabular}{|c|c|c|}
\hline (4) Radionnuclide & : Release & $\begin{array}{l}\text { Concentration } \\
4(\mathrm{H} G \mathrm{mml})\end{array}$ \\
\hline${ }^{90} \mathrm{Sr}$ & $2.7 \mathrm{E}-08$ & $1.7 \mathrm{E}-15$ \\
\hline${ }^{106} \mathrm{Ru}$ & $\overline{\mathrm{ND}}$ & $<4.8 \mathrm{E}-14$ \\
\hline${ }^{129} \mathbf{I}$ & $\mathrm{ND}$ & $<5.7 \mathrm{E}-13$ \\
\hline${ }^{137} \mathrm{Cs}$ & $1.6 \mathrm{E}-07$ & $1.0 \mathrm{E}-14$ \\
\hline \multicolumn{2}{|l|}{ Total flow (L) } & $1.6 \mathrm{E}+10$ \\
\hline
\end{tabular}

$\mathrm{ND}=$ none detected

Table 2-22. Radionuclide Air Emissions from 296-A-29, 200 East Area, in 1993. (E901)

\begin{tabular}{|c|c|c|}
\hline (1) Radionuclide & ?. & $\begin{array}{l}\text { Gonerage } \\
\text { Concentration } \\
(\mu \mathrm{Ci} / \mathrm{mb})\end{array}$ \\
\hline${ }^{90} \mathrm{Sr}$ & $1.2 \mathrm{E}-07$ & $1.1 \mathrm{E}-14$ \\
\hline${ }^{137} \mathrm{Cs}$ & $\mathrm{ND}$ & $<7.3 \mathrm{E}-15$ \\
\hline \multicolumn{2}{|l|}{ Total flow (L) } & $1.1 \mathrm{E}+10$ \\
\hline
\end{tabular}

$\mathrm{ND}=$ none detected

Table 2-23. Radionuclide Air Emissions from 296-A-40, 200 East Area, in 1993. (E013)

\begin{tabular}{|c|c|c|}
\hline Radionuclide & $\begin{array}{l}\text { Release } \\
\text { (Ci) }\end{array}$ & $\begin{array}{c}\text { A verage } \\
\text { Concentration } \\
(\mu \mathrm{Ci} m \mathrm{ml})\end{array}$ \\
\hline${ }^{90} \mathrm{Sr}$ & $6.6 \mathrm{E}-08$ & $5.0 \mathrm{E}-15$ \\
\hline${ }^{106} \mathrm{Ru}$ & $\mathrm{ND}$ & $<6.4 \mathrm{E}-14$ \\
\hline${ }^{129} \mathrm{I}$ & $\mathrm{ND}$ & $<6.2 \mathrm{E}-13$ \\
\hline${ }^{137} \mathrm{Cs}$ & ND & $<7.5 \mathrm{E}-15$ \\
\hline \multicolumn{2}{|l|}{ Total flow (L) } & $1.3 \mathrm{E}+10$ \\
\hline
\end{tabular}

$\mathrm{ND}=$ none detected 
Table 2-24. Radionuclide Air Emissions from 291-B-1, 200 East Area, in 1993. (B691)

\begin{tabular}{|c|c|c|}
\hline Radionuclide. & (2.? Release & 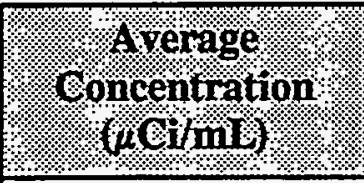 \\
\hline${ }^{90} \mathrm{Sr}$ & $6.5 E-0.5$ & $9.0 \mathrm{E}-14$ \\
\hline${ }^{137} \mathrm{Cs}$ & $1.3 \mathrm{E}-03$ & $1.8 \mathrm{E}-12$ \\
\hline${ }^{239,240} \mathrm{Pu}$ & $4.0 \mathrm{E}-06$ & $5.5 \mathrm{E}-15$ \\
\hline${ }^{241} \mathrm{Am}$ & ND & $<2.7 \mathrm{E}-15$ \\
\hline Total flow (L) & & $7.3 E+11$ \\
\hline
\end{tabular}

$$
\mathrm{ND}=\text { none detected }
$$

Table 2-25. Radionuclide Air Emissions from 296-B-10, 200 East Area, in 1993. (B748)

\begin{tabular}{|c|c|c|}
\hline Radionurelide & $\begin{array}{l}\text { Release } \\
\text { (Ci) }\end{array}$ & 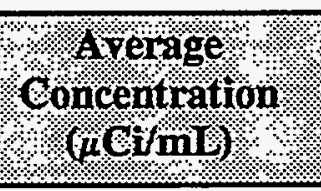 \\
\hline${ }^{90} \mathrm{Sr}$ & $2.6 \mathrm{E}-06$ & $1.1 \mathrm{E}-14$ \\
\hline${ }^{137} \mathrm{Cs}$ & $3.9 \mathrm{E}-06$ & $1.6 \mathrm{E}-14$ \\
\hline $239,240 \mathrm{Pu}$ & $\mathrm{ND}$ & $<1.0 \mathrm{E}-15$ \\
\hline${ }^{241} \mathrm{Am}$ & $\mathrm{ND}$ & $<2.8 \mathrm{E}-15$ \\
\hline Total flow (L) & & $2.4 \mathrm{E}+11$ \\
\hline
\end{tabular}

$\mathrm{ND}=$ none detected

Table 2-26. Radionuclide Air Emissions from 291-S-1, 200 West Area, in 1993. (S006)

\begin{tabular}{|c|c|c|}
\hline Radionuclide & Release & $\begin{array}{l}\text { Ayerage } \\
\text { Concentration } \\
\text { (aciing) }\end{array}$ \\
\hline${ }^{239,240} \mathrm{Pu}$ & $2.8 \mathrm{E}-07$ & $1.0 \mathrm{E}-15$ \\
\hline${ }^{241} \mathrm{Am}$ & ND & $<3.5 \mathrm{E}-15$ \\
\hline Total flow (L) & & $2.8 \mathrm{E}+11$ \\
\hline
\end{tabular}

$\mathrm{ND}=$ none detected 


\section{WHC-EP-0527-3}

Table 2-27. Radionuclide Air Emissions from 296-S-7W; 200 West Area, in 1993. (S016)

\begin{tabular}{|c|c|c|}
\hline Ragionuclide $^{4}$ & 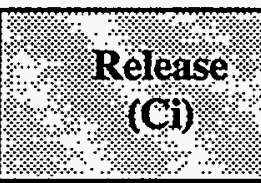 & 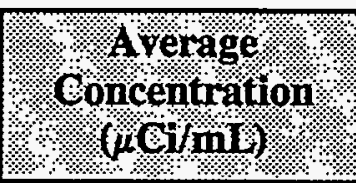 \\
\hline${ }^{239,240} \mathrm{Pu}$ & $7.6 \mathrm{E}-07$ & $5.8 \mathrm{E}-15$ \\
\hline${ }^{241} \mathrm{Am}$ & $7.0 \mathrm{E}-07$ & $5.3 \mathrm{E}-15$ \\
\hline Total flow (L) & & $1.3 \mathrm{E}+11$ \\
\hline
\end{tabular}

Table 2-28. Radionuclide Air Emissions from 296-S-18, 200 West Area, in 1993. (W096)

\begin{tabular}{|c|c|c|}
\hline Radionuclide & $\begin{array}{l}\text { Release } \\
\mathrm{P}^{(\mathrm{Ci})}\end{array}$ & $\begin{array}{l}\text { Concentration } \\
\text { Concion } \\
\text { (ACitml) }\end{array}$ \\
\hline $239,240 \mathrm{Pu}$ & ND & $<1.2 \mathrm{E}-15$ \\
\hline${ }^{241} \mathrm{Am}$ & ND & $<4.0 \mathrm{E}-15$ \\
\hline \multicolumn{2}{|l|}{ Total flow (L) } & $2.5 E+11$ \\
\hline
\end{tabular}

$\mathrm{ND}=$ none detected

Table 2-29. Radionuclide Air Emissions from 296-S-21, 200 West Area, in 1993. (S289)

\begin{tabular}{|c|c|c|}
\hline Radionuclide & $\begin{array}{l}\text { release } \\
\text { Reli) }\end{array}$ & $\begin{array}{l}\text { Average } \\
\text { Concentration } \\
\text { (jeimil })\end{array}$ \\
\hline${ }^{239,240} \mathrm{Pu}$ & ND & $<1.1 \mathrm{E}-15$ \\
\hline${ }^{241} \mathrm{Am}$ & ND & $<3.7 \mathrm{E}-15$ \\
\hline Total flow (L) & & $9.8 \mathrm{E}+11$ \\
\hline
\end{tabular}

$\mathrm{ND}=$ none detected 
Table 2-30. Radionuclide Air Emissions from 296-W-1, 200 West Area, in 1993. (L100)

\begin{tabular}{|c|c|c|}
\hline Radionuclide & (3) & $=4$ A mencentration \\
\hline${ }^{239,240} \mathrm{Pu}$ & $4.4 \mathrm{E}-07$ & $2.1 \mathrm{E}-15$ \\
\hline${ }^{241} \mathrm{Am}$ & $\mathrm{ND}$ & $<4.9 \mathrm{E}-15$ \\
\hline Total flow (L) & & $2.1 \mathrm{E}+11$ \\
\hline
\end{tabular}

$\mathrm{ND}=$ none detected

Table 2-31. Radionuclide Air Emissions from 291-T-1, 200 West Area, in 1993. (T785)

\begin{tabular}{|c|c|c|}
\hline Padionuclide: & Release & $\begin{array}{l}\text { A verage } \\
\text { Concentration } \\
l_{\text {(e) }}\end{array}$ \\
\hline${ }^{90} \mathrm{Sr}$ & $3.4 \mathrm{E}-05$ & $7.7 E-14$ \\
\hline${ }^{137} \mathrm{Cs}$ & $2.5 \mathrm{E}-05$ & $5.6 \mathrm{E}-14$ \\
\hline $239,240 \mathrm{Pu}$ & $2.3 \mathrm{E}-05$ & $5.1 \mathrm{E}-14$ \\
\hline${ }^{241} \mathrm{Am}$ & $4.8 \mathrm{E}-06$ & $1.1 \mathrm{E}-14$ \\
\hline \multicolumn{2}{|l|}{ Total flow (L) } & $4.4 E+11$ \\
\hline
\end{tabular}

Table 2-32. Radionuclide Air Emissions from 296-T-11, 200 West Area, in 1993. (T783)

\begin{tabular}{|c|c|c|}
\hline Radionuclide? & Release & $\begin{array}{l}\text { Average } \\
\text { Concentration } \\
\text { (i) } \mathrm{olmil})\end{array}$ \\
\hline${ }^{239,240} \mathrm{Pu}$ & ND & $<1.0 \mathrm{E}-15$ \\
\hline${ }^{241} \mathrm{Am}$ & ND & $<3.5 \mathrm{E}-15$ \\
\hline Total flow (L) & & $2.4 \mathrm{E}+11$ \\
\hline
\end{tabular}

$\mathrm{ND}=$ none detected 
Table 2-33. Radionuclide Air Emissions from 296-T-12, . 200 West Area, in 1993. (T784)

\begin{tabular}{|c|c|c|}
\hline Radioniclide & Release & $\begin{array}{l}\text { Average } \\
\text { ooncenitration } \\
(1 \mathrm{Cinm})\end{array}$ \\
\hline $239,240 \mathrm{Pu}$ & $\mathrm{ND}$ & $<1.2 \mathrm{E}-14$ \\
\hline $241 \mathrm{Am}$ & $\mathrm{ND}$ & $<2.9 \mathrm{E}-14$ \\
\hline Total flow (L) & & $7.9 E+10$ \\
\hline
\end{tabular}

$$
\mathrm{ND}=\text { none detected }
$$

Table 2-34. Radionuclide Air Emissions from 296-T-13, 200 West Area, in 1993. (T786)

\begin{tabular}{|c|c|c|}
\hline Radionuclífle & 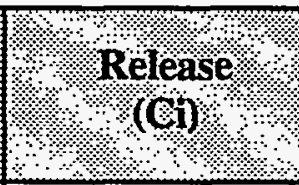 & 3 Concentration \\
\hline${ }^{239,240} \mathrm{Pu}$ & $1.1 \mathrm{E}-05$ & $2.1 \mathrm{E}-13$ \\
\hline${ }^{241} \mathrm{Am}$ & $\mathrm{ND}$ & $<4.5 \mathrm{E}-13$ \\
\hline tal flow & & 5.5 \\
\hline
\end{tabular}

$$
\mathrm{ND}=\text { none detected }
$$

Table 2-35. Radionuclide Air Emissions from 291-U-1, 200 West Area, in 1993. (U771)

\begin{tabular}{|c|c|c|}
\hline $\begin{array}{c}\text { Radionuclide }^{2} \\
{ }^{2}\end{array}$ & $\begin{array}{c}\text { Release } \\
\text { (Ci) }\end{array}$ & $\begin{array}{c}\text { Aterage } \\
\text { Concentration } \\
(\mu \mathrm{Ci} / \mathrm{mI})\end{array}$ \\
\hline${ }^{90} \mathrm{Sr}$ & $2.9 \mathrm{E}-05$ & $8.0 \mathrm{E}-14$ \\
\hline${ }^{137} \mathrm{Cs}$ & $2.0 \mathrm{E}-04$ & $5.5 \mathrm{E}-13$ \\
\hline${ }^{239,240} \mathrm{Pu}$ & $1.9 \mathrm{E}-06$ & $5.3 \mathrm{E}-15$ \\
\hline${ }^{241} \mathrm{Am}$ & $\mathrm{ND}$ & $<4.8 \mathrm{E}-15$ \\
\hline \hline Total flow (L) & $3.6 \mathrm{E}+11$ \\
\hline
\end{tabular}

$\mathrm{ND}=$ none detected 
Table 2-36. Radionuclide Air Emissions from 296-U-2, . 200 West Area, in 1993. (U133)

\begin{tabular}{|c|c|c|}
\hline (4. Radioniclide & $4.4 ?^{(\mathrm{Cl})}$ & (concentration \\
\hline $\mathrm{U}$, total & $3.3 \mathrm{E}-07$ & $5.2 E-14$ \\
\hline \multicolumn{2}{|l|}{ Total flow (L) } & $6.3 \mathrm{E}+09$ \\
\hline
\end{tabular}

Table 2-37. Radionuclide Air Emissions from 296-U-4, 200 West Area, in 1993. (U777)

\begin{tabular}{|c|c|c|}
\hline Radioniclide & (4. & $\begin{array}{l}\text { A Average } \\
\text { Ooncentration } \\
(\mu \mathrm{Ci} \text { mil })\end{array}$ \\
\hline${ }^{90} \mathrm{Sr}$ & $2.6 \mathrm{E}-07$ & $8.0 E-15$ \\
\hline${ }^{067} \mathrm{Ru}$ & $4.0 \mathrm{E}-06$ & $1.2 \mathrm{E}-13$ \\
\hline${ }^{137} \mathrm{Cs}$ & $2.3 \mathrm{E}-07$ & $6.8 \mathrm{E}-15$ \\
\hline $\mathrm{U}$, total & $1.5 \mathrm{E}-0.05$ & $4.5 \mathrm{E}-13$ \\
\hline${ }^{239,240} \mathrm{Pu}$ & $4.3 \mathrm{E}-08$ & $1.3 E-15$ \\
\hline${ }^{241} \mathrm{Am}$ & ND & $<4.8 \mathrm{E}-15$ \\
\hline Total flow (L) & & $3.3 E+10$ \\
\hline
\end{tabular}

$\mathrm{ND}=$ none detected

Table 2-38. Radionuclide Air Emissions from 296-U-13, 200 West Area, in 1993. (U878)

\begin{tabular}{|c|c|c|}
\hline Radionuclide & $\begin{array}{l}1 \\
1\end{array}$ & $\begin{array}{l}\text { average } \\
\text { Concentration } \\
(\mu \text { Cilmi })\end{array}$ \\
\hline $\bar{U}$, total & $1.9 \mathrm{E}-06$ & $3.1 E-13$ \\
\hline Total fl & & +09 \\
\hline
\end{tabular}


WHC-EP-0527-3

Table 2-39. Radionuclide Air Emissions from 291-Z-1, 200 West Area, in 1993. (Z810).

\begin{tabular}{|c|c|c|}
\hline Radionuclide & (: Release & 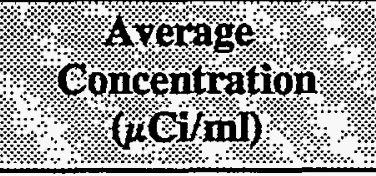 \\
\hline${ }^{238} \mathrm{Pu}$ & ND & $<1.2 \mathrm{E}-14$ \\
\hline $239,240 \mathrm{Pu}$ & $5.1 \mathrm{E}-04$ & $1.4 \mathrm{E}-13$ \\
\hline${ }^{241} \mathrm{Pu}$ & $3.4 \mathrm{E}-03$ & $9.4 \mathrm{E}-13$ \\
\hline${ }^{241} \mathrm{Am}$ & $9.3 \mathrm{E}-05$ & $2.5 \mathrm{E}-14$ \\
\hline \multicolumn{2}{|l|}{ Total flow $(L)$} & $3.7 E+12$ \\
\hline
\end{tabular}

$\mathrm{ND}=$ none detected

Table 2-40. Radionuclide Air Emissions from 296-Z-3, 200 West Area, in 1993. (Z813)

\begin{tabular}{|c|c|c|}
\hline Radionuclide & (1) Release & $\begin{array}{l}\text { Ayerage } \\
\text { Concentration } \\
\text { (jofimi }\end{array}$ \\
\hline${ }^{239,240} \mathrm{Pu}$ & $4.4 \mathrm{E}-07$ & $1.6 \mathrm{E}-14$ \\
\hline${ }^{241} \mathrm{Am}$ & $1.9 \mathrm{E}-07$ & $7.1 \mathrm{E}-15$ \\
\hline Total flow (L) & & $2.7 \mathrm{E}+10$ \\
\hline
\end{tabular}

Table 2-41. Radionuclide Air Emissions from 296-Z-5, 200 West Area, in 1993. (Z913)

\begin{tabular}{|c|c|c|}
\hline Radionuclide & (Ci) & $\begin{array}{c}\text { Aremage } \\
\text { Concentration } \\
(\mu \mathrm{Ci} / \mathrm{ml})\end{array}$ \\
\hline $239,240 \mathrm{Pu}$ & ND & $<9.7 \mathrm{E}-16$ \\
\hline${ }^{241} \mathrm{Am}$ & $7.9 \mathrm{E}-07$ & $5.3 \mathrm{E}-15$ \\
\hline & & 1. \\
\hline
\end{tabular}

$\mathrm{ND}=$ none detected 
Table 2-42. Radionuclide Air Emissions from 308-TRIGA, 300 Area, in 1993.

\begin{tabular}{|c|c|c|}
\hline Radionnclide & : & 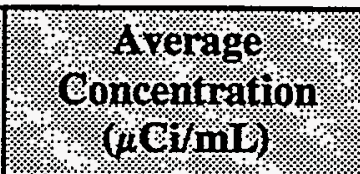 \\
\hline${ }^{131} \mathrm{I}$ & $\mathrm{ND}$ & $<6.2 \mathrm{E}-15$ \\
\hline \multicolumn{2}{|l|}{ Total Flow (L) } & $3.4 \mathrm{E}+10$ \\
\hline
\end{tabular}

$\mathrm{ND}=$ none detected

Table 2-43. Radionuclide Air Emissions from 340-NT-EX, 300 Area, in 1993.

\begin{tabular}{|c|c|c|}
\hline Radionnclide & : & $\begin{array}{l}\text { A Average } \\
\text { Concentration } \\
\text { (H Cimm })\end{array}$ \\
\hline${ }^{131} I$ & $\overline{N D}$ & $<8.7 \mathrm{E}-15$ \\
\hline Total flow (I & & $2.5 \mathrm{E}+10$ \\
\hline
\end{tabular}

$\mathrm{ND}=$ none detected

Table 2-44. Radionuclide Air Emissions from FFTF-CB-EX, 400 Area, in 1993.

\begin{tabular}{|c|c|c|}
\hline Radionuclide & $\begin{array}{l}\text { Release } \\
\text { (Ci) }\end{array}$ & $\begin{array}{l}\text { A A erage } \\
\text { Concentration } \\
\text { (aCilmI) }\end{array}$ \\
\hline${ }^{131} I$ & ND & $<5.8 \mathrm{E}-15$ \\
\hline Total Flow (L) & & $3.6 \mathrm{E}+11$ \\
\hline
\end{tabular}

$\mathrm{ND}=$ none detected

Table 2-45. Radionuclide Air Emissions from FFTF-RE-SB, 400 Area, in 1993.

\begin{tabular}{|c|c|c|}
\hline Radionuclide & Releases? & $\begin{array}{l}\text { Average } \\
\text { Concentration } \\
(\text { e eimi })\end{array}$ \\
\hline${ }^{131} I$ & ND & $<7.4$ E-15 \\
\hline \multicolumn{2}{|l|}{ Total Flow (L) } & $1.9 \mathrm{E}+11$ \\
\hline
\end{tabular}

$\mathrm{ND}=$ none detected 


\section{WHC-EP-0527-3}

Table 2-46. Total Alpha and Total Beta Emissions During 1993 from Stacks Managed by Westinghouse Hanford. (sheet 1 of 4)

\begin{tabular}{|c|c|c|c|c|c|}
\hline \multirow{2}{*}{ 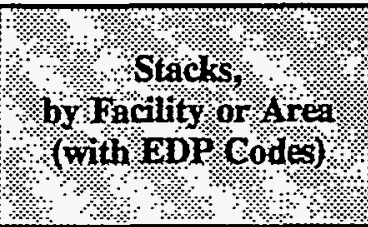 } & \multicolumn{2}{|c|}{$\%$ Kreiease(Ci) } & \multicolumn{2}{|c|}{ Arerage concentrathon } & \multirow{2}{*}{$\begin{array}{l}\text { Yolume } \\
\text { du }\end{array}$} \\
\hline & 铜 & rotal beta & rotal Alpha & Wotal Beta & \\
\hline \multicolumn{6}{|c|}{ 100-N Area } \\
\hline $116-N(Y 211)$ & $4.4 \mathrm{E}-07$ & $2.5 \mathrm{E}-06$ & $4.2 E-16$ & $2.4 \mathrm{E}-15$ & $1.0 \mathrm{E}+12$ \\
\hline 105-N 14' DR (Y259) & $2.7 E-\infty 9$ & $1.2 \mathrm{E}-07$ & $2.8 \mathrm{E}-17$ & $1.3 E-15$ & $9.5 \mathrm{E}+10$ \\
\hline 105-N TA (Y261) & ND & ND & $<-5.4 E-17$ & $-3.0 E-16$ & $3.1 \mathrm{E}+11$ \\
\hline $107-N(Y 265)$ & ND & ND & $<-4.4 E-17$ & $-1.1 E-15$ & $1.1 \mathrm{E}+11$ \\
\hline \multicolumn{6}{|c|}{ 100-K Area } \\
\hline 105-KE (Y241) & $1.5 \mathrm{E}-05$ & $2.9 \mathrm{E}-04$ & $2.0 \mathrm{E}-14$ & $3.9 \mathrm{E}-13$ & $7.5 \mathrm{E}+11$ \\
\hline $105-\mathrm{KW}(\mathrm{Y} 242)$ & $2.7 \mathrm{E}-06$ & $5.3 \mathrm{E}-05$ & $3.6 \mathrm{E}-15$ & $7.1 \mathrm{E}-14$ & $7.5 \mathrm{E}+11$ \\
\hline 1706-KE (Y243) & $1.9 \mathrm{E}-08$ & ND & $1.1 \mathrm{E}-16$ & $<3.4 \mathrm{E}-16$ & $1.8 \mathrm{E}+11$ \\
\hline 1706-KER (Y244) & $1.1 \mathrm{E}-08$ & $1.7 \mathrm{E}-07$ & $3.1 \mathrm{E}-16$ & $4.6 \mathrm{E}-15$ & $3.7 E+10$ \\
\hline \multicolumn{6}{|c|}{ PUREX Plant, 200 East Area } \\
\hline 291-A-1 (A552) & $2.9 \mathrm{E}-05$ & $3.7 \mathrm{E}-04$ & $1.9 \mathrm{E}-14$ & $2.4 E-13$ & $1.5 E+12$ \\
\hline 296-A-1 (A540) & $2.3 E-06$ & $3.4 \mathrm{E}-07$ & $3.1 \mathrm{E}-14$ & $4.5 E-15$ & $7.5 \mathrm{E}+10$ \\
\hline 296-A-2 (A542) & $9.5 E-08$ & ND & $1.4 E-15$ & $-3.3 E-15$ & $7.0 E+10$ \\
\hline 296-A-3 (A543) & $4.4 E-08$ & $4.8 E-07$ & $1.0 \mathrm{E}-15$ & $1.1 \mathrm{E}-14$ & $4.2 E+10$ \\
\hline 296-A-5A (A545) & $2.0 \mathrm{E}-07$ & ND & $6.5 E-16$ & $-7.9 \mathrm{E}-15$ & $3.1 \mathrm{E}+11$ \\
\hline 296-A-5B (A546) & $5.5 \mathrm{E}-08$ & ND & $1.7 E-16$ & $-3.8 E-15$ & $3.2 E+11$ \\
\hline 296-A-6 (A547) & ND & $1.0 \mathrm{E}-07$ & $-1.0 \mathrm{E}-16$ & $5.3 \mathrm{E}-16$ & $1: 9 \mathrm{E}+11$ \\
\hline 296-A-7 (A548) & $1.7 \mathrm{E}-07$ & $2.2 \mathrm{E}-06$ & $6.5 E-16$ & $8.3 \mathrm{E}-15$ & $2.7 E+11$ \\
\hline 296-A-8 (A549) & $1.2 \mathrm{E}-09$ & $2.6 \mathrm{E}-06$ & $9.0 E-18$ & $1.9 \mathrm{E}-15$ & $1.3 E+11$ \\
\hline $296-A-10$ (A550) & ND & ND & $-4.1 E-16$ & $-2.2 E-15$ & $8.2 E+10$ \\
\hline 296-A-14 (A544) & ND & ND & $-4.4 E-16$ & $-2.3 E-15$ & $7.3 E+10$ \\
\hline
\end{tabular}


Table 2-46. Total Alphà and Total Beta Emissions During 1993 from Stacks Managed by Westinghouse Hanford. (sheet 2 of 4)

\begin{tabular}{|c|c|c|c|c|c|}
\hline \multirow{2}{*}{ 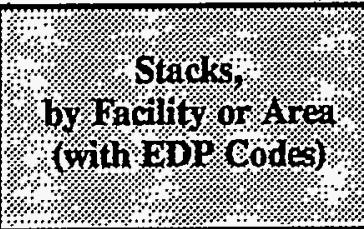 } & \multicolumn{2}{|c|}{ Regesero } & \multicolumn{2}{|c|}{ (1) } & \multirow{2}{*}{ 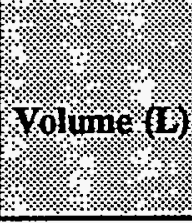 } \\
\hline & yotal & Hotaloxita & rotaly Apla & Mobaik Beta & \\
\hline \multicolumn{6}{|c|}{ B Plant, 200 East Area } \\
\hline 291-B-01 (B691) & $2.4 \mathrm{E}-06$ & $1.6 E-03$ & $3.3 \mathrm{E}-15$ & $2.3 \mathrm{E}-12$ & $7.3 \mathrm{E}+11$ \\
\hline 296-B-05 (B686) & ND & $2.1 E-08$ & $-3.3 \mathrm{E}-16$ & $1.2 \mathrm{E}-15$ & $1.8 \mathrm{E}+10$ \\
\hline 296-B-10 (B748) & ND & $4.4 E-05$ & $-6.4 E-17$ & $1.8 \mathrm{E}-13$ & $2.4 \mathrm{E}+11$ \\
\hline 296-B-13 (B690) & ND & $3.2 \mathrm{E}-08$ & $-1.3 \mathrm{E}-16$ & $3.0 \mathrm{E}-15$ & $1.0 \mathrm{E}+10$ \\
\hline \multicolumn{6}{|c|}{ East Tank Farms, 200 East Area } \\
\hline 296-A-13 (E052) & $2.3 E-08$ & $1.1 \mathrm{E}-07$ & $5.8 \mathrm{E}-16$ & $2.7 \mathrm{E}-15$ & $4.0 E+10$ \\
\hline 296-A-17 (E059) & $5.7 \mathrm{E}-09$ & $5.2 \mathrm{E}-05$ & $8.4 \mathrm{E}-17$ & $7.7 \mathrm{E}-13$ & $6.8 E+10$ \\
\hline 296-A-20 (E197) & ND & $3.0 \mathrm{E}-08$ & $-2.2 \mathrm{E}-16$ & $2.9 \mathrm{E}-15$ & $1.0 \mathrm{E}+10$ \\
\hline 296-A-21 (E645) & ND & ND & $-2.7 E-16$ & $-1.1 E-15$ & $2.8 \mathrm{E}+11$ \\
\hline 296-A-22 (E643) & $1.8 \mathrm{E}-09$ & $3.1 \mathrm{E}-08$ & $7.7 \mathrm{E}-16$ & $1.3 \mathrm{E}-14$ & $2.4 E+09$ \\
\hline 296-A-26 (E297) & ND & ND & $-1.6 E-16$ & $-3.7 E-15$ & $2.5 E+10$ \\
\hline 296-A-27 (E270) & $1.5 E-08$ & $1.2 E-06$ & $9.7 E-16$ & $7.4 \mathrm{E}-14$ & $1.6 \mathrm{E}+10$ \\
\hline 296-A-28 (E272) & $1.1 \mathrm{E}-08$ & $3.6 \mathrm{E}-07$ & $1.9 E-16$ & $6.3 E-15$ & $5.7 E+10$ \\
\hline 296-A-29 (E901) & ND & $1.9 E-06$ & $-3.5 E-16$ & $1.7 \mathrm{E}-13$ & $1.1 E+10$ \\
\hline 296-A-30 (E903) & ND & $4.9 E-09$ & $-1.0 \mathrm{E}-16$ & $1.3 \mathrm{E}-16$ & $3.7 E+10$ \\
\hline 296-A-40 (E013) & ND & ND & $-2.3 E-16$ & $-1.0 E-15$ & $1.3 E+10$ \\
\hline 296-A-41 (E015) & 3.5 E-08 & ND & $2.5 E-16$ & $-2.5 E-15$ & $1.4 \mathrm{E}+11$ \\
\hline 296-B-28 (E886) & $5.2 \mathrm{E}-10$ & $1.3 \mathrm{E}-08$ & $6.3 \mathrm{E}-16$ & $1.5 \mathrm{E}-14$ & $8.2 E+08$ \\
\hline 296-C-05 (E069) & $2.0 \mathrm{E}-08$ & $1.2 \mathrm{E}-06$ & $4.1 \mathrm{E}-16$ & $2.4 \mathrm{E}-14$ & $5.0 E+10$ \\
\hline 296-P-16 (E068) & $1.8 \mathrm{E}-08$ & $1.4 E-05$ & $3.1 \mathrm{E}-16$ & $2.6 \mathrm{E}-13$ & $5.7 \mathrm{E}+10$ \\
\hline 296-P-31 (E209) & $8.2 \mathrm{E}-09$ & ND & $2.8 E-16$ & $-3.3 E-16$ & $3.0 \mathrm{E}+10$ \\
\hline \multicolumn{6}{|c|}{ REDOX, S Plant, and Laundry, 200 West Area } \\
\hline 291-S-01 (S006) & $2.1 \mathrm{E}-07$ & $1.2 E-06$ & $7.6 \mathrm{E}-16$ & 4.3 E-15 & $2.8 \mathrm{E}+11$ \\
\hline $296-\mathrm{S}-02(\mathrm{~S} 032)$ & $6.6 \mathrm{E}-09$ & $2.4 E-08$ & $6.4 E-16$ & $2.3 E-15$ & $1.0 \mathrm{E}+10$ \\
\hline 296-S-04 (S008) & $1.1 \mathrm{E}-07$ & $8.6 \mathrm{E}-07$ & $2.1 \mathrm{E}-15$ & $1.6 \mathrm{E}-14$ & $5.3 \mathrm{E}+10$ \\
\hline $296-S-06$ (S004) & $5.1 \mathrm{E}-09$ & $8.5 \mathrm{E}-08$ & $2.0 \mathrm{E}-15$ & $3.3 \mathrm{E}-14$ & $2.6 \mathrm{E}+09$ \\
\hline
\end{tabular}


Table 2-46. Total Alpha and Total Beta Emissions During 1993 from Stacks Managed by Westinghouse Hanford. (sheet 3 of 4)

\begin{tabular}{|c|c|c|c|c|c|}
\hline \multirow{2}{*}{ 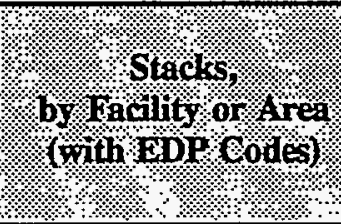 } & \multicolumn{2}{|c|}{ (1) Reiegse $(\mathrm{ci})$ ? } & \multicolumn{2}{|c|}{ Average Concentraition } & \multirow[b]{2}{*}{ Volinine (1) } \\
\hline & \% 1 rotal & Total Befar & Trotal Alphis & Totalo & \\
\hline \multicolumn{6}{|c|}{ REDOX, S Plant, and Laundry (cont.) } \\
\hline 296-S-7W (S016) & $1.1 \mathrm{E}-06$ & ND & $8.2 \mathrm{E}-15$ & $-2.1 \mathrm{E}-15$ & $1.3 \mathrm{E}+11$ \\
\hline $296-S-16$ (S264) & $4.5 \mathrm{E}-09$ & $6.0 \mathrm{E}-08$ & $2.3 \mathrm{E}-15$ & $3.1 \mathrm{E}-14$ & $2.0 \mathrm{E}+09$ \\
\hline 296-S-21 (S289) & ND & ND & $-4.2 \mathrm{E}-16$ & $-7.9 \mathrm{E}-16$ & $9.8 \mathrm{E}+11$ \\
\hline 296-W-01 (L100) & $2.2 \mathrm{E}-07$ & $5.3 \mathrm{E}-06$ & $1.0 \mathrm{E}-15$ & $2.5 \mathrm{E}-14$ & $2.1 \mathrm{E}+11$ \\
\hline \multicolumn{6}{|c|}{ T Plant and TRUSAF, 200 West Area } \\
\hline 291-T-01 (T785) & $2.2 \mathrm{E}-05$ & $9.7 \mathrm{E}-05$ & $5.0 \mathrm{E}-14$ & $2.2 \mathrm{E}-13$ & $4.4 \mathrm{E}+11$ \\
\hline 296-T-11 (T783) & $3.3 \mathrm{E}-08$ & $1.0 \mathrm{E}-06$ & $1.4 \mathrm{E}-16$ & $4.3 \mathrm{E}-15$ & $2.4 \mathrm{E}+11$ \\
\hline $296-\mathrm{T}-12$ (T784) & ND & ND & $-1.4 \mathrm{E}-15$ & $-2.1 \mathrm{E}-14$ & $7.9 \mathrm{E}+10$ \\
\hline $296-\mathrm{T}-13$ (T786) & $1.8 \mathrm{E}-06$ & $5.8 \mathrm{E}-06$ & $3.2 \mathrm{E}-14$ & $1.1 \mathrm{E}-13$ & $5.5 \mathrm{E}+10$ \\
\hline \multicolumn{6}{|c|}{ U Plant and $\mathrm{UO}_{3}, 200$ West Area } \\
\hline 291-U-01 (U771) & $7.3 \mathrm{E}-07$ & $3.1 \mathrm{E}-04$ & $2.0 \mathrm{E}-15$ & $8.5 E-13$ & $3.6 \mathrm{E}+11$ \\
\hline 296-U-02 (U133) & $2.6 \mathrm{E}-07$ & $4.1 \mathrm{E}-07$ & $4.1 \mathrm{E}-14$ & $6.4 \mathrm{E}-14$ & $6.3 \mathrm{E}+09$ \\
\hline 296-U-04 (U777) & $5.0 \mathrm{E}-06$ & $2.7 \mathrm{E}-05$ & $1.5 \mathrm{E}-13$ & $8.1 E-13$ & $3.3 \mathrm{E}+10$ \\
\hline 296-U-13 (U878) & $3.1 \mathrm{E}-08$ & ND & $4.9 \mathrm{E}-15$ & $-4.6 \mathrm{E}-15$ & $6.3 \mathrm{E}+09$ \\
\hline \multicolumn{6}{|c|}{ West Tank Farms, 200 West Area } \\
\hline 296-P-22 (W191) & ND & ND & $-1.2 \mathrm{E}-16$ & $-2.7 E-15$ & $9.3 \mathrm{E}+09$ \\
\hline 296-P-23 (W190) & $8.1 \mathrm{E}-09$ & $7.0 \mathrm{E}-07$ & $5.3 \mathrm{E}-16$ & $4.6 \mathrm{E}-14$ & $1.5 \mathrm{E}+10$ \\
\hline 296-S-15 (W111) & ND & $9.1 \mathrm{E}-08$ & $-4.4 \mathrm{E}-17$ & $1.9 \mathrm{E}-15$ & $4.8 \mathrm{E}+10$ \\
\hline 296-S-18 (W096) & $2.4 \mathrm{E}-08$ & $2.7 \mathrm{E}-07$ & $9.7 \mathrm{E}-17$ & $1.1 \mathrm{E}-15$ & $2.5 \mathrm{E}+11$ \\
\hline $296-\mathrm{T}-17$ (W117) & $1.3 \mathrm{E}-09$ & ND & $8.6 \mathrm{E}-17$ & $-2.0 \mathrm{E}-15$ & $1.5 \mathrm{E}+10$ \\
\hline 296-T-18 (W882) & $1.8 \mathrm{E}-09$ & $5.4 \mathrm{E}-08$ & $1.1 \mathrm{E}-15$ & $3.5 \mathrm{E}-14$ & $1.6 \mathrm{E}+09$ \\
\hline 296-W-03 (W003) & ND & $1.1 \mathrm{E}-08$ & $-4.1 \mathrm{E}-16$ & $-3.3 \mathrm{E}-16$ & $3.4 \mathrm{E}+10$ \\
\hline \multicolumn{6}{|c|}{ Z Plant, 200 West Area } \\
\hline 291-Z-01 (Z810) & $6.4 \mathrm{E}-0.4$ & $4.4 E-05$ & $1.7 \mathrm{E}-13$ & $1.2 \mathrm{E}-14$ & $3.7 \mathrm{E}+12$ \\
\hline 296-Z-03 (Z813) & $1.0 \mathrm{E}-06$ & $4.5 \mathrm{E}-08$ & $3.7 \mathrm{E}-14$ & $1.6 \mathrm{E}-15$ & $2.7 \mathrm{E}+10$ \\
\hline $296-Z-05(Z 913)$ & $\mathrm{ND}$ & ND & $-2.4 E-17$ & $-3.1 E-15$ & $1.5 \mathrm{E}+11$ \\
\hline
\end{tabular}




\section{WHC-EP-0527-3}

Table 2-46. Total Alpha and Total Beta Emissions During 1993 from Stacks Managed by Westinghouse Hanford. (sheet 4 of 4)

\begin{tabular}{|c|c|c|c|c|c|}
\hline \multirow{2}{*}{ \% } & \multicolumn{2}{|c|}{ Reserse $(\mathrm{si)})$} & \multicolumn{2}{|c|}{ Ayerage Concentrifion } & \multirow{2}{*}{ Yolimento } \\
\hline & Alotal & Total Beta & Total apha & Iotal Beta & \\
\hline \multicolumn{6}{|c|}{ Z Plant (cont.) } \\
\hline $296-Z-06$ (Z802) & $3.3 \mathrm{E}-08$ & ND & $2.5 \mathrm{E}-16$ & $-2.0 \mathrm{E}-15$ & $1.4 \mathrm{E}+11$ \\
\hline 296-Z-14 (Z814) & $3.7 E-09$ & ND & $1.5 \mathrm{E}-16$ & $-4.2 \mathrm{E}-16$ & $2.5 \mathrm{E}+10$ \\
\hline \multicolumn{6}{|c|}{300 Area } \\
\hline 340-NT-EX & $1.3 \mathrm{E}-08$ & $5.5 \mathrm{E}-08$ & $5.1 \mathrm{E}-16$ & $2.2 \mathrm{E}-15$ & $2.5 \mathrm{E}+10$ \\
\hline 306E-ULAB & $3.6 \mathrm{E}-08$ & $1.1 \mathrm{E}-07$ & $9.0 E-16$ & $2.9 \mathrm{E}-15$ & $3.9 \mathrm{E}+10$ \\
\hline 308-GL-EX & $2.7 \mathrm{E}-08$ & $1.8 \mathrm{E}-07$ & $5.5 \mathrm{E}-16$ & $3.5 \mathrm{E}-15$ & $5.0 \mathrm{E}+10$ \\
\hline 308-ET-EX & $4.4 \mathrm{E}-08$ & $1.5 \mathrm{E}-07$ & $8.4 E-16$ & $2.9 \mathrm{E}-15$ & $5.2 \mathrm{E}+10$ \\
\hline 308-TRIGA & $4.0 \mathrm{E}-08$ & $1.1 \mathrm{E}-07$ & $1.2 \mathrm{E}-15$ & $3.2 \mathrm{E}-15$ & $3.4 E+10$ \\
\hline 377-SGEF & $7.7 \mathrm{E}-08$ & $3.3 \mathrm{E}-07$ & $1.7 \mathrm{E}-15$ & $7.3 \mathrm{E}-15$ & $4.5 \mathrm{E}+10$ \\
\hline \multicolumn{6}{|c|}{400 Area } \\
\hline FFTF-CB-EX & $5.9 \mathrm{E}-07$ & $2.3 \mathrm{E}-06^{*}$ & $1.6 \mathrm{E}-15$ & $6.5 \mathrm{E}-15$ & $3.6 \mathrm{E}+11$ \\
\hline FFTF-RE-SB & $1.4 \mathrm{E}-06$ & $4.5 \mathrm{E}-06^{*}$ & $7.3 \mathrm{E}-15$ & $2.4 \mathrm{E}-14$ & $1.9 \mathrm{E}+11$ \\
\hline FFTF-HT-TR & $2.3 E-07$ & $8.2 E-07^{*}$ & $3.7 \mathrm{E}-15$ & $1.3 \mathrm{E}-14$ & $6.2 \mathrm{E}+10$ \\
\hline 437-MN\&ST & $1.6 E-07$ & $6.0 \mathrm{E}-07^{*}$ & $6.9 \mathrm{E}-16$ & $2.5 \mathrm{E}-15$ & $2.4 \mathrm{E}+11$ \\
\hline
\end{tabular}

Total beta assumed to be ${ }^{137} \mathrm{Cs}$ for dose calculations.

EDP = electronic data processing

ND $=$ none detected

$\mathrm{TA}=$ transfer area

$\mathrm{DR}=$ decontamination room 


\section{WHC-EP-0527-3}

Table 2-47. Nonradioactive Constituents in Air Emissions During 1992 from Facilities Managed by Westinghouse Hanford.

\begin{tabular}{|c|c|c|c|c|c|c|c|}
\hline \multirow{2}{*}{ H. } & \multirow{2}{*}{ Consungution } & (2) & ?. & \multicolumn{2}{|c|}{ Gonshinent $($ hg) } & \multicolumn{2}{|c|}{ 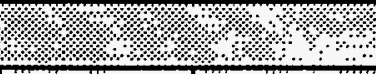 } \\
\hline & & rintculates & $\mathrm{SO}_{3}$ & $\mathrm{No} * \%$ & $\mathrm{co}$ & $4.0 \%$ & thed \\
\hline $\begin{array}{l}200 \text { East Area } \\
\text { Powerhouse }\end{array}$ & $2.3 \mathrm{E}+07 \mathrm{~kg}$ & $7.5 \mathrm{E}+03$ & $2.1 \mathrm{E}+05$ & $1.6 \mathrm{E}+05$ & $5.8 \mathrm{E}+04$ & $5.5 \mathrm{E}+02$ & $1.4 \mathrm{E}+02$ \\
\hline $\begin{array}{l}200 \text { West Area } \\
\text { Powerhouse }\end{array}$ & $2.1 \mathrm{E}+07 \mathrm{~kg}$ & $1.4 \mathrm{E}+03$ & $1.9 \mathrm{E}+05$ & $1.5 \mathrm{E}+05$ & $5.3 \mathrm{E}+04$ & $5.5 \mathrm{E}+02$ & $1.4 \mathrm{E}+02$ \\
\hline $\begin{array}{l}300 \text { Area } \\
\text { Powerhouses }\end{array}$ & $\begin{array}{c}9.4 \mathrm{E}+06 \mathrm{~L} \\
(2.4 \mathrm{E}+06 \mathrm{gal})\end{array}$ & $2.1 \mathrm{E}+0.4$ & $3.0 \mathrm{E}+05$ & $6.0 \mathrm{E}+04$ & $5.4 E+03$ & $2.0 \mathrm{E}+02$ & $1.8 \mathrm{E}+01$ \\
\hline $\begin{array}{l}1100 \text { Area } \\
\text { Heating Plant }\end{array}$ & & & & operate) & & & \\
\hline
\end{tabular}

$\mathrm{NO}_{\mathrm{x}}=$ nitrogen oxides

$\mathrm{SO}_{\mathrm{x}}=$ sulfur oxides

$\mathrm{CO}=$ carbon monoxide

VOC $=$ volatile organic compounds 


\subsection{LQUID EFFLUENTS}

The majority of liquid effluents discharged to the environment from facilites managed by Westinghouse Hanford enter the soil at the Hanford Site, with the balance discharging to the Columbia River. Data on these effluents, categorized as either radioactive or nonradioactive, are presented in this section.

\subsection{RADIOACTIVE LIQUID EFFLUENTS}

Westinghouse Hanford-managed facilities discharge liquid effluents potentially or normally contaminated with radionuclides to the soil or the Columbia River. The general categories of these liquid effluents are: (1) cooling water, (2) steam condensates, (3) process condensates, (4) laboratory and chemical sewer waste water, (5) laundry waste water, (6) filter backwash, and (7) groundwater seepage resulting from discharges to the soil.

The measured quantities of radionuclides discharged in liquid effluents from facilities, by area, managed by Westinghouse Hanford are summarized in Table 3-1. Releases of specific radionuclides and total activity discharged by individual liquid effluent streams are presented in Tables 3-2 through 3-17. Included in these tables are releases to the Columbia River adjacent to 100-N Area via groundwater seepage along the stretch of riverbank known as the N Springs (see Figure 3-1).

Figure 3-1. Liquid Effluent Releases of ${ }^{90} \mathrm{Sr}$ to the Columbia River from 1987 - 1993.

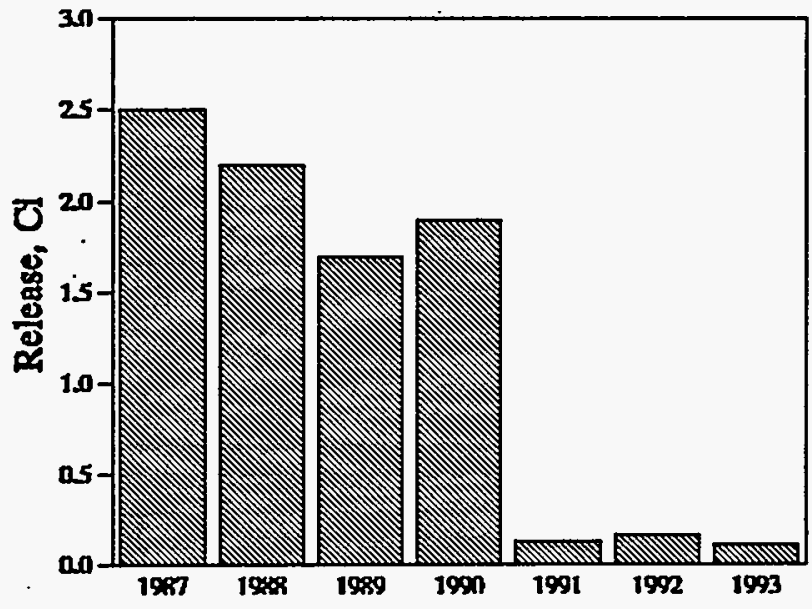




\subsubsection{Radioactive Constituents in Liquid Effluents}

In the 100-N Area, total radionuclide releases continued to be far below past releases during $\mathrm{N}$ Reactor operation.

In the 200 Areas, the PUREX Plant, which has not operated since March 1990, did not discharge radionuclides in quantities significantly different than were discharged in 1992. The PUREX Plant chemical sewer waste water (CSL), can be diverted either automatically or manually to the 216-A-42 Retention Basin, which prevents the effluents from discharging to the soil. Diversion normally would occur when online radiation or $\mathrm{pH}$ monitors upstream of the diversion valve register potential or real upset conditions in the effluent streams. The 216-A-42 Retention Basin allows sampling and analyzing of diverted suspect effluent while it is temporarily retained there. If analysis demonstrates compliance with release limits, the effluent is discharged to the 216-B-3 Pond. If the effluent is not within applicable release limits, it can be pumped to the PUREX Plant for reprocessing. The CWL and SCD release points did not discharge liquid effluents in 1993. The calendar year 1992 CWL flow was incorrectly reported in the calendar year 1992 release report, WHC-EP-0527-2 (Diediker 1993). Table 3-16 on page 3-12 reported the total CWL flow as $1.6 \mathrm{E}+09 \mathrm{~L}$ (4.1 E+08 gal.). The calendar year 1992 total flow from CWL should be $6.6 \mathrm{E}+07 \mathrm{~L}$. (1.7 $\mathrm{E}+07 \mathrm{gal})$.

Although the 242-A Evaporator-Crystallizer did not operate in 1993 to process liquid radioactive material, some effluents were discharged to the 216-B-3 Pond as a result of testing required before scheduled operations resume in 1994.

B Plant operations and the 241-AY and -AZ Tank Farms did not discharge any process condensate or steam-coil condensate, respectively. On February 27, 1992, the B Plant chemical sewer discharge (BCE) to the 216-B-63 Trench was combined with the B Plant cooling water discharge (CBC), which discharges to the 216-B-3 Pond, therefore no data for the $\mathrm{CBC}$ discharge point is presented in this report.

Discharges to French drains and from the two sources at $T$ Plant are not sampled or recorded for flow. This is because the effluents are judged not to have a potential of exceeding $4 \%$ of the DCG values on a yearly average. Four percent of a DCG is equivalent to $4 \mathrm{mrem}$, which is the drinking water standard (Radiological Drinking Water Standards: U.S. Environmental Protection Agency, National Primary Drinking Water Regulations [40 CFR 141]; and State of Washington, Rules and Regulations of the State Board of Health Regarding Public Water Systems [WAC 248-54]). In 1993, the 200 Area facilities continued to generate the largest number of effluent streams, with related volumes and radioactivity, that discharge to the soil.

In the 300 Area, radionuclide releases did not change significantly from releases in earlier years. 


\subsubsection{Nonradioactive Constituents in Radioactive Liquid Effluents}

A new liquid effluent report is being published for the first time in 1993, covering CY 1993 nonradioactive liquid effluents. This document, Liquid Effluent Annual Report, (WHC 1994) provides descriptions of 22 liquid effluent waste streams. The report provides details on the processes contributing to the waste stream, information on the volume, summaries of the sampling requirements, and the analytical data.

\subsection{NONRADIOACTIVE LIQUID EFFLUENTS}

\subsubsection{National Pollutant Discharge Elimination System Discharges From the 100 Areas}

Liquid effluents discharged to the Columbia River from the 100-N and 100-K Areas are regulated by the parameters in the NPDES permit for the Hanford Site. A list of the discharge points is provided in Table 3-18 a summary of analyses performed to ensure compliance with the NPDES permit is given Table 3-19.

\subsubsection{Sanitary Sewage Discharges to the Soil}

Various facilities managed by Westinghouse Hanford discharged sanitary sewage. In the 100-N Area, sanitary waste water was discharged to the 100-N Sewage Lagoon and five septic tanks. In the 100-B, 100-D, 100-H, and 100-K Areas, sanitary sewage was discharged to septic tanks and drainfields. In the 200 Areas, sanitary waste water was discharged to a system of septic tanks and drainfields. Sludge was pumped from septic tanks in the 200 Areas and taken to the 100-N Sewage Lagoon for disposal. In the 300 Area, sanitary sewage was discharged to the 300 Area septic tank and trench system. In the 400 Area, sanitary sewage was discharged to a septic system. The volume of sewage discharged by area during 1993 is shown in Table 3-20.

\subsubsection{Process Water Discharges}

Westinghouse Hanford-managed facilities discharged nonhazardous waste water to the ground at the Hanford Site. The 200 West Area Water Treatment Facility discharged waste water totalling $2.5 \mathrm{E}+07 \mathrm{~L}(6.5 \mathrm{E}+06 \mathrm{gal})$ to percolation ponds.

Approximately $4.2 \mathrm{E}+08 \mathrm{~L}$ (1.1 E+08 gal) of nonhazardous nonradioactive liquid wastes were discharged to the 300 Area process trenches from the 300 Area Process Sewer System.

In the 400 Area, approximately $3.5 \mathrm{E}+07 \mathrm{~L}(9.2 \mathrm{E}+06 \mathrm{gal})$ of process nonhazardous nonradioactive waste water from the 400 Area Process Sewer drained to a process pond located north of the 400 Area. Approximately $8.3 \mathrm{E}+04 \mathrm{~L}$ (2.2 E+04 gal) of liquid wastes (principally condensate and water softener waste) drained to 24 drywells. 
Facilities managed by Westinghouse Hanford did not discharge to the City of Richland publicly owned treatment works (POTW) any waste water that could be classified as a significant industrial discharge. Significant industrial discharges are defined as those meeting any of the following criteria:

- Being subject to national pretreatment standards promulgated under Section 307 (b) or (c) of the Clean Water Act of 1977

-Having any of the priority toxic pollutants listed in 40 CFR 403 (EPA 1992)

-Having toxic pollutants as defined in the Clean Water Act of 1977, Section 307

-Having a discharge flow of $1.5 \mathrm{E}+05 \mathrm{~L}$ (4.0 E+04 gal) or more per average workday

-Having a flow greater than $5 \%$ of the flow of the POTW.

Such discharges are prohibited unless a permit is issued by the City of Richland. 
Table 3-1. Radionuclides in Liquid Effluents Discharged to Soil at the Hanford Site in 1993 from Facilities, by Area, Managed by Westinghouse Hanford.

\begin{tabular}{|c|c|c|c|c|}
\hline \multirow{2}{*}{ Rerdinonichide } & \multicolumn{4}{|c|}{ 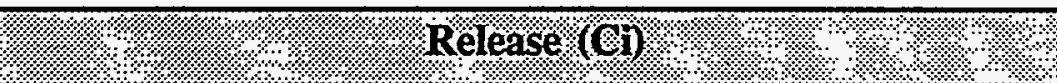 } \\
\hline & 200\%4reas. & 300 Anrea & 400 Arrea & Total \\
\hline${ }^{3} \mathrm{H}$ & $1.5 \mathrm{E}+01$ & & & $1.5 \mathrm{E}+01$ \\
\hline${ }^{90} \mathrm{Sr}$ & $7.1 \mathrm{E}-02$ & $2.2 \mathrm{E}-02^{\mathrm{a}}$ & & $9.3 \mathrm{E}-02$ \\
\hline${ }^{99} \mathrm{Tc}$ & $8.5 \mathrm{E}-04$ & & & $8.5 \mathrm{E}-04$ \\
\hline${ }^{137} \mathrm{Cs}$ & $1.7 \mathrm{E}-02$ & & $1.5 \mathrm{E}-02^{2}$ & $1.9 \mathrm{E}-02$ \\
\hline $\mathrm{U}$, total & $1.7 \mathrm{E}-03$ & $5.2 \mathrm{E}-03^{b}$ & & $6.9 \mathrm{E}-03$ \\
\hline${ }^{238} \mathrm{Pu}$ & $1.4 \mathrm{E}-03$ & & & $1.4 \mathrm{E}-03$ \\
\hline $239,240 \mathrm{Pu}$ & $1.8 \mathrm{E}-02$ & & & $1.8 \mathrm{E}-02$ \\
\hline${ }^{241} \mathrm{Pu}$ & $2.4 \mathrm{E}-02$ & & & $2.4 \mathrm{E}-02$ \\
\hline$\overline{{ }^{241} \mathrm{Am}}$ & $2.8 \mathrm{E}-03$ & & & $2.8 \mathrm{E}-03$ \\
\hline
\end{tabular}

'This value represents total beta activity.

'This value represents total alpha activity. 
Table 3-2. Radionuclide Discharges via N Springs, 100-N Area, to Columbia River in 1993. (Y101)

\begin{tabular}{|c|c|c|}
\hline Radionurlide? & $\begin{array}{l}\text { Release } \\
4:(\mathrm{Ci})\end{array}$ & $\begin{array}{l}\text { Ayerage } \\
\text { Concentration } \\
(\text { Ueirml })\end{array}$ \\
\hline${ }^{3} \mathrm{H}$ & $3.8 \mathrm{E}-01$ & $1.9 \mathrm{E}-05$ \\
\hline${ }^{60} \mathrm{Co}$ & $8.8 \mathrm{E}-05$ & $4.4 \mathrm{E}-09$ \\
\hline${ }^{90} \mathrm{Sr}$ & $1.1 \mathrm{E}-01$ & $5.5 \mathrm{E}-06$ \\
\hline${ }^{106 \mathrm{Ru}}$ & $8.7 \mathrm{E}-05$ & $4.3 \mathrm{E}-09$ \\
\hline${ }^{125} \mathrm{Sb}$ & $7.7 \mathrm{E}-05$ & $3.8 \mathrm{E}-09$ \\
\hline$\overline{137} \mathrm{Cs}$ & $4.0 \mathrm{E}-05$ & $2.0 \mathrm{E}-09$ \\
\hline${ }^{239,240} \mathrm{Pu}$ & $1.4 \mathrm{E}-07$ & $7.0 \mathrm{E}-12$ \\
\hline Flow & \multicolumn{2}{|c|}{$2.0 \mathrm{E}+07 \mathrm{~L}(5.3 \mathrm{E}+06 \mathrm{gal})$} \\
\hline
\end{tabular}

Table 3-3 Radionuclide Discharges via National Pollutant Discharge Elimination System Outfall 009 (102-in. Outfall), 100-N Area, to Columbia River in 1993. (Y120)

\begin{tabular}{|c|c|c|}
\hline Rationuclide & $\begin{array}{l}\text { Release } \\
\text { P }\end{array}$ & $\begin{array}{l}\text { A A rerage } \\
\text { Concentration } \\
\text { (a ci/min) }\end{array}$ \\
\hline${ }^{125} \mathrm{Sb}$ & $5.7 \mathrm{E}-05$ & $4.3 \mathrm{E}-09$ \\
\hline Flow & \multicolumn{2}{|c|}{$1.3 \mathrm{E}+07 \mathrm{~L}(3.5 \mathrm{E}+06 \mathrm{gal})$} \\
\hline
\end{tabular}


Table 3-4. Radionuclide Discharges via National Pollutant Discharge Elimination System Outfall 004 (1908-K Outfall), 100-K Area, to Columbia River in 1993. (Y130)

\begin{tabular}{|c|c|c|}
\hline I: & ( $=$ & $\begin{array}{l}\text { Average } \\
\text { Concentration } \\
(y-1 m i n t)\end{array}$ \\
\hline${ }^{60} \mathrm{Co}$ & $2.7 \mathrm{E}-04$ & $3.0 \mathrm{E}-10$ \\
\hline${ }^{106} \mathrm{Ru}$ & $1.5 \mathrm{E}-03$ & $1.7 \mathrm{E}-09$ \\
\hline${ }^{134} \mathrm{Cs}$ & $4.7 E-05$ & $5.2 \mathrm{E}-11$ \\
\hline${ }^{137} \mathrm{Cs}$ & $4.0 \mathrm{E}-04$ & $4.4 \mathrm{E}-10$ \\
\hline Flow & \multicolumn{2}{|c|}{$9.1 \mathrm{E}+08 \mathrm{~L}(2.4 \mathrm{E}+08 \mathrm{gal})$} \\
\hline
\end{tabular}

Table 3-5. Radionuclides in 222-S Laboratory Chemical Sewer Discharges (207-SL) to 216-S-26 Crib, 200 West Area, in 1993. (H101)

\begin{tabular}{|c|c|c|}
\hline 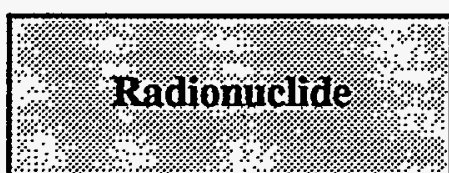 & Release & 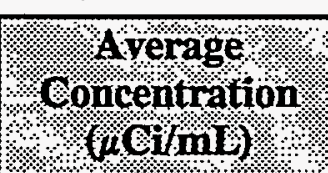 \\
\hline${ }^{90} \mathrm{Sr}$ & $3.6 \mathrm{E}-05$ & $5.6 \mathrm{E}-09$ \\
\hline${ }^{137} \mathrm{Cs}$ & $\overline{\mathrm{ND}}$ & $<3.5 \mathrm{E}-08$ \\
\hline${ }^{239,240} \mathrm{Pu}$ & $\mathrm{ND}$ & $<5.2 \mathrm{E}-09$ \\
\hline$\overline{241} \mathrm{Am}$ & ND & $<1.0 \mathrm{E}-08$ \\
\hline Total $\alpha$ & $6.2 \mathrm{E}-06$ & $9.7 \mathrm{E}-10$ \\
\hline Total B & $1.7 \mathrm{E}-04$ & $2.7 \mathrm{E}-08$ \\
\hline Flow & \multicolumn{2}{|c|}{$6.5 \mathrm{E}+06 \mathrm{~L}(1.7 \mathrm{E}+06 \mathrm{gal})$} \\
\hline
\end{tabular}

$\mathrm{ND}=$ none detected 
Table 3-6. Radionuclides in $\mathrm{UO}_{3}$ Plant Discharges (207-U) to 216-U-14 Ditch, 200 West Area, in 1993.

(H102)

\begin{tabular}{|c|c|c|}
\hline Radionuclide & $\begin{array}{l}\text { Release } \\
\text { (Gi) }\end{array}$ & $\begin{array}{l}\text { Ayerage } \\
\text { Concentration } \\
(\mu \mathrm{cimi})\end{array}$ \\
\hline${ }^{3} \mathrm{H}$ & $1.2 \mathrm{E}-01$ & $4.3 \mathrm{E}-07$ \\
\hline${ }^{90} \mathrm{Sr}$ & $4.4 \mathrm{E}-03$ & $1.6 \mathrm{E}-08$ \\
\hline${ }^{99} \mathrm{Tc}$ & ND & $<1.7 \mathrm{E}-07$ \\
\hline${ }^{137} \mathrm{Cs}$ & $1.6 \mathrm{E}-02$ & $5.8 \mathrm{E}-08$ \\
\hline $\mathrm{U}$, total & $9.7 \mathrm{E}-04$ & $3.6 \mathrm{E}-09$ \\
\hline $239,240 \mathrm{Pu}$ & $\overline{\mathrm{ND}}$ & $<1.4 \mathrm{E}-08$ \\
\hline$\overline{241} \mathrm{Am}$ & $\overline{\mathrm{ND}}$ & $<2.7 \mathrm{E}-08$ \\
\hline Total $\alpha$ & $2.3 \mathrm{E}-03$ & $8.5 \mathrm{E}-09$ \\
\hline Total B & $9.4 \mathrm{E}-03$ & $3.5 \mathrm{E}-08$ \\
\hline Flow & \multicolumn{2}{|c|}{$2.7 \mathrm{E}+08 \mathrm{~L}(7.1 \mathrm{E}+07 \mathrm{gal})$} \\
\hline
\end{tabular}

$\mathrm{ND}=$ none detected

Table 3-7. Radionuclides in Plutonium Finishing Plant Discharges (2904-ZA) to 216-Z-20 Crib, 200 West Area, in 1993. (H103)

\begin{tabular}{|c|c|c|}
\hline \multirow{2}{*}{$\begin{array}{c}\text { Radionuclide- } \\
\text { Release } \\
(\mathrm{Ci})\end{array}$} & $\begin{array}{c}\text { A Aerage } \\
\text { Concentration } \\
(j \mathrm{Ci} / \mathrm{mL})\end{array}$ \\
\hline${ }^{90} \mathrm{Sr}$ & $1.1 \mathrm{E}-03$ & $1.1 \mathrm{E}-08$ \\
\hline${ }^{137} \mathrm{Cs}$ & $\mathrm{ND}$ & $<4.2 \mathrm{E}-08$ \\
\hline${ }^{238} \mathrm{Pu}$ & $1.4 \mathrm{E}-03$ & $1.4 \mathrm{E}-08$ \\
\hline${ }^{239,240} \mathrm{Pu}$ & $3.6 \mathrm{E}-03$ & $3.5 \mathrm{E}-08$ \\
\hline${ }^{241} \mathrm{Pu}$ & $2.4 \mathrm{E}-02$ & $2.4 \mathrm{E}-07$ \\
\hline${ }^{241} \mathrm{Am}$ & $2.8 \mathrm{E}-03$ & $2.8 \mathrm{E}-08$ \\
\hline Total $\alpha$ & $2.4 \mathrm{E}-03$ & $2.4 \mathrm{E}-08$ \\
\hline Total $B$ & $2.5 \mathrm{E}-03$ & $2.5 \mathrm{E}-08$ \\
\hline Flow & $1.0 \mathrm{E}+08 \mathrm{~L}(2.7 \mathrm{E}+07 \mathrm{gal})$ \\
\hline
\end{tabular}

$\mathrm{ND}=$ none detected 
Table 3-8. Radionuclides in Discharges from PlutoniumUranium Extraction Plant 216-A-42 Diversion Basin (A42B3) to 216-B-3 Pond, 200 East Area, in 1993. (H104)

\begin{tabular}{|c|c|c|}
\hline (1) Radirnuclide & Release & 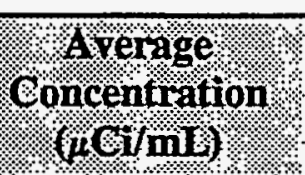 \\
\hline${ }^{3} \mathrm{H}$ & $5.8 \mathrm{E}-03$ & $1.1 \mathrm{E}-06$ \\
\hline${ }^{90} \mathrm{SI}$ & $\overline{\mathrm{ND}}$ & $<3.2 \mathrm{E}-08$ \\
\hline${ }^{137} \mathrm{Cs}$ & $6.0 \mathrm{E}-04$ & $1.1 \mathrm{E}-07$ \\
\hline${ }^{147} \mathrm{Pm}$ & ND & $<9.0 \mathrm{E}-07$ \\
\hline $239,240 \mathrm{Pu}$ & $1.3 \mathrm{E}-04$ & $2.3 \mathrm{E}-08$ \\
\hline$\overline{241} \mathrm{Am}$ & ND & $<4.3 \mathrm{E}-08$ \\
\hline Total $\alpha$ & $5.9 \mathrm{E}-05$ & $1.1 \mathrm{E}-08$ \\
\hline Total B & $1.1 \mathrm{E}-03$ & $2.1 \mathrm{E}-07$ \\
\hline Flow & \multicolumn{2}{|c|}{$5.4 \mathrm{E}+06 \mathrm{~L}(1.4 \mathrm{E}+06 \mathrm{gal})$} \\
\hline
\end{tabular}

$\mathrm{ND}=$ none detected

Table 3-9. Radionuclides in 242-A Evaporator Cooling Water Discharges (ACW) to 216-B-3 Pond, 200 East Area, in 1993. (H108)

\begin{tabular}{|c|c|c|}
\hline \% & (. Release & $\begin{array}{l}\text { A rerage } \\
\text { Concentration } \\
\text { (aeijmu }\end{array}$ \\
\hline${ }^{3} \mathrm{H}$ & $3.4 \mathrm{E}-00$ & $4.9 \mathrm{E}-06$ \\
\hline${ }^{90} \mathrm{Sr}$ & $1.1 \mathrm{E}-02$ & $1.6 \mathrm{E}-08$ \\
\hline$\overline{137} \mathrm{Cs}$ & ND & $<4.8 \mathrm{E}-08$ \\
\hline $239,240 \mathrm{Pu}$ & ND & $<1.4 \mathrm{E}-08$ \\
\hline${ }^{241} \mathrm{Am}$ & ND & $<2.7 \mathrm{E}-08$ \\
\hline Total $\alpha$ & $2.0 \mathrm{E}-03$ & $2.9 \mathrm{E}-09$ \\
\hline Total B & $6.8 \mathrm{E}-03$ & $9.7 \mathrm{E}-09$ \\
\hline Flow & \multicolumn{2}{|c|}{$7.0 \mathrm{E}+08 \mathrm{~L}(1.9 \mathrm{E}+08 \mathrm{gal})$} \\
\hline
\end{tabular}

$\mathrm{ND}=$ none detected 
Table 3-10. Radionuclides in 242-A EvaporatorCrystallizer Steam Condensate Discharges (ASC) to 216-B-3 Pond, 200 East Area, in 1993. (H110)

\begin{tabular}{|c|c|c|}
\hline Radionuclide & $\begin{array}{l}\text { Release } \\
\text { (Ci) }\end{array}$ & $\begin{array}{l}\text { A verage } \\
\text { Concentration } \\
(\mu \mathrm{ei} i \mathrm{mi})\end{array}$ \\
\hline${ }^{3} \mathrm{H}$ & ND & $<4.5 \mathrm{E}-07$ \\
\hline${ }^{90} \mathrm{Sr}$ & $8.3 \mathrm{E}-05$ & $1.2 \mathrm{E}-08$ \\
\hline$\overline{137} \mathrm{Cs}$ & $\overline{\mathrm{ND}}$ & $<4.3 \mathrm{E}-08$ \\
\hline$\overline{\mathrm{U}, \text { total }}$ & $1.4 \mathrm{E}-05$ & $2.1 \mathrm{E}-09$ \\
\hline${ }^{239,240} \mathrm{Pu}$ & $\overline{\mathrm{ND}}$ & $<1.4 \mathrm{E}-08$ \\
\hline$\overline{241} \mathrm{Am}$ & ND & $<2.9 \mathrm{E}-08$ \\
\hline Total $\alpha$ & $2.3 \mathrm{E}-05$ & $3.4 \mathrm{E}-09$ \\
\hline Total B & $2.2 \mathrm{E}-04$ & $3.4 \mathrm{E}-08$ \\
\hline Flow & \multicolumn{2}{|c|}{$6.7 \mathrm{E}+06 \mathrm{~L}(1.8 \mathrm{E}+06 \mathrm{gal})$} \\
\hline
\end{tabular}

$\mathrm{ND}=$ none detected

Table 3-11. Radionuclides in 241-A Tank Farm Cooling Water Discharges (CA8) to 216-B-3 Pond, 200 East Area, in 1993. (H115)

\begin{tabular}{|c|c|c|}
\hline Radionuclide & $\begin{array}{l}\text { Release } \\
(\mathrm{Ci})\end{array}$ & $\begin{array}{l}\text { A rerage } \\
\text { Concentration } \\
(\mu \mathrm{c} \text { ilimi }\end{array}$ \\
\hline${ }^{90} \mathrm{Sr}$ & $1.2 \mathrm{E}-02$ & $1.4 \mathrm{E}-08$ \\
\hline.${ }^{137} \mathrm{Cs}$ & ND & $<4.3 \mathrm{E}-08$ \\
\hline $239,240 \mathrm{Pu}$ & $\overline{\mathrm{ND}}$ & $<1.8 \mathrm{E}-08$ \\
\hline${ }^{241} \mathrm{Am}$ & ND & $<2.8 \mathrm{E}-08$ \\
\hline Total $\alpha$ & $7.2 \mathrm{E}-04$ & $8.2 E-10$ \\
\hline Total B & $1.8 \mathrm{E}-02$ & $2.0 \mathrm{E}-08$ \\
\hline Flow & \multicolumn{2}{|c|}{$8.8 \mathrm{E}+08 \mathrm{~L}(2.3 \mathrm{E}+08 \mathrm{gal})$} \\
\hline
\end{tabular}

$\mathrm{ND}=$ none detected 
Table 3-12. Radionuclides in 244-AR Vault Cooling Water Discharges (CAR) to 216-B-3 Pond, 200 East Area, in 1993. (H116)

\begin{tabular}{|c|c|c|}
\hline 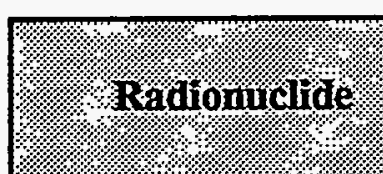 & 1. Release, & 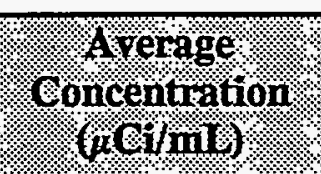 \\
\hline${ }^{90} \mathrm{Sr}$ & $1.2 \mathrm{E}-03$ & $1.3 \mathrm{E}-08$ \\
\hline$\overline{137} \mathrm{Cs}$ & ND & $<4.4 \mathrm{E}-08$ \\
\hline $239,240 \mathrm{Pu}$ & $2.6 \mathrm{E}-03$ & $2.9 \mathrm{E}-08$ \\
\hline${ }^{241} \mathrm{Am}$ & ND & $<2.9 \mathrm{E}-08$ \\
\hline Total $\alpha$ & $1.2 \mathrm{E}-04$ & $1.3 \mathrm{E}-09$ \\
\hline Total B & $5.1 \mathrm{E}-04$ & $5.6 \mathrm{E}-09$ \\
\hline Flow & \multicolumn{2}{|c|}{$9.0 \mathrm{E}+07 \mathrm{~L}(2.4 \mathrm{E}+07)$} \\
\hline
\end{tabular}

$\mathrm{ND}=$ none detected

Table 3-13. Radionuclides in B Plant Cooling Water (CBC) Discharges to 216-B-3 Pond, 200 East Area, in 1993. (H117)

\begin{tabular}{|c|c|c|}
\hline 4 & Release & $\begin{array}{c}\text { A Arerage } \\
\text { Concentration } \\
\text { (eifimi) }\end{array}$ \\
\hline${ }^{90} \mathrm{Sr}$ & $3.3 \mathrm{E}-02$ & $1.3 \mathrm{E}-08$ \\
\hline${ }^{137} \mathrm{Cs}$ & ND & $<4.1 \mathrm{E}-08$ \\
\hline $239,240 \mathrm{Pu}$ & ND & $<2.2 \mathrm{E}-08$ \\
\hline${ }^{241} \mathrm{Am}$ & ND & $<2.8 \mathrm{E}-08$ \\
\hline Total $\alpha$ & $2.3 \mathrm{E}-03$ & $9.2 \mathrm{E}-10$ \\
\hline Total B & $2.0 \mathrm{E}-02$ & $8.0 \mathrm{E}-09$ \\
\hline Flow & \multicolumn{2}{|c|}{$2.5 \mathrm{E}+09 \mathrm{~L}(6.7 \mathrm{E}+08 \mathrm{gal})$} \\
\hline
\end{tabular}

$\mathrm{ND}=$ none detected 
Table 3-14. Radionuclides in Plutonium-Uranium Extraction Plant Chemical Sewer Discharges (CSL) to 216-B-3 Pond, 200 East Area, in 1993. (H118)

\begin{tabular}{|c|c|c|}
\hline 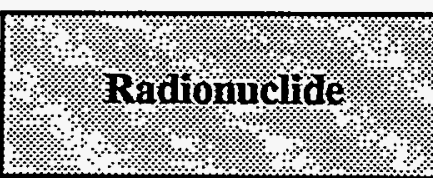 & $\left(\begin{array}{l}\text { Release } \\
(\mathrm{Ci})\end{array}\right.$ & $\begin{array}{l}\text { A yerage } \\
\text { Concentration } \\
(\text { icilmilit })\end{array}$ \\
\hline${ }^{3} \mathrm{H}$ & $4.1 \mathrm{E}-01$ & $7.4 \mathrm{E}-07$ \\
\hline${ }^{90} \mathrm{Sr}$ & $6.8 \mathrm{E}-03$ & $1.2 \mathrm{E}-08$ \\
\hline 137 Cs & ND & $<4.1 \mathrm{E}-08$ \\
\hline${ }^{147} \mathrm{Pm}$ & $\overline{\mathrm{ND}}$ & $<9.0 \mathrm{E}-07$ \\
\hline${ }^{239,240} \mathrm{Pu}$ & $1.2 \mathrm{E}-02$ & $2.2 \mathrm{E}-08$ \\
\hline${ }^{241} \mathrm{Am}$ & ND & $<2.8 \mathrm{E}-08$ \\
\hline Total $\alpha$ & $9.7 \mathrm{E}-04$ & $1.8 \mathrm{E}-09$ \\
\hline Total B & $5.2 \mathrm{E}-03$ & $9.3 \mathrm{E}-09$ \\
\hline Flow & \multicolumn{2}{|c|}{$5.5 \mathrm{E}+08 \mathrm{~L}(1.5 \mathrm{E}+08 \mathrm{gal})$} \\
\hline
\end{tabular}

$\mathrm{ND}=$ none detected

Table 3-15. Radionuclides in 200 West Area Laundry Discharges (LWC) to 216-W-LC Crib, 200 West Area, in 1993. (H120)

\begin{tabular}{|c|c|c|}
\hline Radionuclide & $\begin{array}{l}\text { Release } \\
\text { (Ci) }\end{array}$ & $\begin{array}{l}\text { aiverage } \\
\text { Concentration } \\
(1 \mathrm{Ci} / \mathrm{ml})\end{array}$ \\
\hline${ }^{90} \mathrm{Sr}$ & $8.0 \mathrm{E}-04$ & $3.0 \mathrm{E}-08$ \\
\hline$\overline{129} \mathrm{I}$ & $\mathbf{N D}$ & $<6.7 \mathrm{E}-08$ \\
\hline${ }^{137} \mathrm{Cs}$ & $1.2 \mathrm{E}-03$ & $4.5 \mathrm{E}-08$ \\
\hline $\mathrm{U}$, total & $2.3 \mathrm{E}-04$ & $8.8 \mathrm{E}-09$ \\
\hline${ }^{239,240} \mathrm{Pu}$ & $\mathrm{ND}$ & $<1.1 \mathrm{E}-08$ \\
\hline${ }^{241} \mathrm{Am}$ & ND & $<1.7 \mathrm{E}-08$ \\
\hline Total $\alpha$ & $6.5 \mathrm{E}-04$ & $2.5 \mathrm{E}-08$ \\
\hline Total B & $3.0 \mathrm{E}-03$ & $1.1 \mathrm{E}-07$ \\
\hline Flow & \multicolumn{2}{|c|}{$2.6 \mathrm{E}+07 \mathrm{~L}(7.0 \mathrm{E}+06 \mathrm{gal})$} \\
\hline
\end{tabular}

$\mathrm{ND}=$ none detected 
Table 3-16. Radionuclides in Uranium-Oxide Plant Process Condensate Discharges (U-17) to 216-U-17 Crib, 200 West Area, in 1993. (H126)

\begin{tabular}{|c|c|c|}
\hline ? & (1. Release & $\begin{array}{l}\text { A verage } \\
\text { Concentration } \\
\text { (jeimini })\end{array}$ \\
\hline${ }^{3} \mathrm{H}$ & $1.1 \mathrm{E}+01$ & $4.7 \mathrm{E}-03$ \\
\hline${ }^{99} \mathrm{Tc}$ & $8.5 \mathrm{E}-04$ & $3.6 \mathrm{E}-07$ \\
\hline $\mathrm{U}$, total & $4.8 \mathrm{E}-04$ & $2.1 \mathrm{E}-07$ \\
\hline $239,240 \mathrm{Pu}$ & $\mathrm{ND}$ & $<1.4 \mathrm{E}-08$ \\
\hline${ }^{241} \mathrm{Am}$ & ND & $<2.4 \mathrm{E}-08$ \\
\hline Total $\alpha$ & $2.4 \mathrm{E}-04$ & $1.0 \mathrm{E}-07$ \\
\hline Total B & $6.2 \mathrm{E}-03$ & $2.6 \mathrm{E}-06$ \\
\hline Flow & \multicolumn{2}{|c|}{$2.4 \mathrm{E}+06 \mathrm{~L}(6.2 \mathrm{E}+05 \mathrm{gal})$} \\
\hline
\end{tabular}

$\mathrm{ND}=$ none detected

Table 3-17. Radioactivity Discharged via 300 Area Process Sewer System in 1993.

\begin{tabular}{|c|c|c|}
\hline Radionucliade & $1 \%$ Release & 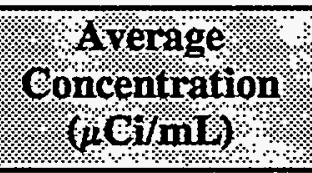 \\
\hline Total $\alpha$ & $5.2 \mathrm{E}-03$ & $1.2 \mathrm{E}-08$ \\
\hline Total $\beta$ & $2.2 \mathrm{E}-02$ & $5.1 \mathrm{E}-08$ \\
\hline Flow & \multicolumn{2}{|c|}{$4.2 \mathrm{E}+08 \mathrm{~L}(1.1 \mathrm{E}+08 \mathrm{gal})$} \\
\hline
\end{tabular}


Table 3-18. National Pollutant Discharge Elimination System Discharge Points.

\begin{tabular}{|l|l|}
\hline Designation & \\
\hline $003^{*}$ & $181-\mathrm{KE}$ Inlet Screen Backwash \\
\hline 004 & $1908-\mathrm{K}$ Outfall \\
\hline $005^{*}$ & $182-\mathrm{N}$ Tank Farm Overflow (36-in. raw water return) \\
\hline $006^{*}$ & $182-\mathrm{N}$ Drain System (42-in. raw water return) \\
\hline $007^{*}$ & $181-\mathrm{N}$ Inlet Screen Backwash \\
\hline 009 & $102-$-in. Outfall (raw water return) \\
\hline N Springs & $100-\mathrm{N}$ Riverbank Springs \\
\hline
\end{tabular}

*did not discharge any effluent during 1993

Table 3-19. Summary of National Pollutant Discharge Elimination System Data for 1993.

\begin{tabular}{|l|l|c|c|c|c|}
\hline Sample Parameter & (n) & 003 & 004 & 0.09 & N Springs \\
\hline Flow (E+06 gal/d) & Max. & $*$ & 2.2 & 0.04 & $*$ \\
& Avg. & $*$ & 0.6 & 0.03 & $*$ \\
\hline Temperature ( ${ }^{\circ} \mathrm{F}$ ) & Max. & $*$ & 74 & 68 & 77 \\
& Avg. & $*$ & & & 63 \\
\hline pH & Max. & $*$ & 8.1 & 8.3 & 7.8 \\
& Min. & $*$ & 7.2 & 7.1 & 7.2 \\
\hline Total Suspended Solids & Max. & $*$ & 5.0 & $*$ & $*$ \\
(mg/L) & Avg. & $*$ & 2.5 & $*$ & $*$ \\
\hline Oil and Grease (mg/L) & Max. & $*$ & $*$ & $*$ & 7.8 \\
& Avg. & $*$ & $*$ & $*$ & 5.6 \\
\hline Iron (mg/L) & Max. & $*$ & $*$ & $*$ & 0.6 \\
& Avg. & $*$ & $*$ & $*$ & 0.2 \\
\hline Ammonia (mg/L) & Max. & $*$ & $*$ & $*$ & 0.2 \\
& Avg. & $*$ & $*$ & $*$ & $<0.2$ \\
\hline Chromium (mg/L) & Max. & $*$ & $*$ & $*$ & $<0.01$ \\
& Avg. & $*$ & $*$ & $*$ & $<0.01$ \\
\hline Chlorine (mg/L) & Max. & $*$ & 0.12 & 0.17 & $*$ \\
& Avg. & $*$ & $<0.05$ & $<0.08$ & $*$ \\
\hline
\end{tabular}

*analysis not required 
Table 3-20. Sanitary Sewage Discharged, by Area, in 1992.

\begin{tabular}{|c|c|}
\hline Area & Discharge (G/9r [gal/yr] \\
\hline $100-\mathrm{B}$ & $1.7 \mathrm{E}+05(4.4 \mathrm{E}+04 \mathrm{gal})$ \\
\hline $100-\mathrm{D}$ & $1.0 \mathrm{E}+05(2.6 \mathrm{E}+04 \mathrm{gal})$ \\
\hline $100-\mathrm{H}$ & $1.7 \mathrm{E}+05(4.4 \mathrm{E}+04 \mathrm{gal})$ \\
\hline $100-\mathrm{K}$ & $4.3 \mathrm{E}+06(1.1 \mathrm{E}+06 \mathrm{gal})$ \\
\hline $100-\mathrm{N}$ & $2.6 \mathrm{E}+07^{2}(6.9 \mathrm{E}+06 \mathrm{gal})$ \\
\hline 200 East & $1.9 \mathrm{E}+07(4.9 \mathrm{E}+06 \mathrm{gal})$ \\
\hline 200 West & $6.7 \mathrm{E}+07(1.7 \mathrm{E}+07 \mathrm{gal})$ \\
\hline 300 & $3.3 \mathrm{E}+08(8.6 \mathrm{E}+07 \mathrm{gal})$ \\
\hline 400 & $2.4 \mathrm{E}+07(6.3 \mathrm{E}+06 \mathrm{gal})$ \\
\hline
\end{tabular}

' Approximately $1.4 \mathrm{E}+07 \mathrm{~L}$ of this total consists of septic system sanitary waste transported from the 200 Areas. 
WHC-EP-0527-3

This page is intentionally left blank. 
WHC-EP-0527-3

\subsection{HAZARDOUS SUBSTANCE RELEASES}

A hazardous substance release requires notification to appropriate federal, state, and local authorities when established limits are exceeded. Reportable releases of hazardous substances are classified as the following two types:

- Nonroutine releases

- Continuous, routine releases.

Information for each type of release is discussed separately below.

\subsection{NONROUTINE RELEASES}

The following listing shows the number of nonroutine releases that occurred in 1993, distinguished by the nature of the notifications. Other nonroutine releases and spills are not discussed in this section because they were less than agency reportable limits and the levels in DOE reporting criteria (EPA 1990a; DOE 1990d).

- 21 releases were reported to a regulatory agency

- 325 non-reportable releases were recorded

None of these releases resulted in any deleterious impacts to personnel or the environment.

\subsection{ROUTINE CONTINUOUS RELEASES}

Releases of hazardous substances that exceed CERCLA reportable quantities (RQ) need not be reported immediately to the National Response Center when both of the following conditions are met:

- An initial notification has been completed

- The routine releases are continuous and stable in quantity and rate.

The initial notification requirement has been satisfied concerning hazardous substances that have exceeded or have a potential to exceed an RQ. Historically only the continuous routine releases of ammonia and ammonium hydroxide have posed operational difficulties in staying beneath RQs. For 1993, releases of ammonia were in compliance, while no releases of ammonium hydroxide occurred. Information on ammonium hydroxide and the routine releases of ammonia is presented in Table 4-1. 
Table 4-1. Westinghouse Hanford Continuous Comprehensive Environmental Response, Compensation, and Liability Act of 1980 Release Summary for Calendar Year 1993."

\begin{tabular}{|c|c|c|c|c|c|}
\hline 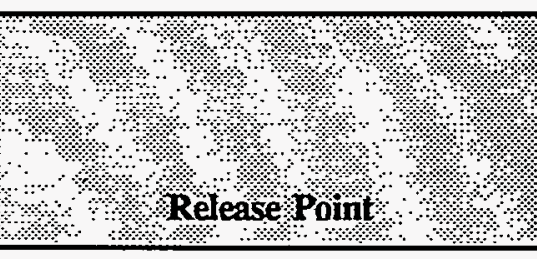 & +. & 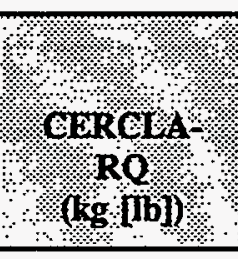 & $\begin{array}{l}\text { Number of } \\
\text { Occurrences } \\
\text { whiat } \\
\text { Roveded }\end{array}$ & 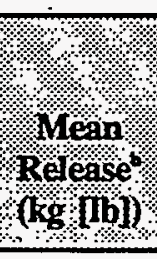 & 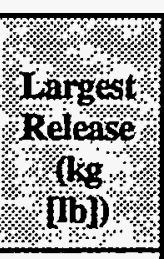 \\
\hline $\begin{array}{l}\text { 296-A-24 (PUREX Plant } \\
\text { Ammonia scrubber waste } \\
\text { concentrator exhaust) }\end{array}$ & ammonia & $\begin{array}{r}45.4 \\
(100)\end{array}$ & c & - & - \\
\hline $\begin{array}{l}\text { 296-A-40 (241-AP Tank Farm } \\
\text { primary exhaust) }\end{array}$ & ammonia & $\begin{array}{l}45.4 \\
(100)\end{array}$ & 0 & $\begin{array}{c}0.9 \\
(1.9)\end{array}$ & $\begin{array}{c}1.8 \\
(4.0)\end{array}$ \\
\hline $\begin{array}{l}\text { 296-A-27 (241-AW Tank Farm } \\
\text { primary exhaust) }\end{array}$ & ammonia & $\begin{array}{r}45.4 \\
(100)\end{array}$ & 0 & $\begin{array}{c}0.7 \\
(1.5)\end{array}$ & $\begin{array}{c}1.5 \\
(3.2)\end{array}$ \\
\hline $\begin{array}{l}\text { 296-A-22 (242-A Evaporator- } \\
\text { Crystallizer process condensate) }\end{array}$ & $\begin{array}{l}\text { ammonium } \\
\text { hydroxide }\end{array}$ & $\begin{array}{c}454 \\
(1,000)\end{array}$ & c & - & - \\
\hline $\begin{array}{l}\text { 296-A-22 (242-A Evaporator- } \\
\text { Crystallizer process condensate) }\end{array}$ & ammonia & $\begin{array}{r}45.4 \\
(100)\end{array}$ & 0 & 0 & 0 \\
\hline $\begin{array}{l}\text { 291-Z-1 (Plutonium Reclamation } \\
\text { Facility) }\end{array}$ & $\begin{array}{l}\text { carbon } \\
\text { tetrachloride }\end{array}$ & $\begin{array}{l}4.54 \\
(10)\end{array}$ & 1 & $\begin{array}{c}12.1 \\
(26.6)\end{array}$ & $\begin{array}{c}12.1 \\
(26.6)\end{array}$ \\
\hline
\end{tabular}

"All quantities are for any continuous 24-hr period.

'Mean release is the annual quantity divided by the number of operating days in calendar year 1993.

This facility or emission point did not operate in 1993.

CERCLA = Comprehensive Environmental Response, Compensation, and Liability Act of 1980

$\mathrm{RQ}=$ Reportable quantity 


\subsection{REFERENCES}

40 CFR 61, National Emission Standards for Hazardous Air Pollutants, Title 40, Code of Federal Regulations, Part 61, Subpart H, U.S. Environmental Protection Agency, Washington, D.C.

40 CFR 302, Designation, Reportable Quantities, and Notification, Title 40, Code of Federal Regulations, Part 302, U.S. Environmental Protection Agency, Washington, D.C.

BFCCAA, 1993, General Regulation 1 of the Benton-Franklin Counties Clean Air Pollution Control Authority, Benton-Franklin Counties Air Pollution Control Authority, Richland, Washington.

Clean Water Act of 1977, Public Law 95-217, 92 (Stat) 1566, 33 USC 125.

Diediker, L. P., 1993, Environmental Releases for Calendar Year 1992, WHC-EP-0527-2, Westinghouse Hanford Company, Richland, Washington.

DOE, 1990, Environmental Protection, Safety, and Health Protection Information Reporting Requirements, DOE Order 5484.1, Change 7, U.S. Department of Energy, Washington, D.C.

DOE, 1990a, General Environmental Protection Program, DOE Order 5400.1, Change 1, U.S. Department of Energy, Washington, D.C.

DOE, 1990b, Radiation Protection of the Public and the Environment, DOE Order 5400.5, U.S. Department of Energy, Washington, D.C.

DOE, 1990c, Environment, Safery, and Health Program for Department of Energy Operations, DOE Order 5480.1B, Change 4, U.S. Department of Energy, Washington, D.C.

DOE, 1990d, Occurrence Reporting and Processing for Department of Energy Operations, DOE Order 5480.1B, Change 4, U.S. Department of Energy, Washington, D.C.

DOE-RL, 1994, Radionuclide Air Emissions Report for the Hanford Site, Calendar Year 1993, DOE/RL-94-51, U.S. Department of Energy, Richland Operations, Richland, Washington.

EPA, 1990, AIRS Facility Subsystem Source Classification Codes and Emission Factor Listing for Criteria Air Pollutants, EPA 450/4-90-003, U.S. Environmental Protection Agency, Research Triangle Park, North Carolina. 


\subsection{REFERENCES (continued)}

PNL, 1994, Hanford Site Environmental Report for Calendar Year 1993, PNL-9823, Pacific Northwest Laboratory, Richland, Washington.

WAC, 173-303, Dangerous Waste Regulations, Washington Administrative Code 173-303, Olympia, Washington.

WHC, 1993, Hanford Site Radioactive Solid Waste Acceptance Criteria, WHC-EP-0063-4, Westinghouse Hanford Company, Richland, Washington.

WHC, 1994, Liquid Effluent Annual Report, WHC-ED-0798-1, Westinghouse Hanford Company, Richland, Washington. 
WHC-EP-0527-3

APPENDIX A

AIR EMISSIONS AND LIQUID EFFLUENTS

A-1 
WHC-EP-0527-3

This page intentionally left blank.

A-2 


\section{APPENDIX A}

\section{AIR EMISSIONS AND LIQUID EFFLUENTS}

\section{A.1 RADIONUCLIDE AIR EMISSIONS}

Radionuclide air emissions generally are discharged from stacks and vents. Sizes, shapes, and discharge paths of stacks vary depending on facility requirements at the time of construction. Stack discharge heights range from a few meters to $61 \mathrm{~m}(200 \mathrm{ft})$, and flow rates range from less than $2,832 \mathrm{\ell} / \mathrm{min}\left(100 \mathrm{ft}^{3} / \mathrm{min}\right)$ to more than 5.7 million $\ell / \mathrm{min}$ $\left(200,000 \mathrm{ft}^{3} / \mathrm{min}\right)$.

\section{A.1.1 Filtration}

The following methods are used to remove radionuclides from stack emissions: (1) high-efficiency particulate air (HEPA) filters, (2) charcoal absorbers, (3) sand filters, (4) water scrubbers, (5) fiberglass prefilters, and (6) deep-bed fiberglass filters. Generally, at least one stage of HEPA filtration is used as the final particulate emission removal method before emissions are discharged to the atmosphere. All final filters are required to have an efficiency of $99.95 \%$ in removing airborne particles larger than $0.3 \mu \mathrm{m}$ in diameter. Filter efficiency is routinely tested.

Past release data have shown that radionuclide concentrations in some emissions are below the lower limit of analytical detection. As such, no filtration is provided for these discharges. (See Section 2.1.1.)

\section{A.1.2 100 Areas Facilities}

The 100 Areas contain eight inactive production reactors, the $\mathrm{N}$ Reactor (now in transition to deactivation and decommissioning), and associated support facilities. Radionuclide air emission points at facilities in the 100-N Area and 100-K Area are described briefly below and shown respectively in Figures 1-2 and 1-3.

A.1.2.1 N Reactor. This dual-purpose reactor operated until 1987, producing electrical power for the regional energy system and plutonium for weapons production. Because further operation of the $\mathrm{N}$ Reactor is not anticipated, the reactor and associated facilities are now in transition to deactivation and decommissioning.

- 116-N. This stack exhausts filtered air from the 105-N Reactor Building. Emission monitoring consists of a record sampler.

- 109-N-Z-I. This vent did not operate in 1993. When the vent was operational, unfiltered air was released through it from 109-N Steam Generator Cells 1 
through 5. Emission monitoring consisted of a record sampler and a grossgamma-energy monitor.

- 109-N-C-6. This vent did not operate in 1993. When it did, unfiltered air was released through it from Steam Generator Cell 6 in the 109-N Building. Emission monitoring consisted of a record sampler and a gross-gamma-energy monitor.

- 107-N. This vent exhausts filtered air from the 107-N Basin Recirculation Building. Emission monitoring consists of a record sampler. This emission point is not registered with WDOH.

- 105-N Basin Transfer Area. This vent exhausts unfiltered air from the N Basin and the transfer area for rail-transported well cars. Emissions are continuously monitored by a record sampler. This emission point is not registered with WDOH.

- 105-N 14-Ft. Decontamination Room. This stack exhausts filtered air from a small room in which radioactively contaminated tools are decontaminated. Emissions are continuously monitored by a record sampler. This emission point is not registered with WDOH.

A.1.2.2 100-K East and West Areas. These areas contain two retired reactors awaiting decommissioning, two water-filled storage basins holding irradiated nuclear fuel, and radiological analysis laboratories.

- 105-KE. This vent exhausts unfiltered air from the fuel storage basin in the 105-KE Building. Emission monitoring consists of a record sampler.

- 105-KW. This vent exhausts unfiltered air from the fuel storage basin in the 105-KW Building. Emission monitoring consists of a record sampler.

- 1706-KE. This stack exhausts filtered air from the 1706-KE Laboratory. Emission monitoring consists of a record sampler.

- 1706-KER. This vent exhausts filtered air from the basement area (approximately 27 feet [8.2 m] below grade) of the 1706-KE Laboratory. Emission monitoring consists of a record sampler.

\section{A.1.3 200 Areas Facilities}

The 200 Areas contain facilities for chemical separations, processing, and waste handling and disposal. The PUREX Plant and related tank farm and evaporator facilities discharge volatile forms of radionuclides, specifically ${ }^{106} \mathrm{Ru},{ }^{125} \mathrm{Sb}$, and ${ }^{129} \mathrm{I}$. When operating, 
the PUREX Plant discharged ${ }^{85} \mathrm{Kr}$, but it has not operated fully since December 1989 . No activities conducted in 1993 resulted in a discharge of ${ }^{85} \mathrm{Kr}$.

A.1.3.1 PUREX Plant. The PUREX Plant is a nuclear-fuel processing plant constructed in 1956 and operated until 1972, when it was placed on standby status. Later, needed modifications were made and the PUREX Plant resumed operations in November 1983, processing a backlog of irradiated fuel from $\mathbf{N}$ Reactor. The plant was placed in standby following a stabilization run that ended in March 1990.

- 291-A-1. This stack exhausts filtered air from canyon ventilation (Cells $A$ to $\mathbf{M})$, vessel and condenser vents, and the dissolver offgas system. Emission monitoring consists of a record sampler, a silver zeolite cartridge, an alpha continuous air monitor (CAM), and a beta-gamma CAM.

- 296-A-1. This stack exhausts filtered air from $N$ and Q Cells, Product Removal rooms, and gloveboxes in these rooms. Emission monitoring consists of a record sampler and an alpha CAM.

- 296-A-2. This stack exhausts filtered air from the west sample gallery hoods. Emission monitoring consists of a record sampler, an alpha CAM, and a beta-gamma CAM.

- 296-A-3. This stack exhausts filtered air from hoods in the east sample gallery. Emission monitoring consists of a record sampler and a beta-gamma CAM.

- 296-A-5A and 296-A-5B. These stacks exhaust filtered air from laboratory hoods. Emission monitoring for each stack consists of a record sampler, an alpha CAM, and a beta-gamma CAM.

- 296-A-6. This stack exhausts filtered room air from the east sample gallery and $\mathrm{U}$ Cell. Emission monitoring consists of a record sampler.

- 296-A-7. This stack exhausts filtered room air from the PUREX west sample gallery and $\mathbf{R}$ Cell. Emission monitoring consists of a record sampler.

- 296-A-8. This stack exhausts filtered air from the PUREX Plant Pipe and Operating gallery and the White Room. Emission monitoring consists of a record sampler.

- 296-A-10. This stack exhausts filtered air from storage tunnel No. 2. Emission - monitoring consists of a record sampler. 
- 296-A-14. This stack exhausts filtered air from the 293-A Offgas Treatment and Recovery Building (for nitric acid recovery). Emission monitoring consists of a record sampler.

- 296-A-24. This stack, known as the ammonia-scrubber waste-concentrator stack, did not operate in 1993. Its monitoring systems have been deactivated and its emission points sealed. When the stack did operate, filtered air was released through it from the $\mathrm{E}$ and $\mathrm{F}$ Cell vessels to reduce ammonium-nitrate loading on the 291-A-1 filters. Emission monitoring consisted of a record sampler, two silver-zeolite cartridge samplers for volatile radionuclides, an alpha CAM, a beta-gamma CAM, and an ammonia monitor.

- 296-A-33, -34, -35, -36, -37, and -38. All of these exhausters are now deactivated and will be deregistered with WDOH. Exhausters 296-A-33, -35, and -37 were deactivated before 1991, and exhausters $296-\mathrm{A}-34$ and -36 by the end of 1991. Exhauster 296-A-38 did not operate in 1993. The exhauster emissions were continuously monitored by record samplers and beta-gamma CAMs.

A.1.3.2 B Plant and Waste Encapsulation Storage Facility. This facility contains two major operating system areas, B Plant and the Waste Encapsulation Storage Facility (WESF). B Plant was designed and used to remove ${ }^{137} \mathrm{Cs}$ and ${ }^{90} \mathrm{Sr}$ from high-level liquid waste. At WESF, the ${ }^{137} \mathrm{Cs}$ and ${ }^{90} \mathrm{Sr}$ were converted to solid strontium fluoride and cesium chloride, doubly encapsulated, and placed in water-filled storage basins. WESF now is used to store radioactive inventories, and contains many radioactive strontium and cesium capsules. The 221-B Building contains radioactive contamination remaining from previous production campaigns that must be properly managed.

- 291-B-1. This is the B Plant main stack, which exhausts filtered air from the B Plant canyon, the process vessel ventilation system number 1 , and the 212-B and 224-B Buildings. Emission monitoring consists of a record sampler, a backup record sampler, and a beta-gamma CAM.

- 296-B-5. This stack exhausts filtered air from the 221-BB Building, which houses the B Plant process condensate and B Plant steam condensate receiver tanks. Emission monitoring consists of a record sampler and a beta-gamma CAM.

- 296-B-10. This stack exhausts filtered air from the 225-B Building, which is also known as WESF. Emission monitoring consists of a record sampler and a beta-gamma CAM. 
- 296-B-13. This stack exhausts filtered air from the 221-BF Condensate Effluent Discharge Facility, which houses process condensate retention ventilation. Emission monitoring consists of a record sampler and a beta-gamma CAM.

- 296-B-14. This stack exhausts filtered air from the 221-B Building and the vessel ventilation system No. 2. Emission monitoring consists of a record sampler and a process sampler. This stack did not operate in 1993.

A.1.3.3 AR and CR Vaults. The AR and CR Vaults are retention and treatment facilities for high-level radioactive liquid wastes.

- 296-A-12. This stack exhausts filtered air from the 244-AR Vault vessel ventilation system. Emission monitoring consists of a record sampler and a beta-gamma CAM. This stack did not operate in 1993.

- 296-A-13. This stack exhausts filtered air from the 244-AR Vault canyon and cells. Emission monitoring consists of a record sampler and a beta-gamma CAM.

- 296-C-5. This stack exhausts filtered air from the 244-CR Vault Cell and vessel ventilation. Emission monitoring consists of a record sampler and a beta-gamma CAM.

A.1.3.4 200 East Area Tank Farms (excluding the Evaporator). Radioactive waste stored in tank farms consists of sludge and salt cake in single-shell tanks (SSTs) and slurry in double-shell tanks (DSTs).

- 296-A-17. This stack exhausts filtered, noncondensable vapors from waste storage tanks in the 241-AY and -AZ Tank Farms. Emission monitoring consists of a record sampler, two silver-zeolite cartridge samplers for volatile radionuclides, and a beta-gamma CAM.

- 296-A-18. This stack exhausts filtered air from the 241-AY-101 tank annulus. Emission monitoring consists of a record sampler. This stack has been inactive since September 1991.

- 296-A-19. When this stack was operational, filtered air was released through it from the 241-AY-102 tank annulus. Emission monitoring consisted of a record sampler. This stack did not operate in 1993.

- 296-A-20. 241-AZ Tank Annuli Exhaust. This stack exhausts filtered air from the 241-AZ-101 and -102 tank annuli. Emission monitoring consists of a record sampler. 
- 296-A-25. This stack exhausts filtered air from the catch tank at the 244-A lift station. Emission monitoring consists of a record sampler and a beta-gamma CAM. This stack did not operate in 1993.

- 296-A-26. This stack exhausts filtered air from the waste unloading room and sump tank at the 204-AR tank. Emission monitoring consists of a record sampler and a beta-gamma CAM.

- 296-A-27. This stack exhausts filtered air from all 241-AW tanks. Emission monitoring consists of a record sampler, two silver-zeolite cartridge samplers for volatile radionuclides, and a beta-gamma CAM.

- 296-A-28. This stack exhausts filtered air from all tank annuli in the 241-AW tank farm. Emission monitoring consists of a record sampler and a beta-gamma CAM.

- 296-A-29. This stack exhausts filtered air from all 241-AN tanks. Emission monitoring consists of a record sampler and a beta-gamma CAM.

- 296-A-30. This stack exhausts filtered air from all tank annuli in the 241-AN tank farm. Emission monitoring consists of a record sampler and a beta-gamma CAM.

- 296-A-40. This stack exhausts filtered air from all 241-AP tanks. Emission monitoring consists of a record sampler, two silver-zeolite cartridge samplers for volatile radionuclides, and a beta-gamma CAM.

- 296-A-41. This stack exhausts filtered air from all tank annuli in the 241-AP tank farm. Emission monitoring consists of a record sampler and a beta-gamma CAM.

- 296-B-28. This stack exhausts filtered air from the 244-BX salt well receiver tanks and annulus. Emission monitoring consists of a record sampler, a beta-gamma CAM for the tank exhaust, and a beta-gamma CAM for the annulus exhaust.

- 296-P-16. A portable exhauster emits filtered air via this stack from the 241-C-104, -105, and -106 tanks. Emission monitoring consists of a record sampler and a beta-gamma CAM.

- 296-P-17. This portable exhauster emits filtered air from the 241-A-104, -105, and -106 tanks. Emission monitoring consists of a record sampler and a beta-gamma CAM. This stack did not operate in 1993. 
A.1.3.5 200 East Area Evaporator. The evaporator did not process waste in 1993. The evaporator is used to remove most of the water from high-level radioactive liquid waste, leaving a slurry that is pumped back to tank farms.

- 296-A-21. This stack exhausts filtered air from the 242-A EvaporatorCrystallizer Building. Emission monitoring consists of a record sampler, an alpha CAM, and a beta-gamma CAM.

- 296-A-22. This stack exhausts filtered air from the 242-A EvaporatorCrystallizer vessel-ventilation system. Emission monitoring consists of a record sampler, two silver-zeolite cartridge samplers for volatile radionuclides, an alpha CAM, and a beta-gamma CAM.

A.1.3.6 Grout Treatment Facility. This facility solidified low-level liquid waste for disposal in underground vaults. The facility was placed in cold standby in 1993.

- 296-G-1. This stack did not operate in 1993. When it operated, this stack exhausted filtered air from the GTF Air Filtration Module. Emission monitoring consisted of a record sampler and a beta-gamma CAM.

A.1.3.7 209-E Critical Mass Laboratory. The building is no longer in operation. Formerly it was used for testing critical mass configurations.

- 296-P-31 (formerly 209-E). This stack exhausts filtered building ventilation air. The exhaust is sampled for particulate alpha and beta radioactivity.

A.1.3.8 S Plant. S Plant is another name for the Reduction-Oxidation Plant (REDOX), which is the 202-S building. The REDOX Plant was operated as a fuel-reprocessing facility until 1967 when it was shut down.

- 291-S-1. The REDOX main stack exhausts filtered air from the REDOX canyon, vessel ventilation, and treated dissolver offgas system. Emission monitoring consists of a record sampler and a beta-gamma CAM.

- 296-S-2. This stack exhausts filtered air from REDOX north and south sample galleries, hoods, and product removal cage. Emission monitoring consists of a record sampler.

- 296-S-4. This stack exhausts filtered air from the REDOX decontamination room and regulated shop and unfiltered air from the regulated tool room, low-level decontamination sink, and special work permit lobby. Emission monitoring consists of a record sampler. 
- 296-S-6. This stack exhausts air from the REDOX Plant, the silo gallery, an organic feed tank, and a sample elevator. Emission monitoring consists of a record sampler.

- 296-S-7W. This stack exhausts filtered air from the 233-S REDOX Product building, the REDOX plutonium-processing greenhouse, and process vessel ventilation and loadout area. Emission monitoring consists of a record sampler and an alpha CAM. A duplicate exhaust fan and record sampler is designated as 296-S-7E. Exhaust point 296-S-7E has not operated in several years.

A.1.3.9 T Plant. T Plant is one of the original fuel reprocessing facilities. The last fuel reprocessed there was in 1956. Now the 221-T Building is used for decontaminating and repairing equipment and the 224-T Building for storing and assaying waste suspected of containing transuranic elements (TRU).

- 291-T-1. This stack exhausts filtered air from 221-T canyon and process ventilation. Emission monitoring consists of a record sampler, a beta-gamma CAM, and an alpha CAM.

- 296-T-13. This stack exhausts filtered air from the 221-T Building and canyon ventilation. Emission monitoring consists of a record sampler and a beta-gamma CAM.

A.1.3.10 TRUSAF. The 224-T Building was originally used to purify plutonium nitrate by the lanthanum fluoride process. After phase-out of the bismuth phosphate plants, the lanthanum fluoride process was no longer needed and the facility remained inactive until the early 1970's. At that time, the 224-T Building was modified for storage of plutonium scrap in liquid and solid forms. In 1984 the facility was selected to house the transuranic waste storage and assay (TRUSAF) operation. The TRUSAF operation consists of analysis of transuranic waste to verify compliance with the Waste Isolation Pilot Plant (WIPP) waste acceptance criteria. Those containers meeting WIPP acceptance criteria are stored at 224-T pending shipment to the WIPP.

- 296-T-11. This stack exhausts filtered air from the 224-T Building ventilation through the west part of the plenum. Emission monitoring consists of a record sampler.

- 296-T-12. This stack exhausts filtered air from the 224-T Building ventilation through the east part of the plenum. Emission monitoring consists of a record sampler.

A.1.3.11 U Plant. U Plant was constructed as a fuel reprocessing plant but was never used for that purpose. Instead, it was used to recover uranium from bismuth-phosphate waste and high-level radioactive wastes from tank farms. Now, however, it is a shut-down, retired facility with a few offices and shops still in use. Located adjacent to U Plant is the 
Uranium-Oxide $\left(\mathrm{UO}_{3}\right)$ Plant, which formerly produced uranium trioxide from PUREX Plant solutions. In 1972 the $\mathrm{UO}_{3}$ Plant was shut down. Later, it was refurbished to process uranyl nitrate hexahydrate from the PUREX Plant, and was restarted in March 1984. The final $\mathrm{UO}_{3}$ clean-out campaign was conducted in 1993.

- 291-U-1. This stack exhausts filtered air from U Plant and 221-U canyon ventilation. Emission monitoring consists of a record sampler and a beta-gamma CAM.

- 296-U-2. This stack exhausts filtered air from the powder-handling system in the 224-UA Building of the $\mathrm{UO}_{3}$ Plant. Emission monitoring consists of a record sampler.

- 296-U-4. This $\mathrm{UO}_{3}$ Plant stack exhausts unfiltered air from the process offgas system (vessel vent, concentrator, and calciner) on the 224-U Building. Emission monitoring consists of a record sampler.

- 296-U-13. This stack exhausts filtered air from the powder loadout hood. Emission monitoring consists of a record sampler and beta-gamma CAM.

A.1.3.12 Plutonium Finishing Plant. PFP conducted a personnel training run without feed material in 1993. Historically, PFP, located in the 200 West Area, recovered plutonium in the form of plutonium nitrate from plutonium scrap. Plutonium metal was also produced from recovered nitrate and plutonium nitrate received from the PUREX Plant. This facility now is used to stabilize and store plutonium. The reduction process stabilizes the plutonium into the state best suited for long-term storage. PFP's current mission is to stabilize the existing inventory of plutonium waste.

- 291-Z-1. This stack exhausts filtered air from the 234-5Z, 236-Z, and 242-Z Buildings. Emission monitoring consists of a record sampler and an alpha CAM.

- 296-Z-3. This stack exhausts filtered air from $241-Z$ vault sump and vessel ventilation. Emission monitoring consists of a record sampler and an alpha CAM.

- 296-Z-5. This stack exhausts filtered air from 2736-ZB, the shipping and receiving building. Emission monitoring consists of a record sampler and an alpha CAM.

- 296-Z-6. This stack exhausts filtered air from the 2736-ZA Building and its plutonium storage vault ventilation system. Emission monitoring consists of a record sampler and two alpha CAMs. One alpha CAM is located on each of the two ducts feeding into this stack. 
- 296-Z-14. This stack exhausts filtered air from the 232-Z Incinerator Building. Emission monitoring consists of a record sampler and two alpha CAMs.

A.1.3.13 200 West Area Tank Farms (Excluding Evaporators). Radioactive waste stored in tank farms consists of sludge and salt cake in SSTs and liquid and slurry in DSTs.

- 296-P-22. This stack exhausts filtered air from annuli in the 241-SY-101, -102, and -103 tanks. Emission monitoring consists of a record sampler and a beta-gamma CAM.

- 296-P-23. This stack exhausts filtered air from the 241-SY-101, -102 , and -103 tanks. Emission monitoring consists of a record sampler and a beta-gamma CAM.

- 296-S-15. This stack exhausts filtered air from tanks 241-SX-107 through -112 and 241-SX-114. Emission monitoring consists of a record sampler and a beta-gamma CAM.

- 296-S-22. This stack exhausts filtered air from the 244-S salt well receiver tank and annulus. Emission monitoring consists of a record sampler, an alpha CAM, and a beta-gamma CAM.

- 296-T-18. This stack exhausts filtered air from the 244-TX salt well receiver tank and annulus. Emission monitoring consists of a record sampler, an alpha CAM, and a beta-gamma CAM.

A.1.3.14 200 West Area Evaporators. The 242-T Evaporator-Crystallizer is shut down, and therefore did not operate in 1993. The 242-S Evaporator-Crystallizer also did not operate in 1993, and is on standby. The evaporators are designed to remove most of the water from radioactive liquid waste; the resulting slurry is then routed to tank farms for storage.

- 296-S-18. This stack exhausts filtered air from the 242-S Evaporator-Crystallizer Building. Emission monitoring consists of a record sampler, an alpha CAM, and a beta-gamma CAM.

- 296-T-17. This stack exhausts filtered air from the 242-T EvaporatorCrystallizer and cold-cell ventilation. Emission monitoring consists of a record sampler, an alpha CAM, and a beta-gamma CAM.

A.1.3.15 2724-W Laundry. This is the Protective Equipment and Decontamination Facility, also known as the 2724-W Laundry, located in the 200 West Area. Radioactively contaminated protective clothing and equipment used by Hanford Site contractors was laundered at this facility. This facility was shut down in 1993. 
- 296-W-1. This stack exhausts air from clothes dryers and a washroom. The air is scrubbed with a water wash before exhausting through the stack. Emission monitoring consists of a record sampler.

A.1.3.16 222-S Analytical Laboratories. The 222-S Analytical Laboratories provide chemical and radiochemical analytical support for Westinghouse Hanford, including environmental and effluent sample analysis, research and development, and tank farm waste characterization.

- 296-S-16. This stack exhausts filtered air from the 219-S Building vault and waste tanks. Emission monitoring consists of a record sampler.

- 296-S-21. This stack exhausts filtered air from 222-S Laboratory hoods, gloveboxes, hot-cells, and room ventilation system. Emission monitoring consists of a record sampler, an alpha CAM, and a beta-gamma CAM.

A.1.3.17 Waste Compactor. The facility compacts the volume of radioactive solid waste.

- 296-W-3. This exhaust discharges filtered air from the 213-W compactor. Emission monitoring consists of a record sampler and a beta-gamma CAM.

\section{A.1.4 300 Area Facilities}

The 300 Area consists primarily of laboratories, research facilities, and a steam plant. Emission points in the 300 Area are shown in Figure 1-6.

A.1.4.1 306-E Metal Fabrication Development Building. This building is in use for materials testing and fabrication. In the past, this building housed three separate operations; (1) a large, high-bay used for nonfueled test article fabrication for FFTF, (2) nondestructive, radiography examination in a series of shielded cells, and (3) a small depleted-uraniumpowder laboratory used to fabricate insulator pellets for the FFTF fuel pins.

- 306-E-ULAB. This stack exhausts filtered air from the Uranium Oxide Laboratories in rooms 158 and 159 . The laboratories are currently shut down. Emission monitoring consists of a record sampler.

A.1.4.2 308 Fuels Development Laboratory. The 308 Laboratory was used for fabricating mixed-oxide fuel pins used by FFTF. The facility houses a shutdown swimming-pool-type Test Reactor and Isotope Production reactor by General Atomics (TRIGA) that was used in neutron radiography of the completed pins. The 308 Building is in transition to shutdown.

- 308-GL-EX. This exhaust discharges filtered air from gloveboxes and hoods in the 308 Building. Emission monitoring consists of a record sampler and an alpha CAM. 
- 308-ET-EX. This exhaust discharges filtered air from those portions of the 308 Laboratory previously associated with etching and cleaning operations, discontinued several years ago. Emission monitoring consists of a record sampler and an alpha CAM.

- 308-TRIGA. This stack discharges filtered air from the reactor hall housing the TRIGA reactor. The reactor, however, is permanently shutdown and partially defueled. Emission monitoring consists of a record sampler, an alpha CAM, and a record sampler for ${ }^{131} \mathrm{I}$.

A.1.4.3 340 Complex. The 340 Complex houses the radioactive liquid waste and solid waste handling operations for the 300 Area. The 340-A Building contains six aboveground storage tanks for radioactive liquid waste. The east side of the building has a railcar loadout facility for shipping liquid waste to the 200 Areas. The west side is a storage area for radioactive waste.

- 340-NT-EX. 340 Tank and Vault Exhaust. This stack exhausts filtered air from the 340-A Building vault and waste tanks. Emission monitoring consists of a record sampler, an alpha CAM, a beta-gamma CAM, a radioiodine CAM, and a record sampler for ${ }^{131} \mathrm{I}$.

\section{A.1.5 400 Area Facilities}

The 400 Area consists of the FFTF, the Maintenance and Storage Facility (MASF), and the Fuels Materials Examination Facility. Emission points in the 400 Area are shown in Figure 1-7.

A.1.5.1 Fast Flux Test Facility. FFTF, located in the 400 Area, is a 400-megawatt thermal, sodium-cooled, low-pressure, high-temperature reactor plant. It was used for irradiation testing of breeder reactor fuels and materials.

- FFTF-RE-SB. Lower Reactor Service Building (RSB). This exhaust discharges unfiltered air from the lower level of the RSB. Emission monitoring consists of a record sampler, a beta CAM, and a noble gas monitor.

- FFTF-CB-EX. Combined exhaust. This stack exhausts normally unfiltered air from the reactor containment and gases from the argon processing system. Standby particulate filters are automatically dampered into the system if airborne radioactive particulate levels rise above certain concentrations. Emission monitoring consists of a record sampler, a continuous tritium monitor, a beta CAM, a noble gas monitor, and a record sampler for ${ }^{131} \mathrm{I}$. The tritium record sampler was eliminated on December 12, 1993.

- FFTF-HT-TR. Heat Transport System South. This exhaust discharges normally unfiltered air from portions of FFTF that are exterior to the containment. 
Standby particulate filters are automatically dampered into the system if airborne radioactive particulate levels rise above certain concentrations. Emission monitoring consists of a record sampler, a beta CAM, and a noble gas monitor.

A.1.5.2 437 MASF. The Maintenance and Storage Facility (MASF) is a multipurpose service center supporting the specialized maintenance and storage requirements of FFTF. The MASF provides the capability for sodium film removal, decontamination, repair, and storage of nonfueled components and hardware for FFTF.

- 437-MN\&ST. This exhaust discharges filtered air from the MASF. Emission monitoring consists of a record sampler and a beta CAM. 
WHC-EP-0527-3

This page is intentionally left blank. 
WHC-EP-0527-3

APPENDIX B

MONITORING PROGRAM FOR AIR EMISSIONS AND LIQUID EFFLUENTS

B-1 
WHC-EP-0527-3

This page intentionally left blank. 
WHC-EP-0527-3

\section{APPENDIX B}

\section{MONITORING PROGRAM FOR AIR EMISSIONS AND LIQUID EFFLUENTS}

The Westinghouse Hanford Company (Westinghouse Hanford) monitoring program for air emissions and liquid effluents comprises several activities designed to obtain and report high-quality sampling and analysis data to determine compliance with U.S. Department of Energy guidelines and federal, state, and local regulations.

\section{B.1 AIR EMISSION SAMPLE COLLECTION AND ANALYSIS}

A representative sample of air emissions during the collection period is obtained by extracting the sample at a velocity that is as close as practicable to the stack airflow velocity and by holding the ratio of sample volume to total stack discharge volume constant within $\pm 20 \%$.

Westinghouse Hanford generally uses the following two methods to obtain emission samples.

- Record Sampler. A stack record sampler collects airborne particles on a filter that is exchanged and evaluated at least weekly for gross alpha and gross beta activity by laboratory analyses. Record samplers provide an indication of amounts and concentrations of radioactive particles being discharged. Record samplers do not have detectors, strip chart records, or alarms.

- Continuous Air Monitor. Continuous air monitors (CAM) serve as warning devices to alert personnel to emission releases that exceed normal operating parameters. A CAM collects particles on a filter monitored continuously by a radiation detector. The CAM filter also may be used as a backup for the record sample if needed.

Some stacks also emit radionuclide gases and volatiles not collectible on the particulate filters. These gases and volatiles are sampled as follows.

- Iodine and volatile forms of antimony, ruthenium, and tin, if present in an emission, are collected on silver-zeolite or charcoal cartridges used to sample emissions of selected stacks at the Plutonium-Uranium Extraction (PUREX) Plant, Tank Farms, and an evaporator in the 200 Areas and at facilities in the 300 and 400 Areas.

- Tritium $\left({ }^{\beta} \mathrm{H}\right)$ is collected on silica-gel columns used to sample emissions from the Fast Flux Test Facility (FFTF) Combined Exhaust. 
- Plutonium-241, emitted from main stacks at the PUREX Plant and Plutonium Finishing Plant, is calculated from the plant inventories.

- Radon-220, emitted from the PUREX Plant main stack, is calculated from ${ }^{212} \mathrm{~Pb}$ measurements.

- Argon-41 (a noble gas) is sampled from the emissions of the FFTF combined exhaust.

\section{B.2 LIQUID EFFLUENT SAMPLE COLLECTION AND ANALYSIS}

Liquid effluent sample collection schemes are designed to provide a representative sample of the effluent. Volume proportionality and extraction from a well-mixed stream are accomplished where practicable. Liquid effluent streams are sampled using the following: (1) weekly grab sampling, (2) batch sampling from a retention area, (3) incremental time-proportional sampling, (4) incremental flow-proportional sampling, and (5) continuous flow-proportional sampling.

Samples of the liquid effluents are collected for one month, composited, and then analyzed for total alpha and total beta activity, as well as for specific nonradioactive parameters. Specific radionuclide analyses also are performed on monthly composites that contain total alpha or total beta concentrations greater than predetermined limits.

\section{B.3 DATA HANDLING}

Radionuclide air emission and liquid effluent data received are as follows:

- Stack record air sampler flow rates, total flow volume, and sample times

- Stack flow rates based on ventilation measurements or stack flow meters

- Liquid discharge volumes

- Sample analysis data.

These data are reviewed continually for consistency and errors. Concentration values are calculated weekly, monthly, and quarterly and compared with past and present facility releases and with environmental release guides. All anomalies are investigated.

Generally, total alpha and total beta analysis results do not accurately reflect specific radionuclide emissions. However, if alpha results indicate a release concentration much less than the most restrictive limit for specific alpha-emitting radionuclide, the discharge is assumed to be within release limits for all alpha emitters. The same assumption can be made about total beta results that are less than the most restrictive limit for beta emitters. Exceptions to these assumptions exist. In liquids, results for total beta do not include ${ }^{3} \mathrm{H}$, low-energy beta emitters, and volatile alpha and beta emitters that may be driven off with 


\section{WHC-EP-0527-3}

evaporation (e.g., ${ }^{129} \mathrm{I}$ and ${ }^{210} \mathrm{Po}$ ). Radioactive gases and volatile radionuclides are not included in gross analyses of air samples. Separate analyses are performed for ${ }^{3} \mathrm{H}$, gases, and volatiles when appropriate. 


\section{WHC-EP-0527-3}

This page intentionally left blank. 
WHC-EP-0527-3

APPENDIX C

\section{GLOSSARY}


WHC-EP-0527-3

This page intentionally left blank.

C-2 
WHC-EP-0527-3

\section{APPENDIX C}

\section{GLOSSARY}

Air Emission - An airborne discharge released to the environment from a designed system. Emissions of concern are those requiring evidence that contaminant concentrations are within regulatory guidelines.

Background Radiation - Naturally occurring radioactivity in air, water, soil, and manufactured materials; examples are cosmic rays, uranium, radium, thorium, and potassium.

Calibration - Determining the correct value, by measurement or comparison with a standard, of each scale reading on a meter or other device, or correct value for each setting of a control knob.

Crib - An underground structure into which radioactive liquid effluents are discharged for the purpose of most radionuclides being sorbed in the soil above the groundwater.

Derived Concentration Guide (DCG) - Concentration values for radionuclides in air or water that individually would result in an annual maximum effective dose equivalent of $100 \mathrm{mrem} / \mathrm{yr}$ to any member of the public under specific conditions of exposure. Those conditions relate to a set daily amount of radioactively contaminated water ingested or radioactively contaminated air breathed or in which the person is immersed.

Deviation - A departure from normal requirements.

Effective Dose Equivalent (EDE) - The sum of the products of the dose equivalent received by specified tissues of the body and a tissue-specific weighting factor. This sum is a riskequivalent value and can be used to estimate the health-effects risk of the exposed individual. The tissue-specific weighting factor represents the fraction of the total health risk resulting from uniform whole-body irradiation that would be contributed by that particular tissue. The effective dose equivalent includes the committed effective dose equivalent from internal deposition of radionuclides and the effective dose equivalent caused by penetrating radiation from sources external to the body. Effective dose equivalent is expressed in units of rem or sievert.

Exhaust System - Any system that mechanically vents airborne or gaseous material to the atmosphere.

Facility - A processing plant, tank farm, shop, laboratory, powerhouse, or laundry, including all contiguous land, structures, storage, and disposal sites. 
Fission Products - Atoms, usually radioactive, formed by splitting larger atoms such as uranium and plutonium.

Flow Total - The cumulative measurement of the quantity of liquid or air in terms of mass or volume per unit time.

Grab Sample - A single sample removed from an effluent or emission over a short or nearly instantaneous time interval.

Half-Life - The length of time required for a radionuclide to lose one half of its radioactivity.

High-efficiency particulate air (HEPA) filter - To qualify as high efficiency, a filter must achieve a removal efficiency of $99.97 \%$ in the laboratory and $99.95 \%$ after installation for $>3.0 \mathrm{E}-5-\mathrm{cm}(0.3-\mu \mathrm{m})$ particulates.

Isokinetic - A condition that results when the velocity of the stream entering a sampling probe is identical to the velocity of the stream being sampled. (See Near Isokinetic.)

Liquid Effluent - A liquid stream released to the environment from a designed system. Effluents of concern are those requiring evidence that contaminant concentrations are within regulatory guidelines.

Liquid Effluent or Air Emission Monitoring - Measuring contaminants in the discharge stream (liquid or air, respectively) continuously or in near real time.

Liquid Effluent or Air Emission Sampling - Collecting a representative sample (liquid or air, respectively) and performing laboratory analyses.

Mixed Waste - Radioactive waste that also contains hazardous waste constituents.

Monitoring System - Instrumentation that provides the near real-time measurement of an airborne or liquid waste stream parameter. The system includes a detector and associated readout components. A continuous monitoring system measures the stream parameter(s) continuously. A radiation monitoring system is a system in which radiation is the measured parameter. An integrating monitoring system integrates the instantaneously measured parameter over a time period. A sampling system is different from a monitoring system in that it does not measure and read out a near real-time stream parameter.

Near Isokinetic - A condition in which the velocity of a stream entering a sampling probe is within $\pm 20 \%$ of the isokinetic condition.

Noncompliance - A condition resulting when a facility, system, equipment, or procedure does not conform to the requirements. 
Proportional Sampling - The ratio of the volume of the sample taken to the volume of the discharge. This can be done by batch sampling or maintaining a constant sample ratio as in flow-proportional sampling.

Quality Assurance - The method for verifying planning, implementation methods, training, control, and management of all aspects of a program. Documentation provided should be adequate to permit a knowledgeable person to reconstruct results from information supplied or to obtain comparable results with similar instrumentation and procedures.

Radioactive Solid Waste - Any solid material that contains concentrations of radioactive materials exceeding the surface-soil contamination standards.

Record Sample - A representative sample collected in a sampling system (known as a record sampler) for laboratory analysis and used as the basis for reporting the amount and concentration of material released to the environment.

Representative Sample - When the average stream parameter being measured in the sample is in the same average proportion in the discharge.

Sampling System - Instrumentation and equipment that removes part of a liquid or airborne waste stream for subsequent quantitative determination of a stream parameter. The system generally uses such devices as filters, other sample collection media, or effluent traps. A continuous sampling system removes a part of the stream continuously, except during sample change, maintenance, or other necessary outages. An incremental sampling system removes a sample of a discharge stream when either a preset amount of time elapses or an established sample volume of discharge has been collected. Incremental sampling systems normally are capable of sampling only once or repetitively. The increments are designed to be short enough to provide a representative sample. A grab sampling system removes part of the stream at one, nearly instantaneous time or part of the stream over a period of time. 
WHC-EP-0527-3

This page intentionally left blank. 


\section{DISTRIBUTION}

Number of copies

QFFSITE

2

U.S. Department of Energy-Headquarters

G. Chou

EM-322

K. C. Duvall

EH-232

U.S. Environmental Protection Agency

Hanford Project Office

712 Swift Boulevard, Suite 5

Richland, WA 99352

D. R. Sherwood

1

U.S. Environmental Protection Agency, Region 10

1200 Sixth Avenue

Seattle, WA 98087

R. W. Poeten

1

Washington Public Power Supply System

P.O. Box 968, Richland, WA 99352

R. Chitwood

1020

6

Washington State Department of Health

P.O. Box 47827

Olympia, WA 98504-7827

J. Blacklaw

LE-13

A. W. Conklin

LE-13

R. A. Danielson

LE-13

J. Ericksen

LF-13

K. Fox-Williams

LE-13

R. R. Mooney

LE-13

Distr-1 
WHC-EP-0527-3

\section{DISTRIBUTION (continued)}

QNSITE

27

U.S. Department of Energy-

Richland Operations Office

G. M. Bell

E. A. Bracken

A5-52

C. E. Clark

A5-19

S. S. Clark

A5-15

E. B. Dagan

R3-81

M. Dev

A5-15

P.F.X. Dunigan Jr.

S7-53

D. T. Evans

A5-15

M. J. Furman

A5-55

R. E. Gerton

R3-80

J. M. Hennig (4)

S7-54

R. G. Holt

S7-55

R. A. Holten

A5-15

J. R. Hunter

A5-55

R. D. Izatt

R3-81

J. E. Mecca (3)

A7-50

R. O. Puthoff, Jr.

R3-81

J. E. Rasmussen

A5-10

S. D. Stites

A5-15

DOE-RL Reading Room

A5- 15

$\mathrm{H} 2-53$

2

Bechtel Hanford, Inc.

L. A. Mihalik

H4-79

S. R. Weil

H4-79

Pacific Northwest Laboratory

W. J. Bjorklund

P7-79

T. D. Chikalla

P7-75

R. L. Dirkes

K6-61

G. W. Gee

K6-77

R. W. Hanf, Jr.

K6-61

A. K. Ikenberry

P7-68

R. E. Jaquish

B1-34

D. L. Klages

S2-34

G. V. Last

K6-84 


\section{DISTRIBUTION (continued)}

Pacific Northwest Laboratory (continued)

T. M. Poston K6-61

K. Rhoads K3-54

R. G. Schreckhise H2-52

J. K. Soldat K3-54

R. M. Smith K6-96

W. D. Webber K6-96

R. K. Woodruff (5) P7-68

PNL Technical Files $\quad$ K1-11

Westinghouse Hanford Company

M. R. Adams

H6-30

S. E. Albin

T1-06

D. Alison

R1-52

J. A. Ard

T7-37

H. Babad

57-30

J. M. Barnett

T1-30

D. L. Barron

S2-12

W. B. Barton

LA-75

J. A. Bates

H6-22

M. L. Bell

T6-16

D. D. Beers

T7-37

L. D. Berneski

L6-04

B. E. Bielicki

H5-72

D. L. Bjorklund

S6-01

R. J. Bliss

B3-50

D. M. Bogen

S6-65

R. J. Boom

T1-30

G. F. Boothe

G6-46

L. E. Borneman

R2-06

F. E. Boyd

S6-12

S. L. Brey

T6-12

J. R. Brockman

G6-57

M. J. Brown

L6-55

S. A. Burris

H6-25

R. A. Carlson

H6-03

G. D. Carpenter

H6-30

F. M. Coony

H5-33

S. N. Cory

H6-20

G. M. Crummel

R1-51 


\section{DISTRIBUTION (continued)}

Westinghouse Hanford Company (continued)

J. R. Culmer

$\mathrm{X} 0-21$

B. L. Curn

W. E. Davis

T1-30

H. L. Debban

H6-20

C. DeFigh-Price

L6-25

L. P. Diediker (100)

S7-30

L. D. Dillon

T1-30

D. A. Dodd

J. J. Dorian

H6-29

T6-50

H6-20

R. M. Doyle

S5-11

G. L. Dunford

R2-50

G. E. Entrop

B. G. Erlandson

S6-70

D. L. Flyckt

H6-20

T7-38

R. D. Fox

H6-07

T. P. Frazier

T1-30

J. C. Fulton

$\mathrm{X} 0-17$

R. D. Gaines

T5-57

C. J. Geier

R2-36

D. A. Gilles

S2-14

B. P. Gleckler

T1-30

S. D. Godfrey

C. C. Gosney

B2-35

$\mathrm{X} 9-05$

E. M. Greager

H6-20

W. T. Gretsinger

R. D. Gustavson

R1-52

R1-51

K. A. Hadley

R3-56

D. L. Hagel

H5-33

R. D. Haggard

H6-20

M. J. Hall

T6-07

W. H. Hamilton, Jr.

N3-10

D. G. Hamrick

S6-15

R. E. Heineman, Jr.

R3-12

G. C. Henckel III

H6-04

D. R. Herman

S2-12

M. E. Hevland

R3-12

N. A. Homan

H6-02

G. S. Hunacek

$\mathrm{X} 0-41$

D. B. Jensen

H6-20

A. R. Johnson

$\mathrm{H} 6-20$ 


\section{DISTRIBUTION (continued)}

Westinghouse Hanford Company (continued)

D. L. Johnson

S6-17

R. E. Johnson

T1-30

S. R. Johnson

T7-37

T. F. Johnson

$\mathrm{X} 0-21$

V. G. Johnson

H6-06

F. M. Jungfleisch

$\mathrm{T} 7-38$

J. J. Kapadia

$\mathrm{X} 0-21$

M. Kaviani

$\mathrm{X} 3-60$

J. R. Knight

B3-04

A. G. Law, Sr.

H6-06

R. E. Lerch

B3-02

D. W. Lindsey

L6-04

G. A. Lovejoy

B1-32

R. P. Marshall, Jr.

T6-14

P. J. Martell

T1-30

D. J. McBride

T5-54

D. C. McCann

B1-18

M. J. McCarthy

L6-79

H. E. McGuire

B3-63

S. M. McKinney

T1-30

J. C. Midgett

N2-51

T. D. Miller

D. L. Mitchell

R2-77

T. E. Morris

H6-32

L6-25

K. J. Moss

$\mathrm{X} 0-21$

J. R. Murkowski

H6-26

L. A. Nelsen

N2-50

J. M. Nickels

H6-22

R. R. Nielson

T6-12

S. T. Noga

S6-17

S. H. Norton

T3-01

W. L. Osborne

T7-05

M. A. Payne

57-14

C. J. Perkins

$\mathrm{X} 0-21$

K. A. Peterson

H6-22

R. D. Pierce

T3-04

R. K. P'Pool

T1-30

J. R. Prilucik

T6-24

D. G. Ranade

B2-16

L. W. Roberts

L6-05

Distr-5 


\section{DISTRIBUTION (continued)}

Westinghouse Hanford Company (continued)

F. A. Ruck III

H6-23

J. P. Schmidt

$\mathrm{X} 3-73$

J. W. Schmidt

H6-20

M. J. Silvia

H6-25

D. R. Speer

S6-65

L. L. St. Georges

H6-20

D. J. Sommer

H5-49

L. Synoground

T4-03

K. M. Tamburello

T1-30

S. P. Thomas (5)

T1-30

R. J. Thompson

S6-19

R. R. Thompson

H6-32

W. E. Toebe

H6-22

G. L. Troyer

T6-50

D. A. Turner

S7-15

L. W. Vance

H4-16

E. P. Vodney

B3-50

R. E. Vogel

T6-20

E. C. Vogt

T5-50

J. L. Waite

B2-35

H. L. Wedlick

B5-23

D. L. Wegener

R1-62

V. R. Weil

G3-10

R. D. Weissenfels

S6-70

T. M. Wintczak

H6-27

C. D. Wittreich

H6-03

R. D. Wojtasek

S7-84

C. D. Wollam

S6-17

D. E. Wood

H0-33

M. T. York

H6-32

K. L. Young

S3-30

E. Yusis

G7-14

J. P. Zoric

$\mathrm{X} 0-21$

Central Files

L8-04

Correspondence Control

A3-01

Information Release Services (3)

A3-36

President's Office

B3-01

Publication Services

R1-08

EPIC

H6-08 